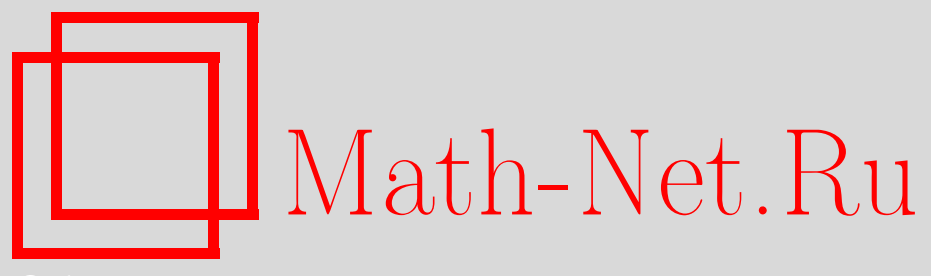

Ф. Радулеску, Эндоморфизмы пространств виртуальных векторов, неподвижных под действием дискретной группы, УМН, 2016, том 71, выпуск 2, 121-178

DOI: https://doi.org/10.4213/rm9713

Использование Общероссийского математического портала Math-Net.Ru подразумевает, что вы прочитали и согласны с пользовательским соглашением http: //www. mathnet.ru/rus/agreement

Параметры загрузки:

IP : 54.157 .27 .8

26 апреля 2023 г., 12:11:34

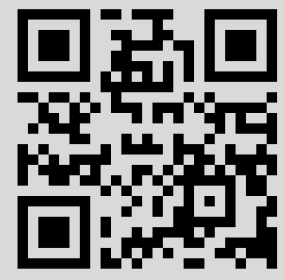




\title{
Эндоморфизмы пространств виртуальных векторов, неподвижных под действием дискретной группы
}

\author{
Ф. Радулеску
}

Рассматривается унитарное представление $\pi$ дискретной группы $G$, которое, будучи ограничено на почти нормальную подгруппу $\Gamma \subseteq G$, является представлением типа II. Изучается ассоциированное унитарное представление $\bar{\pi}^{\mathrm{p}}$ группы $G$ на гильбертовом пространстве "виртуальных" $\Gamma_{0}$-инвариантных векторов, где $\Gamma_{0}$ пробегает подходящий класс подгрупп конечного индекса группы $Г$. Унитарное представление $\bar{\pi}^{\mathrm{P}}$ группы $G$ однозначно определяется требованием, что операторы Гекке для всех $\Gamma_{0}$ являются "клеточно-матричными коэффициентами" представления $\bar{\pi}^{\mathrm{p}}$.

Если $\left.\pi\right|_{\Gamma}$ - целое кратное регулярного представления, то существует подпространство $L$ гильбертова пространства представления $\pi$, играющее роль фундаментальной области для Г. В этом случае пространство Г-инвариантных векторов отождествляется с $L$. Когда $\left.\pi\right|_{\Gamma}$ не является целым кратным регулярного представления (например, если $G=$ $\operatorname{PGL}(2, \mathbb{Z}[1 / p]), \Gamma$ - модулярная группа, $\pi$ принадлежит дискретной серии представлений группы $\operatorname{PSL}(2, \mathbb{R})$, а Г-инвариантные векторы являются каспидальными формами), мы считаем, что $\pi$ есть ограничение на подпространство $H_{0}$ большего унитарного представления, имеющего подпространство $L$ как выше.

Операторный угол между проекцией $P_{L}$ на $L$ (обычно являющейся характеристической функцией фундаментальной области) и проекцией $P_{0}$ на подпространство $H_{0}$ (обычно являющейся проекцией Бергмана на пространство аналитических функций) служит аналогом пространства Г-инвариантных векторов.

Доказано, что характер унитарного представления $\bar{\pi}^{\mathrm{p}}$ однозначно определяется характером представления $\pi$.

Библиография: 53 названия.

Ключевые слова: унитарные представления, операторы Гекке, формулы следа.

DOI: $10.4213 / \mathrm{rm} 9713$

Работа выполнена при поддержке фонда PRIN-MIUR и Румынского национального агентства научных исследований (проект PN-II-ID-PCE-2012-4-0201). 


\section{СоДЕРЖАНИЕ}

1. Введение и основные результаты . . . . . . . . . . . . . . . . . . 122

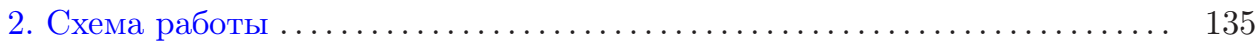

3. Аксиомы для построения гильбертовых пространств Г-инвариантных векторов .......................................... 142

4. Примеры представлений и ассоциированных пространств Г-инвариантных векторов ........................... 153

5. Построение представления $\bar{\pi}_{0}^{\mathrm{p}}$ при отсутствии Г-блуждающего порож-

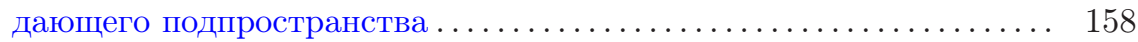

6. Значения характера $\theta_{\pi_{0}}$, ассоциированного с представлением $\pi_{0} \ldots \ldots, 166$

7. Случай, когда представление $\pi$ допускает “квадратный корень" $\pi_{0} \otimes \pi_{0}^{\text {op }} 168$

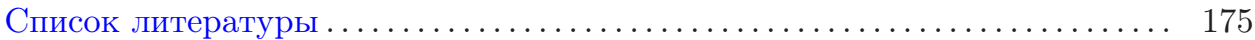

\section{1. Введение и основные результаты}

Пусть $G$ - счетная дискретная группа, Г - почти нормальная подгруппа и $\mathscr{S}$ - минимальная решетка пересечений подгрупп конечного индекса группы Г, замкнутая относительно операции

$$
\Gamma_{0} \rightarrow\left(\Gamma_{0}\right)_{\sigma}=\Gamma_{0} \cap \sigma \Gamma_{0} \sigma^{-1}, \quad \sigma \in G .
$$

Всюду в этой работе предполагается, что пересечение подгрупп из $\mathscr{S}$ есть единичный элемент.

Рассмотрим унитарное (проективное) представление $\pi$ группы $G$ в гильбертовом пространстве $H$. Везде в этой работе мы предполагаем, что $\left.\pi\right|_{\Gamma}$ есть кратное левого регулярного представления $\lambda_{\Gamma}$ (см. [50], [44]) группы $Г$ (возможно, скрещенное с коциклом, если $\pi$ проективно). При этом условии мы строим гильбертовы пространства $H^{\Gamma}$ Г-инвариантных векторов. Более общо, мы строим гильбертовы пространства $H^{\Gamma_{0}} \quad \Gamma_{0}$-инвариантных векторов, когда $\Gamma_{0}$ пробегает $\mathscr{S}$.

Мы называем вышеупомянутые векторы для $\Gamma_{0} \in \mathscr{S}$ "виртуальными" $\Gamma_{0}$-инвариантными векторами, так как они обычно соответствуют $\Gamma_{0}$-инвариантным плотно определенным линейным функционалам на $H$. Гильбертовы пространства $H^{\Gamma_{0}}, \Gamma_{0} \in \mathscr{S}$, не являются истинными подпространствами в $H$; они расположены в расширении - в смысле троек Гельфанда (см. [18]) - заданного гильбертова пространства (см. определение 14). Для всех подгрупп $\Gamma_{0}, \Gamma_{1} \in \mathscr{S}$ таких, что $\Gamma_{1} \subseteq \Gamma_{0}$, существует каноническое изометрическое вложение $H^{\Gamma_{0}} \subseteq H^{\Gamma_{1}}$.

Проиллюстрируем это в случае левого регулярного представления $\lambda_{\Gamma}$ группы $\Gamma$ в унитарную группу $\mathscr{U}\left(l^{2}(\Gamma)\right)$ гильбертова пространства $l^{2}(\Gamma)$. В этом случае пространство Г-инвариантных векторов есть одномерное пространство, порожденное постоянными функциями на Г. Это пространство можно рассматривать как пространство плотно определенных линейных функционалов на $l^{2}(\Gamma)$. В общем случае в этом формализме $l^{2}(\Gamma)^{\Gamma_{0}}=\ell^{2}\left(\Gamma_{0} \backslash \Gamma\right)$. 
В общем случае, если унитарное представление $\pi$ в гильбертово пространство $H$ является целым кратным левого регулярного представления $\lambda_{\Gamma}$ дискретной группы $\Gamma$, то существует подпространство $L$ такое, что Г-эквивариантным образом выполняется

$$
H \cong l^{2}(\Gamma) \otimes L,\left.\quad \pi\right|_{\Gamma} \cong \lambda_{\Gamma} \otimes \operatorname{Id}_{L}
$$

В этом случае также очевидно, что можно отождествить пространство Г-инвариантных векторов с гильбертовым пространством $L$. При этом отождествлении $L$ уже не является истинным подпространством в $H$. Более того, $L$ не единственно.

Если унитарное представление $\pi$ не является целым кратным левого регулярного представления $\lambda_{\Gamma}$, то мы будем использовать подпространство $L$ из большего представления группы $G$, которое содержит данное представление $\pi$ как подпредставление. В этом случае мы докажем ниже, что операторный угол между проекцией на подпространство подпредставления и проекцией на $L$ может быть использован для построения пространства Г-инвариантных векторов и его скалярного произведения.

Это аналогично формуле скалярного произведения Петерссона для автоморфных форм, приведенной в [34], где для того, чтобы ввести скалярное произведение на автоморфных формах, которые являются "виртуальными" $\operatorname{PSL}(2, \mathbb{Z})$-инвариантными векторами для ограничения на $\operatorname{PSL}(2, \mathbb{Z})$ представлений $\pi_{n}, n \geqslant 2$, из дискретной серии группы $\operatorname{PSL}(2, \mathbb{R})$, была использована "измеряющая шкала", состоящая из фундаментальной области $F$ для действия группы $\operatorname{PSL}(2, \mathbb{Z})$ на верхней полуплоскости. Большее унитарное представление, содержащее представление $\pi_{n}$ в качестве подпредставления, получается следующим образом: если $\pi_{n}$ реализовано как унитарное представление на пространстве $H^{2}\left(\mathbb{H}, \nu_{n}\right)$, состоящем из интегрируемых с квадратом аналитических функций относительно меры $\nu_{n}$ на $\mathbb{H}$, то большее унитарное представление, содержащее $\pi_{n}$ в качестве подпредставления, реализуется на соответствующем гильбертовом пространстве $L^{2}\left(\mathbb{H}, \nu_{n}\right)$. Пространство суммируемых с квадратом функций с носителями в $F$ играет роль подпространства $L$ как выше.

Пусть $\sigma$ - групповой элемент из $G$. Мы используем обозначения, введенные перед формулой (1). Очевидно, что $\sigma \Gamma_{\sigma^{-1}} \sigma^{-1}=\Gamma_{\sigma}$. Из этого следует, что представление $\pi$ индуцирует унитарное преобразование (обозначаемое $\bar{\pi}^{\mathrm{p}}(\sigma)$ ), которое отображает $H^{\Gamma_{\sigma-1}}$ на $H^{\Gamma_{\sigma}}$.

Основная проблема, которую мы рассматриваем в этой работе, - изучение унитарного представления $\bar{\pi}^{\mathrm{p}}$, индуцированного унитарным представлением $\pi$ на гильбертовом пространстве $\bar{H}^{\mathrm{P}}$, полученном путем взятия индуктивного предела гильбертовых пространств $H^{\Gamma_{0}}, \Gamma_{0} \in \mathscr{S}$. Содержание классической проблемы Рамануджана-Петерссона [24], [47], [6] преобразуется в проблему гармонического анализа о слабом унитарном включении представления $\bar{\pi}^{\mathrm{P}}$ в унитарное представление, полученное ограничением на $G$ левого регулярного представления пополнения Шлихтинга [48], [26] группы $G$ относительно подгрупп из семейства $\mathscr{S}$. 
Представление $\bar{\pi}^{\mathrm{p}}$ определяется ассоциированными операторами Гекке [23], [24], [46] на всех уровнях $\Gamma_{0}$. Операторы Гекке являются "клеточно-матричными коэффициентами" ассоциированного унитарного представления $\bar{\pi}^{\mathrm{p}}$. Обозначение $\bar{\pi}^{\mathrm{p}}$ для представления на пространстве всех "виртуальных" $\Gamma_{0}$-инвариантных векторов, $\Gamma_{0} \in \mathscr{S}$, мы используем, чтобы подчеркнуть, что это представление построено с помощью процедуры проконечного пополнения. Когда исходное представление $\pi$ является проективным представлением с коциклом $\epsilon$, а коцикл $\epsilon$ допускает продолжение на пополнение Шлихтинга $\bar{G}$, введенное ниже, построение в этой работе дословно так же работает и в проективном случае.

Представление $\bar{\pi}^{\mathrm{p}}$ в несколько иной форме широко используется в литературе (см., например, [6], [13], [21]). В этой работе мы используем построение $\bar{\pi}$ р в терминах операторных алгебр. Это построение дает возможность получить унитарно эквивалентные формы $\bar{\pi}^{\mathrm{p}}$, которые больше подходят для вычисления следов.

Основной результат настоящей работы - это соответствие между двумя представлениями $\pi$ и $\bar{\pi}^{\mathrm{p}}$. Представление $\bar{\pi}^{\mathrm{p}}$ имеет каноническую клеточно-матричную структуру, связанную с заданным выбором представителей смежных классов для подгрупп из $\mathscr{S}$. Следовательно, мы представляем ассоциированные операторы Гекке как клеточные матрицы, элементы которых являются “локализованными суммами" по смежным классам значений исходного представления $\pi$, ограниченного на пространство $L$, введенное выше (которое является аналогом фундаментальной области для действия группы Г). В качестве следствия мы получаем точную формулу, связывающую характеры $\pi$ и $\bar{\pi}$ p.

Чтобы установить связь между этими двумя представлениями, мы вводим условие суммируемости, которое обеспечивает сходимость сумм для элементов матриц в представлениях, которые мы получаем для операторов Гекке.

ОПРЕДЕЛЕНИЕ 1 . Пусть $\pi_{0}$ - унитарное представление группы $G$ такое, что $\left.\pi\right|_{\Gamma}$ является (не обязательно целым) кратным левого регулярного представления $\lambda_{\Gamma}$. Мы предполагаем, что существует унитарное представление $\pi$ группы $G$ в унитарную группу гильбертова пространства $H$, содержащего $H_{0}$, обладающее следующими свойствами.

(i) Представление $\left.\pi\right|_{\Gamma}$ является целым кратным левого регулярного представления $\lambda_{\Gamma}$. Следовательно, существует гильбертово подпространство $L$ пространства $H$ такое, что

$$
H \cong l^{2}(\Gamma) \otimes L,\left.\quad \pi\right|_{\Gamma} \cong \lambda_{\Gamma} \otimes \operatorname{Id}_{L}
$$

(ii) Пусть $e$ - единичный элемент группы $G$. Обозначим ортогональную проекцию из $H$ на $L \cong \mathbb{C} e \otimes L$ через $P_{L}$. Мы считаем, что проекции из семейства $\left\{\pi(g) P_{L} \pi\left(g^{-1}\right) \mid g \in G\right\}$ образуют коммутативное семейство.

(iii) Предположим, что $\pi_{0}$ - подпредставление представления $\pi$. Тогда $H_{0} \subseteq H$ является $\pi(G)$-инвариантным и $\pi_{0}$ есть ограничение представления $\pi$ на $H_{0}$. Обозначим через $P_{0}$ ортогональную проекцию из $H$ на $H_{0}$. Ясно, что в этом случае имеем $\pi_{0}(g)=P_{0} \pi(g) P_{0}, g \in G$.

(iv) Произведение операторов $P_{0}$ и $P_{L}$ является оператором со следом. 
(v) Для каждых $g \in G$ и $\Gamma_{0} \in \mathscr{S}$ сумма по смежному классу $\Gamma_{0} g$

$$
\sum_{\theta \in \Gamma_{0} g} P_{L} \pi_{0}(\theta) P_{L}
$$

сходится в пространстве операторов Гильберта-Шмидта $\mathscr{C}_{2}(L)$.

(vi) Сумма следов операторов в сумме (2) сходится абсолютно. Сумма (2) является оператором со следом, и его след равен сумме следов операторов в сумме.

(vii) Размерность Мюррея-фон Неймана $\operatorname{dim}_{\left\{\pi_{0}(\Gamma)\right\}^{\prime \prime}} H_{0}$ (см. [50], [44], [25]) является конечным строго положительным числом.

Условие (vii) можно интерпретировать как то, что $H_{0}$ является конечно порожденным левым гильбертовым модулем над представлением $\left.\pi_{0}\right|_{\Gamma}$, которое является кратным левого регулярного представления группы $Г$.

В основных примерах, рассматриваемых в этой работе, где представления $\pi_{0}$ получаются из унитарных представлений из дискретной серии (проективных) унитарных представлений группы $\operatorname{PSL}(2, \mathbb{R})$ ограничением на $G=\operatorname{PGL}(2, \mathbb{Z}[1 / p])$, условия (iii), (iv) следуют из вычислений в [53]. Условие (ii) выполняется автоматически, если представление $\pi$ получено из унитарного представления Купмана. Действительно, в этом случае проекция $P_{L}$ является оператором умножения на характеристическую функцию фундаментальной области для Г. Кроме того, проекции из этого семейства являются операторами умножения на характеристические функции сдвигов фундаментальной области элементами из $G$.

Вышеупомянутое унитарное представление $\pi_{0}$ группы $G$ каноническим образом определяет унитарное представление $\bar{\pi}_{0}^{\mathrm{p}}$ пополнения Шлихтинга [48] $\bar{G}$ группы $G$ относительно подгрупп из семейства $\mathscr{S}$. Унитарное представление $\bar{\pi}_{0}^{\mathrm{p}}$ действует на $\bar{H}^{\mathrm{p}}$, гильбертовом пространстве пополнения индуктивного предела гильбертовых пространств $H^{\Gamma_{0}}, \Gamma_{0} \in \mathscr{S}$. Эта конструкция строго описывается в теореме 33. Следующий результат дает явное описание клеточноматричных коэффициентов представления $\bar{\pi}_{0}^{\mathrm{p}}$. Это операторы Гекке, ассоциированные с исходным унитарным представлением $\pi_{0}$, которые соответствуют подгруппам $\Gamma_{0} \in \mathscr{S}$. Это представление используется для того, чтобы вычислить характер представления $\bar{\pi}_{0}^{\mathrm{p}}$. В следующей теореме мы используем обозначение $\left(\Gamma_{0}\right)_{\sigma}=\sigma \Gamma_{0} \sigma^{-1} \cap \Gamma_{0}, \Gamma_{0} \in \mathscr{S}, \sigma \in G$.

ТеОрема 2. Пусть $\pi_{0}$ - унитарное представление группы $G$ в унитарную группу $\mathscr{U}\left(H_{0}\right)$ гильбертова пространства $H_{0}$. Предположим, что $\pi_{0}$ удовлетворяет техническим условиям (i)-(vii) из определения 1. Пусть $\bar{\pi}_{0}^{\mathrm{p}}-$ унитарное представление группы $\bar{G}$, введенное выше.

Для каждого $\Gamma_{0} \in \mathscr{S}$ зафиксируем семейство представителей смежных $\left[\Gamma: \Gamma_{0}\right]$

классов для $\Gamma_{0}$ в $\Gamma$ такое, что $\Gamma=\bigcup_{i=1} \Gamma_{0} s_{i}$. Представим $B\left(l^{2}\left(\Gamma_{0} \backslash \Gamma\right)\right)$ матричной единищей

$$
\left(e_{\Gamma_{0} s_{i}}, e_{\Gamma_{0} s_{j}}\right)_{i, j=1,2, \ldots,\left[\Gamma: \Gamma_{0}\right]} \cdot
$$

Для $\Gamma_{0} \in \mathscr{S}$ u $\sigma \in G$ операторы Гекке, ассоииированные с $\pi_{0}$, которые соответствуют двойному смежному классу $\Gamma_{0} \sigma \Gamma_{0}$, таковы:

$$
\left[\Gamma_{0}:\left(\Gamma_{0}\right)_{\sigma}\right] P_{H_{0}^{\Gamma_{0}}} \bar{\pi}_{0}^{\mathrm{p}}(\sigma) P_{H_{0}^{\Gamma_{0}}} .
$$


В силу унитарной эквивалентности

$$
B\left(l^{2}\left(\Gamma_{0} \backslash \Gamma\right)\right) \otimes B(L) \cong B\left(H^{\Gamma_{0}}\right)
$$

и сделанного выше выбора матричной единицы, операторы Гекке в формуле (3) унитарно эквивалентны операторам из $B\left(l^{2}\left(\Gamma_{0} \backslash \Gamma\right)\right) \otimes B(L)$, заданным формулой

$$
\sum_{i, j} \sum_{\theta \in s_{i}^{-1} \Gamma_{0} \sigma \Gamma_{0} s_{j}} P_{L} \pi_{0}(\theta) P_{L} \otimes e_{\Gamma_{0} s_{i}, \Gamma_{0} s_{j}} .
$$

Формула (4) позволяет немедленно сделать следующий вывод.

СледствиЕ 3. Для $\sigma \in G u \Gamma_{0} \in \mathscr{S}$ след оператора Гекке, введенного в (3), вычисляется по формуле

$$
\operatorname{Tr}\left[\left[\Gamma_{0}:\left(\Gamma_{0}\right)_{\sigma}\right] P_{H_{0}^{\Gamma_{0}}} \bar{\pi}_{0}^{\mathrm{p}}(\sigma) P_{H_{0}^{\Gamma_{0}}}\right]=\sum_{s_{i}} \sum_{\theta \in s_{i} \Gamma_{0} \sigma \Gamma_{0} s_{i}^{-1}} \operatorname{Tr}\left(P_{L} \pi_{0}(\theta) P_{L}\right) .
$$

В частном случае $\Gamma_{0}=\Gamma$ формула (4) дает следующее.

Предположим, что $\operatorname{dim}_{\left\{\pi_{0}(\Gamma)\right\} "} H_{0}$ конечна. Пространство $H_{0}^{\Gamma}$ "виртуальных" векторов, инвариантных относительно унитарного представления $\left.\pi\right|_{\Gamma}$, действующего на $H_{0}$, унитарно эквивалентно образу проекции

$$
\mathscr{P}_{\Gamma, L}=\sum_{\gamma \in \Gamma} P_{L} \pi_{0}(\gamma) P_{L} \in B(L),
$$

который является конечномерным подпространством из $L$. Та же унитарная эквивалентность преобразует оператор Гекке, соответствующий смежному классу Г $\sigma$ и действующий на "виртуальных" Г-инвариантных операторах, в оператор

$$
\sum_{\gamma \in \Gamma \sigma \Gamma} P_{L} \pi_{0}(\gamma) P_{L} \in B(L), \quad \sigma \in G .
$$

Сходимость в вышеприведенных формулах следует из технического предположения (v), введенного в определении 1.

В качестве следствия равенства (5) получаем формулу для характера [22], [45], [12], [17] представления $\bar{\pi}_{0}^{\mathrm{p}}$. Это доказывает, что характер представления $\bar{\pi}_{0}^{\mathrm{p}}$ определяется положительно определенной функцией $\phi_{0}$ на $G$, заданной как

$$
\phi_{0}(g)=\operatorname{Tr}\left(P_{L} \pi_{0}(g) P_{L}\right), \quad g \in G .
$$

Мы используем обозначения и определения из формулировки теоремы 2. Мы считаем, что характер $\theta_{\bar{\pi}_{0}^{\mathrm{p}}}$ представления $\bar{\pi}_{0}^{\mathrm{p}}$ группы $\bar{G}$ локально интегрируем относительно меры Хаара на $\bar{G}$. Напомним, что в силу [12] и формулы (13) (см. также другое доказательство в лемме 36) имеем

$$
\theta_{\bar{\pi}_{0}^{\mathrm{p}}}(\sigma)=\lim _{\Gamma_{0} \downarrow e, \Gamma_{0} \in \mathscr{S}} \operatorname{Tr}\left(P_{H_{0}^{\Gamma_{0}}} \bar{\pi}_{0}^{\mathrm{p}}(\sigma) P_{H_{0}^{\Gamma_{0}}}\right) .
$$

Для каждого элемента $g \in G$ обозначим через $\Gamma_{g}^{\text {st }}$ его стабилизатор в $\Gamma$ :

$$
\Gamma_{g}^{\mathrm{st}}=\{\gamma \in \Gamma \mid \gamma g=g \gamma\} .
$$

Используя формулы (5) и (7), получаем следующее утверждение. 
СлЕдствиЕ 4. Значение характера $\theta_{\bar{\pi}_{0}^{\mathrm{p}}}$ на элементе $\sigma \in G$ вычисляется по формуле

$$
\theta_{\bar{\pi}_{0}^{\mathrm{p}}}(\sigma)=\lim _{\Gamma_{0} \downarrow e, \Gamma_{0} \in \mathscr{S}} \frac{1}{\left[\Gamma_{0}:\left(\Gamma_{0}\right)_{\sigma}\right]} \sum_{\substack{\left[\Gamma: \Gamma_{0}\right] \\ \bigcup_{i=1} \Gamma_{0} s_{i}}} \sum_{\theta \in s_{i}^{-1} \Gamma_{0} \sigma \Gamma_{0} s_{i}} \operatorname{Tr}\left(P_{L} \pi_{0}(\theta) P_{L}\right) .
$$

Если группа $\Gamma_{g}^{\mathrm{st}}$ тривиальна, то

$$
\theta_{\bar{\pi}_{0}^{\mathrm{p}}}(g)=\left[\lim _{\Gamma_{0} \downarrow e, \Gamma_{0} \in \mathscr{S}} \frac{1}{\left[\Gamma_{0}:\left(\Gamma_{0}\right)_{g}\right]}\right] \sum_{\gamma \in \Gamma} \operatorname{Tr}\left(P_{L} \pi_{0}\left(\gamma g \gamma^{-1}\right)\right), \quad g \in G .
$$

При подходящих условиях член в правой части равенства (9) совпадает с характером унитарного представления $\pi_{0}$. Предположим, что существует унитарное представление $\bar{\pi}_{0}^{R}$, продолжающее $\pi_{0}$ на локально компактную группу $\bar{G}^{R}$, содержащую $G$ в качестве всюду плотной подгруппы. Тогда формула для следа характера представления $\bar{\pi}_{0}$ зависит только от следа характера представления $\bar{\pi}_{0}^{R}$.

Лемма 5. Пусть $\pi_{0}$ - унитарное представление, удовлетворяющее условиям определения 1. Пусть $\bar{G}^{R}$ - бо́льшая локально компактная группа, содержащая $G$ в качестве всюду плотной подгруппь, такая, что $\Gamma$ - решетка в $\bar{G}^{R}$. Предположим, что выполнены следующие условия:

(а) представление $\pi_{0}$ продолжается до унитарного представления $\bar{\pi}_{0}^{R}$ группъ $\bar{G}^{R}$ в унитарную группу пространства $H$; более того, представление $\bar{\pi}_{0}^{R}$ имеет локально интегрируемый характер относительно меры Хаара на $\bar{G}^{R}$, обозначаемый через “ $\operatorname{Tr}\left(\bar{\pi}_{0}^{R}(\cdot)\right) "=\theta_{\bar{\pi}_{0}^{R}} ;$

(b) представление $\pi$ из определения 1 также продолжается до унитарного представления $\bar{\pi}^{R}$ группы $\bar{G}^{R}$, и равенство $\bar{\pi}_{0}^{R}(g)=P_{0} \bar{\pi}^{R} P_{0}$ выполняется для всех $g \in \bar{G}^{R}$;

(с) множество элементов $g \in \bar{G}^{R}$ с нетривиальной группой $\Gamma_{g}^{\mathrm{st}}$ имеет нулевую меру относительно меры Хаара на $\bar{G}^{R}$.

Тогда справедливы следующие утверждения:

(i) для любого элемента $g \in G$ такого, как в условии (с), имеем

$$
\theta_{\bar{\pi}_{0}^{R}}(g)=\sum_{\gamma \in \Gamma} \operatorname{Tr}_{B(L)}\left(P_{L} \pi_{0}\left(\gamma g \gamma^{-1}\right) P_{L}\right)
$$

(ii) характер унитарного представления $\bar{\pi}_{0}$, ограниченный на $G$ (за исключением точек $g \in G$, для которых $\Gamma_{g}^{\mathrm{st}}$ нетривиален), зависит только от характера представления $\bar{\pi}_{0}^{R}$.

ЗАмечание 6. Рассмотрим сумму в формуле (8):

$$
\phi_{\Gamma_{0}}(\sigma)=\sum_{\Gamma \sum_{i=1}^{\left[\Gamma: \Gamma_{0}\right]} \Gamma_{0} s_{i}} \sum_{\theta \in s_{i}^{-1} \Gamma_{0} \sigma \Gamma_{0} s_{i}} \operatorname{Tr}\left(P_{L} \pi_{0}(\theta) P_{L}\right), \quad \sigma \in G .
$$


Когда $\sigma=e$ и $\Gamma_{0}-$ нормальная подгруппа, этот член равен

$$
\left[\Gamma: \Gamma_{0}\right] \sum_{\Gamma=\bigcup_{i=1}^{\left[\Gamma: \Gamma_{0}\right]} \Gamma_{0} s_{i}} \sum_{\theta \in \Gamma_{0}} \operatorname{Tr}\left(P_{L} \pi_{0}(\theta) P_{L}\right)=\left[\Gamma: \Gamma_{0}\right] \sum_{\gamma \in \Gamma_{0}} \operatorname{Tr}\left(P_{L} \pi_{0}(\gamma) P_{L}\right) .
$$

Нормируя и беря предел

$$
\Phi_{\pi_{0}}(\sigma)=\lim _{\Gamma_{0} \downarrow e, \Gamma_{0} \in \mathscr{S}} \frac{\phi_{\Gamma_{0}}(\sigma)}{\operatorname{dim} H^{\Gamma_{0}}}, \quad \sigma \in G,
$$

получаем характер группы $G$ типа, рассмотренного в [33], [16], [9], [8], [52].

Характеры, полученные в следствии 4 , имеют другую природу: они принимают бесконечное значение на единице и могут принимать бесконечное значение на других элементах группы.

Ниже мы проиллюстрируем содержание теоремы 2 и следствия 4 в частном случае, когда Г-инвариантные векторы являются автоморфными формами. Пусть $G=\operatorname{PGL}(2, \mathbb{Z}[1 / p]), p$ - простое число, Г - модулярная группа, а представление $\pi_{0}=\left.\pi_{n}\right|_{G}, n \in \mathbb{N}, n \geqslant 2$, получено ограничением на $G$ (проективного) унитарного представления из аналитической дискретной серии $\left(\pi_{n}\right)_{n \geqslant 2}$ полупростой группы Ли $\operatorname{PSL}(2, \mathbb{R})$. Пусть $F$ - фундаментальная область для действия модулярной группы на верхней полуплоскости. Пусть $d \nu_{0}(z)=(\operatorname{Im} z)^{-2} d \bar{z} d z-$ каноническая мера на $\mathbb{H}$, которая инвариантна под действием группы $\operatorname{PSL}(2, \mathbb{R})$ преобразованиями Мёбиуса. Тогда проекция $P_{L}$, введенная в определении 1 , является оператором $M_{\chi_{F}}$ умножения на характеристическую функцию области $F$ на $H=L^{2}\left(\mathbb{H},(\operatorname{Im} z)^{-2} d \bar{z} d z\right)$. Унитарное представление $\pi$ является унитарным представлением Купмана группы $G$ на $L^{2}\left(\mathbb{H},(\operatorname{Im} z)^{-2} d \bar{z} d z\right)$, соответствующим действию $G$ на $\mathbb{H}$ (см. примеры 28 и 29).

В этом частном случае проекция $P_{0}$ есть проекция Бергмана на гильбертово пространство представления $\pi_{n}$, которое является пространством аналитических функций на $\mathbb{H}$, суммируемых с квадратом относительно меры $d \nu_{n}(z)=$ $(\operatorname{Im} z)^{n-2} d \bar{z} d z$. Более того, техническое условие (v) из определения 1 эквивалентно условию $L^{2}$-сходимости для воспроизводящих ядер Березина [3] операторов из суммы в (4). Это условие выполняется в описанном здесь частном случае в силу вычислений в [53] и [19; §3.3].

Для ограниченного оператора $A$ на гильбертовом пространстве $H_{n}$ обозначим его символ Березина [3] через $\widehat{A}(\bar{z}, \zeta), z, \zeta \in \mathbb{H}$. Из формулы (9) тогда вытекает следующее утверждение.

СледСтвиЕ 7. Пусть $\sigma$ - элемент из $G$ с тривиальной группой $\Gamma_{\sigma}^{\mathrm{st}}$. Тогда

$$
\begin{aligned}
\theta_{\bar{\pi}_{n}^{\mathrm{p}}}(\sigma) & =\left[\lim _{\Gamma_{0} \downarrow e, \Gamma_{0} \in \mathscr{S}} \frac{1}{\left[\Gamma_{0}:\left(\Gamma_{0}\right)_{g}\right]}\right] \int_{\mathbb{H}} \widehat{\pi_{n}(\sigma)}(\bar{z}, z) d \nu_{0}(z) \\
& =\left[\lim _{\Gamma_{0} \downarrow e, \Gamma_{0} \in \mathscr{S}} \frac{1}{\left[\Gamma_{0}:\left(\Gamma_{0}\right)_{\sigma}\right]}\right] \int_{\mathbb{H}} \frac{1}{\sigma z-\bar{z}} d \nu_{0}(z) \\
& =\left[\lim _{\Gamma_{0} \downarrow e, \Gamma_{0} \in \mathscr{S}} \frac{1}{\left[\Gamma_{0}:\left(\Gamma_{0}\right)_{\sigma}\right]}\right] \theta_{\pi_{n}}(\sigma) .
\end{aligned}
$$


Тот факт, что интеграл $\int_{\mathbb{H}} \widehat{\pi_{n}(\sigma)}(\bar{z}, z) d \nu_{0}(z)$ в $(12)$ является характером $\theta_{\pi_{n}}$ представления $\pi_{n}$, доказан в [32] с помощью квантизации Березина. В этом контексте формула для суммы в (9) вычислена другим методом в [53].

В разделе 7 мы применяем конструкцию Г-инвариантных "виртуальных" векторов из теоремы 2 к диагональным представлениям группы $G$ вида $\pi_{0} \otimes \pi_{0}^{\text {op }}$, действующим на гильбертовом пространстве $H_{0}$. Здесь $\pi_{0}^{\mathrm{op}}-$ комплексно сопряженное представление, ассоциированное с представлением $\pi_{0}$. Мы используем унитарно эквивалентное представление гильбертовых пространств, состоящих из Г-инвариантных векторов.

Это представление унитарно эквивалентно унитарному представлению $\mathrm{Ad} \pi_{0}$, действующему на гильбертовом пространстве, состоящем из идеала операторов Гильберта-Шмидта $\mathscr{C}_{2}\left(H_{0}\right) \subseteq B\left(H_{0}\right)$. Пространство $\mathscr{V} \Gamma_{0}$ "виртуальных" $\Gamma_{0}$-инвариантных векторов является алгеброй операторов фон Неймана $X \in$ $B\left(H_{0}\right)$, которые коммутируют с $\pi_{0}\left(\Gamma_{0}\right)$. Эта алгебра есть (см. пример 30) коммутант $\mathscr{A}_{\Gamma_{0}}=\left\{\pi_{0}\left(\Gamma_{0}\right)\right\}^{\prime}$.

Для фактора $M$ типа $\mathrm{II}_{1}$ со следом $\tau$ мы обозначаем через $L^{2}(M, \tau)$ гильбертово пространство, ассоциированное с $\tau$ с помощью конструкции Гельфанда-Наймарка-Сигала [50]. Это гильбертово пространство получается с помощью гильбертова пополнения из скалярного произведения, индуцированного следом на векторном пространстве $M$. По предположению размерность $\operatorname{dim}_{\left\{\pi_{0}\left(\Gamma_{0}\right)\right\}^{\prime \prime}} H_{0}$ конечна, отсюда мы заключаем [50], что $\mathscr{A}_{\Gamma_{0}}$ есть алгебра фон Неймана типа II. Тогда гильбертово пространство $\Gamma_{0}$-инвариантных векторов является $L^{2}$-пространством $L^{2}\left(\mathscr{A}_{\Gamma_{0}}, \tau\right)$, ассоциированным с фактором фон Неймана $\mathscr{A}_{\Gamma_{0}}$ типа $\mathrm{II}_{1}$. Индуктивный предел этих гильбертовых пространств, когда $\Gamma_{0}$ пробегает $\mathscr{S}$, имеет естественную интерпретацию в терминах базисной конструкции Джоунса (пример 30, см. также конструкцию в [39]).

Это особенно интересно, когда $\pi_{0}$ - вышеупомянутое представление $\pi_{n}$ (т. е. $G=\operatorname{PGL}(2, \mathbb{Z}[1 / p]), p$ - простое, $\Gamma$ - модулярная группа, $\pi_{n}$ получено ограничением на $G$ из дискретной серии унитарных представлений группы $\operatorname{PSL}(2, \mathbb{R}))$. Рассмотрим унитарное представление Купмана $\pi_{\text {Коор }}$ (см. пример 28 ), ассоциированное с сохраняющим меру действием группы $G$ на пространстве $\mathbb{H}$, наделенном мерой $\nu_{0}$, введенной выше.

Тогда в силу [42] или теории квантизации Березина ([3], см. также [37]) с точностью до унитарного сопряжения имеем

$$
\pi_{\text {Koop }} \cong \pi_{n} \otimes \pi_{n}^{\text {op }}, \quad n \geqslant 2 .
$$

В силу вышеупомянутой эквивалентности анализ представления группы $G$ на пространствах "виртуальных" $\Gamma_{0}$-инвариантных векторов для представления $\pi_{n} \otimes \pi_{n}^{\text {op }}$ приводит к анализу пространств $\Gamma_{0}$-инвариантных векторов, $\Gamma_{0} \in \mathscr{S}$, для представлений Купмана и соответствующего унитарного действия группы $G$ на этих пространствах. Последнее представление соответствует действию операторов Гекке на формах Маасса [31]. Используя этот метод, мы получаем в разделе 7 (см. также [37]) конкретные алгебраические формулы, связывающие матричные коэффициенты представления $\pi_{n}$ с выражением для операторов Гекке, ассоциированных с $\pi_{\text {Коор }}$. 
Этот метод полезен для понимания унитарного представления Купмана $\pi_{\text {Коор }}$, из которого выводится действие операторов Гекке на формах Маасса. Мы используем естественный “квадратный корень" из представления $\pi_{\text {Коор }}$, задаваемый представлением из дискретной серии, как в формуле (13). Представление $\pi_{n} \otimes \pi_{n}^{\text {op }}$ лучше поддается обработке, так как с помощью интерпретации в терминах операторных алгебр канонически определяются гильбертовы пространства "виртуальных" $\Gamma_{0}$-инвариантных векторов, $\Gamma_{0} \in \mathscr{S}$. Мы поясняем эту конструкцию ниже.

Пусть $\mathscr{R}(G)$ - алгебра фон Неймана, порожденная операторами правой свертки $\rho(g), g \in G$, действующими на $\ell^{2}(G)$. Это коммутант алгебры левых сверток $\mathscr{L}(G)$, порожденной операторами левой свертки $\lambda_{g}, g \in G$, действующими на том же гильбертовом пространстве.

Рассмотрим следующие алгебры, связанные с включениями $\Gamma \subseteq G, K \subseteq \bar{G}$. Пусть $\mathscr{H}_{0}(K, \bar{G})=\mathbb{C}(K \backslash \bar{G} / K)$ - алгебра двойных смежных классов подгруппы $K$ в $\bar{G}$ (см. [1], [28]). Обозначим алгебру Гекке $\mathbb{C}(\Gamma \backslash G / \Gamma)$, порожденную двойными смежными классами подгруппы $\Gamma$ в $G$, через $\mathscr{H}_{0}(G, \Gamma)$. Последняя алгебра изоморфна алгебре Гекке $\mathscr{H}_{0}(K, \bar{G})$. Алгебра Гекке обладает естественной операцией инволюции, которая индуцирует структуру *-алгебры.

Алгебра Гекке обладает каноническим точным *-представлением (см. [7]) в $B\left(\ell^{2}(\Gamma \backslash G)\right)$. Обозначим элементы стандартного базиса пространства $\ell^{2}(\Gamma \backslash G)$ через $[\Gamma \sigma], \sigma \in G$. Наложенное нами условие равенства индексов $\left[\Gamma: \Gamma_{\sigma}\right]=$ $\left[\Gamma: \Gamma_{\sigma^{-1}}\right], \sigma \in G$, означает, что состояние $\langle\cdot[\Gamma],[\Gamma]\rangle$, которое определено на $B\left(\ell^{2}(\Gamma \backslash G)\right)$, становится следом при ограничении на образ алгебры Гекке. Приведенная $C^{*}$-алгебра алгебры Гекке является замыканием по норме в представлении в $B\left(\ell^{2}(\Gamma \backslash G)\right)$ алгебры Гекке $\mathscr{H}_{0}(G, \Gamma)$. Она обозначается через $\mathscr{H}_{\text {red }}(G, \Gamma)$. Ясно, что $\mathscr{H}_{0}(G, \Gamma) \cong \mathscr{H}_{0}(K, \bar{G})$.

$C^{*}$-алгебра $C^{*}(\bar{G})$ содержит (вводимую в определении 8 ниже) каноническую систему операторов [35], ассоциированную с максимальной компактной подгруппой $K$. Мы поясним ниже, что *-представления этой системы операторов, указанные в формуле (18) леммы 9, содержат всю информацию о представлении $\pi_{0}$. Эти морфизмы являются основными строительными блоками представления алгебр Гекке, ассоциированного с представлением вида $\pi_{0} \otimes \pi_{0}^{\text {op }}$ (см. теоремы 10 и 12). Главным инструментом для изучения унитарных представ-

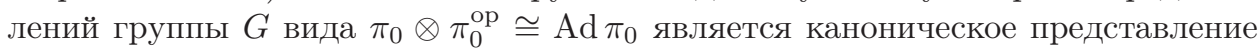
алгебры Гекке $\mathscr{H}_{0}(G, \Gamma)$ в $\mathscr{R}(G) \otimes B(L)$, которое описывается ниже.

ОПРЕДЕЛЕниЕ 8 . Пусть $L(K, \bar{G})=\mathbb{C}\left(\chi_{\sigma K} \mid \sigma \in G\right)$ - линейное подпространство пространства $C^{*}(\bar{G})$, порожденное характеристическими функциями правых смежных классов. Тогда $L(K, \bar{G})^{*}=\mathbb{C}\left(\chi_{K \sigma} \mid \sigma \in G\right)$. Введем также пространство

$$
\widetilde{L}(K, \bar{G})=L^{\infty}(\bar{G}, \mu) L(K, \bar{G}) \subseteq C^{*}\left(\bar{G} \rtimes L^{\infty}(\bar{G}, \mu)\right) .
$$

Рассмотрим следующие системы операторов [35]:

$$
\begin{aligned}
\mathscr{O}(K, \bar{G}) & =L(K, \bar{G})(L(K, \bar{G}))^{*} \subseteq C^{*}(\bar{G}), \\
\widetilde{\mathcal{O}}(K, \bar{G}) & =\widetilde{L}(K, \bar{G})(\widetilde{L}(K, \bar{G}))^{*} \subseteq C^{*}\left(\bar{G} \rtimes L^{\infty}(\bar{G}, \mu)\right) .
\end{aligned}
$$


В этих формулах произведение вычисляется в $C^{*}$-алгебрах $C^{*}(\bar{G})$ и $C^{*}(\bar{G} \rtimes$ $\left.L^{\infty}(\bar{G}, \mu)\right)$ соответственно.

Тогда ясно, что выполняется следующее:

(i) система $\mathscr{O}(K, \bar{G})$ линейно порождается характеристическими функциями вида $\chi_{\sigma_{1} K \sigma_{2}}, \sigma_{1}, \sigma_{2} \in G$;

(ii) $L(K, \bar{G})$ и $(L(K, \bar{G}))^{*}$ - подпространства в $\mathscr{O}(K, \bar{G})$;

(iii) имеется каноническое спаривание

$$
L(K, \bar{G}) \times L(K, \bar{G}) \rightarrow \mathscr{O}(K, \bar{G}) ;
$$

это спаривание отображает $x, y \in L(K, \bar{G})$ в $x y^{*} \in \mathscr{O}(K, \bar{G})$.

Пусть $\mathscr{E}$ есть $C^{*}$-алгебра. Будем говорить, что линейное отображение $\Phi$ : $\mathscr{O}(K, \bar{G}) \rightarrow \mathscr{E}$ является *-представлением системы операторов $\mathscr{O}(K, \bar{G})$, если

$$
\Phi\left(x y^{*}\right)=\Phi(x)(\Phi(y))^{*}, \quad x, y \in L(K, \bar{G}) .
$$

*-представление для системы операторов $\widetilde{\mathscr{O}}(K, \bar{G})$ является линейным как бимодуль над алгеброй $L^{\infty}(\bar{G}, \mu)$.

Алгебра Гекке $\mathscr{H}_{0}(K, \bar{G})$ есть пересечение $L(K, \bar{G}) \cap(L(K, \bar{G}))^{*}$. Ясно, что она замкнута относительно вышеупомянутой операции спаривания. Очевидно, что *-представление системы операторов $\mathscr{O}(K, \bar{G})$, будучи ограниченным на алгебру Гекке, становится представлением *-алгебры.

Пусть $G, \Gamma, \pi, \pi_{0}, P_{0}, P_{L}$ - такие, как в определении 1. В утверждениях оставшейся части этого раздела для простоты изложения будем считать, что группы $\Gamma, G$ имеют бесконечные нетривиальные классы сопряженности. Из этого предположения следует, что алгебры фон Неймана, ассоциированные ниже с вышеупомянутыми группами, имеют единственные следы.

Мы строим *-представления системы операторов $\mathscr{O}(K, \bar{G})$, принимающие значения в ассоциированных алгебрах фон Неймана, описываемых ниже. Рассмотрим следующие алгебры фон Неймана:

$$
\begin{gathered}
\mathscr{A}=\{\pi(\Gamma)\}^{\prime} \cong \mathscr{R}(\Gamma) \bar{\otimes} B(L) \subseteq \mathscr{B}=\mathscr{R}(G) \bar{\otimes} B(L), \\
\mathscr{A}_{0}=\pi_{0}(\Gamma)^{\prime}=P_{0} \mathscr{A} P_{0} .
\end{gathered}
$$

Заметим, что $\mathscr{A}$ и $\mathscr{B}$ являются алгебрами фон Неймана типа II. Если пространство $L$ бесконечно, то $\mathscr{A}$ и $\mathscr{B}$ - алгебры фон Неймана типа $\mathrm{II}_{\infty} . \mathrm{B}$ представлении для $\{\pi(\Gamma)\}^{\prime}$, введенном в (16), проекция $P_{0}$, которая по условию коммутирует с $\pi(\Gamma)$ и потому принадлежит $\mathscr{A}$, задается формулой

$$
P_{0}=\sum_{\gamma \in \Gamma} \rho(\gamma) \otimes P_{L} \pi_{0}(\gamma) P_{L} \in \mathscr{C}_{1}(\mathscr{A})
$$

Для заданной алгебры фон Неймана $\mathscr{M}$, наделенной точным полуконечным следом $T=T_{\mathscr{M}}$, через $\mathscr{C}_{1}(\mathscr{M})$ обозначаем идеал операторов со следом, ассоциированных с $\mathscr{M}[50]$.

С унитарным представлением $\pi_{0}$ мы связываем *-представление системы операторов, построенное в определении 8. 
Лемма 9. Предположим, что $G, \Gamma, \pi_{0}, P_{0}, P_{L}$ - такие, как в определении 1. Для смежного класса $C=g \bar{\Gamma}_{0}$ в $\bar{G}$ положим по аналогии с формулой (17)

$$
\widetilde{\Phi}_{\pi_{0}, L}(C)=\sum_{\theta \in C} \rho(\theta) \otimes P_{L} \pi_{0}(\theta) P_{L} \in \mathscr{C}_{1}(\mathscr{B}) .
$$

Тогда справедливы следующие утверждения.

(i) Отображение $\widetilde{\Phi}_{\pi_{0}, L}$, ограниченное на $\mathscr{O}(K, G)$, является *-представлением системы операторов $\mathscr{O}(K, G)$. В частности,

$$
\widetilde{\Phi}_{\pi_{0}, L}\left(\chi_{\sigma_{1} K}\right)\left[\widetilde{\Phi}_{\pi_{0}, L}\left(\chi_{\sigma_{2} K}\right)\right]^{*}=\widetilde{\Phi}_{\pi_{0}, L}\left(\chi_{\sigma_{1} K \sigma_{2}^{-1}}\right), \quad \sigma_{1}, \sigma_{2} \in G .
$$

(ii) В силу (17) имеем $\widetilde{\Phi}_{\pi_{0}, L}\left(\chi_{K}\right)=P_{0}$, откуда в силу (i) следует, ито

$$
\widetilde{\Phi}_{\pi_{0}, L}\left(\chi_{\sigma_{1} K}\right)=\widetilde{\Phi}_{\pi_{0}, L}\left(\chi_{\sigma_{1} K}\right) P_{0}, \quad \widetilde{\Phi}_{\pi_{0}, L}\left(\chi_{K \sigma_{1}}\right)=P_{0} \widetilde{\Phi}_{\pi_{0}, L}\left(\chi_{K \sigma_{1}}\right), \quad \sigma_{1} \in G .
$$

(iii) $B$ силу (ii) ограничение $\widetilde{\Phi}_{\pi_{0}, L}$ на алгебру Гекке $\mathscr{H}_{0}(K, \bar{G}) \cong \mathscr{H}_{0}(\Gamma, G)$ принимает значения в алгебре $\mathscr{A}_{0}=P_{0} \mathscr{B} P_{0}$.

(iv) $\left.\widetilde{\Phi}_{\pi_{0}, L}\right|_{\mathscr{H}_{0}(K, \bar{G})}$ сохраняет след $u$, значит, распространяется по непрерывности на $C^{*}$-представление приведенной $C^{*}$-алгебры Гекке $\mathscr{H}_{\mathrm{red}}(\Gamma, G)$ со значениями в $\mathscr{A}_{0}$.

Представление *-алгебр алгебры Гекке из п. (iii) используется для описания операторов Гекке на Г-инвариантных векторах, ассоциированных с диагональным унитарным представлением $\pi_{0} \otimes \pi_{0}^{\text {op }}$ группы $G$. Как было объяснено выше, последнее представление унитарно эквивалентно $\mathrm{Ad} \pi_{0}$, а гильбертово пространство Г-инвариантных векторов канонически отождествляется с $L^{2}$-пространством [50], ассоциированным с коммутантом $\left\{\pi_{n}(\Gamma)\right\}^{\prime}$ алгебры фон Неймана. Формула для операторов Гекке вычисляется в следующей теореме. Случай, когда $\operatorname{dim}_{\mathbb{C}} L=1$, использовался в [37] для получения оценок существенного спектра операторов Гекке, действующих на формах Маасса. Общий случай произвольной размерности Мюррея-фон Неймана был рассмотрен в работе [38].

Операторы Гекке автоматически являются вполне положительными отображениями. Они получаются с помощью математических ожиданий на алгебрах фон Неймана [44], [50]. Пусть

$$
E_{P_{0}(\mathscr{R}(\Gamma) \otimes B(L)) P_{0}}^{P_{0}(\mathscr{R}(G) \otimes B(L)) P_{0}}
$$

- каноническое нормальное условное математическое ожидание, отображающее фактор типа $\mathrm{II}_{1} \quad P_{0}(\mathscr{R}(G) \otimes B(L)) P_{0}$ на подфактор $P_{0}(\mathscr{R}(\Gamma) \otimes B(L)) P_{0}$.

Теорема 10. Оператор Гекке $\Psi([\Gamma \sigma \Gamma])$, ассоииированный с представлением $\mathrm{Ad} \pi_{0}$ и соответствуюший смежному классу $[\Gamma \sigma \Gamma]$ для $\sigma \in G$, является самосопряженным оператором, действующим на пространстве $L^{2}\left(\mathscr{A}_{0}, \tau\right)=$ $L^{2}\left(\left\{\pi_{0}(\Gamma)\right\}^{\prime}, \tau\right)$.

Тогда $\Psi([Г \sigma \Gamma])$ определяется своими значениями на алгебре $\mathscr{A}_{0}$. Для $\sigma \in G$ оператор Гекке $\Psi([\Gamma \sigma \Gamma])$ ставит в соответствие элементу

$$
X \in\left\{\pi_{0}(\Gamma)\right\}^{\prime}=\mathscr{A}_{0}=P_{0} \mathscr{B} P_{0}=P_{0}(\mathscr{R}(\Gamma) \otimes B(L)) P_{0}
$$


onepamop

$$
\Psi([\Gamma \sigma \Gamma])(X)=E_{P_{0}(\mathscr{R}(\Gamma) \otimes B(L)) P_{0}}^{P_{0}(\mathscr{R}(G) \otimes B(L)) P_{0}}\left(\widetilde{\Phi}_{\pi_{0}, L}(\Gamma \sigma \Gamma) X\left(\widetilde{\Phi}_{\pi_{0}, L}(\Gamma \sigma \Gamma)\right)^{*}\right) .
$$

Это теорема 3.2 из [38], обобщающая результаты из [37]. Формулировка адаптирована к рамкам настоящей работы. Данный формализм доказывает, что, как только пространство $L$ выбрано, представление алгебры Гекке является каноническим.

Построим каноническое *-представление $\overline{\mathscr{D} \Phi}$ алгебры Гекке $\mathscr{H}_{0}(K, \bar{G})$ в каноническую "двойную” алгебру (см. ниже) такую, что все представления алгебры Гекке, как в формуле (19), получаются композицией $\overline{\mathscr{D} \Phi}$ с факторотображением.

Мы выполним построение представления $\overline{\mathscr{D} \Phi}$ только в случае, когда размерность фон Неймана $\operatorname{dim}_{\left\{\pi_{0}(\Gamma)\right\}^{\prime \prime}} H_{0}$ равна 1. В этом контексте $\mathscr{A}_{0}$ - это просто алгебра $\mathscr{R}(\Gamma)$. Рассуждение легко распространить и на случай размерности фон Неймана, большей 1 , но формулы будут более сложными.

Тот факт, что размерность фон Неймана алгебры фон Неймана типа II, порожденной $\pi_{0}(\Gamma)$, равна 1 , означает, что представление $\left.\pi\right|_{\Gamma}$ допускает циклический следовой вектор $\zeta$. В этом случае $*$-представление $\widetilde{\Phi}_{\pi_{0}, L}$, введенное в лемме 9, заменяется на построенное ниже представление $t$ канонической системы, введенной в определении 8 , принимающее значения в $\mathscr{L}(G)$. Напомним, что алгебра $\mathscr{L}(G)$ антиизоморфна $\mathscr{R}(G)$.

Для $\sigma_{1}, \sigma_{2} \in G$ и для подмножества группы $G$ вида $A=\sigma_{1} \Gamma \sigma_{2}$ положим

$$
t\left(\sigma_{1} K \sigma_{2}\right)=\sum_{\theta \in A} \overline{\left\langle\pi_{0}(\theta) \zeta, \zeta\right\rangle} \lambda_{\theta} \in \mathscr{L}(G) .
$$

Тогда по лемме 9, полагая пространство $L$ равным $\mathbb{C} \zeta$, мы выводим (см. замечание 42$)$, что $t$ определяет $*$-представление системы операторов $\mathscr{O}(K, G)$ со значениями в алгебре фон Неймана $\mathscr{L}(G)$ (комплексное сопряжение в формуле $(20)$ связано с тем, что через канонический антиизоморфизм мы переключаемся с алгебры $\mathscr{R}(G)$ на алгебру $\mathscr{L}(G))$.

Алгебра Гекке *-представления $\Psi$, введенная в (19), теперь принимает значения в $\mathscr{L}(\Gamma)$ и задается формулой

$$
\Psi([\Gamma \sigma \Gamma])(X)=E_{\mathscr{L}(\Gamma)}^{\mathscr{L}(G)}\left(t(\Gamma \sigma \Gamma) X(t(\Gamma \sigma \Gamma))^{*}\right), \quad \sigma \in G .
$$

Линейный оператор $\Psi([\Gamma \sigma \Gamma]), \sigma \in G$, очевидным образом продолжается на пространство Гельфанда-Неймарка-Сигала $\ell^{2}(\Gamma)$ (см., например, [50]), рассматриваемое относительно канонического следа.

Все операторы Гекке $\Psi([\Gamma \sigma \Gamma]), \sigma \in G$, являются вполне положительными и унитальными. Отсюда следует, что представление в (21)) продолжается, забывая структуру алгебры на пространстве образа, до $*$-представления $\Psi_{0}$ алгебры $\mathscr{H}_{0}(G, \Gamma)$ со значениями в $B\left(\ell^{2}(\Gamma) \ominus \mathbb{C} 1\right)$. Как объясняется в примеpe 29 (см. также [37], [39]), справедливость гипотезы Рамануджана-Петерссона на формах Маасса в частном случае $G=\operatorname{PGL}(2, \mathbb{Z}[1 / p])$ эквивалентна тому, что представление $\Psi_{0}$ продолжается до представления приведенной $C^{*}$-алгебры Гекке $\mathscr{H}_{\text {red }}(G, \Gamma)$. 
Мы докажем, что представление $\Psi$ алгебры Гекке, введенное в (21), получается из канонического представления $\overline{\mathscr{D} \Phi}$ алгебры Гекке.

Введем следующие два скрещенных произведения $C^{*}$-алгебр. Они являются расширением с двумя переменными алгебр Роэ [43], [6].

ОПРЕДЕЛЕНиЕ 11. Рассмотрим скрещенные произведения $C^{*}$-алгебр:

$$
\begin{aligned}
& \mathscr{D} \mathscr{R}(G)=\chi_{K}\left(C^{*}\left(\left(G \times G^{\mathrm{op}}\right) \rtimes L^{\infty}(\bar{G}, \mu)\right)\right) \chi_{K}, \\
& \mathscr{D} \mathscr{R}(\bar{G})=\chi_{K}\left(C^{*}\left(\left(\bar{G} \times \bar{G}^{\mathrm{op}}\right) \rtimes L^{\infty}(\bar{G}, \mu)\right)\right) \chi_{K} .
\end{aligned}
$$

$C^{*}$-алгебра $\mathscr{D} \mathscr{R}(G)$ обладает каноническим представлением типа Купмана $\alpha$ в $B\left(\ell^{2}(\Gamma)\right)$, получающимся следующим образом: пусть $G \times G^{\text {op }}$ действует как группоид на $Г$ левым и правым умножением, причем операция определена, как только результат умножения принадлежит $Г$. С другой стороны, $C(K)$ действует умножением на $l^{\infty}(\Gamma)$ и потому на $\ell^{2}(\Gamma)$.

Заметим, что алгебры, введенные в определении 11, являются углами (приведенными проекцией $\left.\chi_{K} \in L^{\infty}(\bar{G}, \mu)\right)$ в большем скрещенном произведении $C^{*}$-алгебр, соответствующими сохраняющим меру действиям $G \times G^{\text {op }}$ и $\bar{G} \times \bar{G}^{\text {op }}$ на $\bar{G}$. Поскольку действие сохраняет меру, эти две алгебры обладают очевидными соответствующими приведенными скрещенными произведениями $C^{*}$-алгебр.

Приводимая ниже теорема 12 утверждает, что представление алгебр Гекке $\Psi$, введенное в $(21)$, которое на самом деле является представлением алгебр Гекке, ассоциированным с представлением $\pi_{0} \otimes \pi_{0}^{\text {op }}$, получается из внутреннего представления алгебры Гекке (обозначаемого через $\mathscr{D} \Phi$ ) со значениями в "двойной" алгебре $\mathscr{D} \mathscr{R}(G)$. Чтобы получить представление алгебр Гекке $\Psi$, берут композицию представления $*$-алгебр $\mathscr{D} \Phi$ с представлением типа Купмана $\alpha$ алгебры $\mathscr{D} \mathscr{R}(G)$, введенным в определении 11.

Так как представление *-алгебр $\mathscr{D} \Phi$ продолжается (ввиду того, что оно сохраняет след) на приведенную $C^{*}$-алгебру Гекке, препятствие (если оно есть) к продолжению представления $\Psi_{0}$ на приведенную $C^{*}$-алгебру Гекке $\mathscr{H}_{\text {red }}(G, \Gamma)$ (которое, как пояснено выше, в случае представления $\pi_{n}$ является препятствием к выполнению гипотезы Рамануджана-Петерссона на формах Маaсcа [37]) лежит в ядре вышеупомянутого канонического представления $\alpha$, пересеченном с образом представления $\mathscr{D} \Phi$.

Если $f$ - функция на $\bar{G}$, обозначим соответствующий сверточный элемент в $C^{*}(\bar{G})$ через $L(f)$. Имеет место следующая теорема.

Teopema 12. (i) Cоответствие

$$
[K \sigma K] \rightarrow \chi_{K}\left(L\left(\chi_{K \sigma K}\right) \otimes L\left(\chi_{K \sigma K}\right)^{\mathrm{op}}\right) \chi_{K}, \quad \sigma \in G,
$$

продолжается по линейности на *-алгебру представления $\overline{\mathscr{D} \Phi}$ алгебры Гекке $\mathscr{H}_{0}(K, G)$ в $\mathscr{D} \mathscr{R}(\bar{G})$. Это представление сохраняет след, и, следовательно, оно очевидным образом продолжается на $\mathscr{H}_{\mathrm{red}}(\Gamma, G)$, когда значения рассматриваются в приведенной $C^{*}$-алгебре со скрещенным произведением, ассочиированной с $\mathscr{D} \mathscr{R}(\bar{G})$. 
(ii) *-представление $t$ системы операторов $\mathscr{O}(K, \bar{G})$, введенное в $(20)$, продолжается до $\mathscr{D} \mathscr{R}(G)$-значного *-представления $t_{2}$ системы операторов $\mathscr{O}$, содержащейся в $\mathscr{D} \mathscr{R}(\bar{G})$. Система операторов $\mathscr{O}$ содержит образ $\overline{\mathscr{D} \Phi}$.

(iii) Композиция $\mathscr{D} \Phi=t_{2} \circ \overline{\mathscr{D}} \Phi$, действующая по формуле

$$
[\Gamma \sigma \Gamma] \rightarrow \chi_{K}\left(t(\Gamma \sigma \Gamma) \otimes(t(\Gamma \sigma \Gamma))^{\mathrm{op}}\right) \chi_{K},
$$

продолжается до представления *-алгебр алгебры Гекке $\mathscr{H}_{\mathrm{red}}(\Gamma, G)$ в приведенную $C^{*}$-алгебру скрещенного произведения, ассоциированную с $\mathscr{D} \mathscr{R}(G)$.

(iv) Пусть $\alpha$ - представление из определения 11, и пусть $\Psi$ - алгебра Гекке, введенная формулой (21). Тогда

$$
\Psi=\alpha \circ \mathscr{D} \Phi=\alpha \circ t_{2} \circ \overline{\mathscr{D} \Phi} .
$$

Автор глубоко признателен Р.И.Григорчуку, А. Любоцки и Л. Паунеску, воодушевившим его на написание этой работы. Автор выражает искреннюю благодарность У. Хаагерупу, П. де ля Арпу, П. Куцко, Х. Московичи, Р. Несту, Т. Стигеру, Полу Гаретту, Виченциу Пашолу, Александру Попа и пользователю plusepsilon.de на mathoverflow за ряд комментариев по вопросам, связанным с этой работой, Р. И. Григорчуку и Татьяне Смирновой-Нагнибеда за обсуждения по теме этой работы и, в частности, по центральным характерам группы, Дж. Петерссону и А. Тому за объяснение значения результатов работы [33], Н. Озаве за предоставление автору для опубликования его отредактированных заметок [40] по работе автора [37], а также Ф. Бока за комментарии по этой работе и за помощь в улучшении стиля изложения. Автор благодарен факультету математических наук Копенгагенского университета за теплый прием на последних этапах этой работы.

\section{2. Схема работы}

Мы описываем схему построения гильбертовых пространств "виртуальных" $\Gamma$-инвариантных векторов и унитарного действия группы $G$ на индуктивном пределе этих пространств. Напомним (см. введение), что $\Gamma-$ почти нормальная подгруппа группы $G$, т. е. для всех $\sigma \in G$ группа $\Gamma_{\sigma}=\sigma \Gamma \sigma^{-1} \cap \Gamma$ имеет конечный индекс в Г. В этой работе мы предполагаем, что для всех $\sigma \in G$ значения групповых индексов $\left[\Gamma: \Gamma_{\sigma}\right]$ и $\left[\Gamma: \Gamma_{\sigma^{-1}}\right]$ равны. Это предположение выполняется автоматически, если $\Gamma$ - группа с бесконечными нетривиальными классами сопряженности при наличии представления $\pi_{0}$ как в определении 1 (например, в силу теории индексов Джоунса [25]). Мы также предполагаем, что семейство $\mathscr{S}$, порожденное через операцию пересечения подгруппами $\Gamma_{\sigma}$, $\sigma \in G$, разделяет точки из $Г$. Пусть $K$ - проконечное пополнение группы $Г$ относительно семейства $\mathscr{S}$.

Сначала рассмотрим унитарное представление $\pi$ группы $G$, обладающее свойствами (i), (ii) из определения 1 . В этом случае $\left.\pi\right|_{\Gamma}$ является целым кратным регулярного представления. Чтобы построить Г-инвариантные векторы, предположим, что $H$ содержится в большем векторном пространстве $\mathscr{V}$ и что представление $\pi$ продолжается до представления $\pi_{\mathscr{V}}$ группы $G$ в группу 
линейных изоморфизмов пространства $\mathscr{V}$. Мы предполагаем, что пространство $\mathscr{V} \Gamma$, состоящее из векторов пространства $\mathscr{V}$, неподвижных относительно $\pi_{\mathscr{V}}(\Gamma)$, нетривиально. Чтобы построить представление $\bar{\pi}^{\mathrm{p}}$, одновременно рассматриваются пространства $\mathscr{V}^{\Gamma_{0}}$ векторов из $\mathscr{V}$, неподвижных относительно действия $\pi_{\mathscr{V}}\left(\Gamma_{0}\right)$, где $\Gamma_{0}-$ любая группа, сопряженная в $G$ с подгруппой из $\mathscr{S}$. Заметим, что $\pi_{\mathscr{V}}(\sigma)$ для $\sigma \in G$ переводит векторное пространство $\mathscr{V} \Gamma_{0}$ B $\mathscr{V} \sigma \Gamma_{0} \sigma^{-1}$.

Мы строим структуру гильбертова пространства на пространствах $\mathscr{V} \Gamma_{0}$, которая определяет гильбертовы пространства $H^{\Gamma_{0}}$ векторов, неподвижных относительно $\Gamma_{0}, \Gamma_{0} \in \mathscr{S}$. На скалярное произведение в пространствах инвариантных векторов мы налагаем следующее условие: включения

$$
H^{\Gamma_{0}} \subseteq H^{\Gamma_{1}}, \quad \Gamma_{0}, \Gamma_{1} \in \mathscr{S}, \quad \Gamma_{1} \subseteq \Gamma_{0},
$$

являются изометрическими и $\pi_{\mathscr{V}}(\sigma)$ для $\sigma \in G$ изометрически отображает $H^{\Gamma_{0} \cap \Gamma_{\sigma}-1}$ на $H^{\sigma \Gamma_{0} \sigma^{-1} \cap \Gamma_{\sigma}}$.

Используя эту процедуру, получаем (см. формулу (35)) структуру предгильбертова пространства на объединении $\bigvee H^{\Gamma_{0}}$. Пусть $\bar{H}^{\mathrm{p}}-$ гильбертово пространство пополнения этого объединения. Ясно, что $\pi_{\mathscr{V}}$ индуцирует представление группы $G$ в изоморфизмы пространства $\bigvee_{\Gamma_{0} \in \mathscr{S}} \mathscr{V}^{\Gamma_{0}}$.

Из условий, налагаемых нами на скалярные произведения в гильбертовых пространствах $H^{\Gamma_{0}}, \Gamma_{0} \in \mathscr{S}$, следует, что $\pi_{\mathscr{V}}$ индуцирует унитарное представление $\bar{\pi}^{\mathrm{p}}$ группы $G$ в унитарную группу $\mathscr{U}\left(\bar{H}^{\mathrm{p}}\right)$ гильбертова пространства $\bar{H}^{\mathrm{p}}$. Обозначение - р над $\pi$ означает вышеупомянутую операцию пополнения, состоящую в переходе от $\pi$ к унитарному представлению $\bar{\pi}^{\mathrm{p}}$ на пополненном гильбертовом пространстве объединения пространств $\Gamma_{0}$-инвариантных векторов, $\Gamma_{0} \in \mathscr{S}$.

Напомним, что пополнение Шлихтинга $\bar{G}$ (см., например, [48], [51], [26], [29]) дискретной группы $G$ относительно подгрупп из $\mathscr{S}$ является локально компактной вполне несвязной группой, полученной как объединение непересекающихся двойных смежных классов $K \sigma K$, с очевидным отношением умножения, где Г $\sigma \Gamma$ пробегает множество представителей двойных смежных классов для Г в $G$ (см. также [4], [7], [21]).

Пусть $d_{\pi}=\operatorname{dim}_{\{\pi(\Gamma)\}^{\prime \prime}} H_{0} \in \mathbb{N}$ - размерность фон Неймана пространства $H_{0}$ как модуля над фактором типа II $\{\pi(\Gamma)\}^{\prime \prime}$ (определения и обозначения см. в [19; $\S 3.3])$. Мы докажем (лемма 26), что левое регулярное представление $\lambda_{K}$ группы $K$ в унитарную группу $\mathscr{U}\left(L^{2}(K, \mu)\right)$ имеет кратность $d_{\pi}$ в $\left.\bar{\pi}^{\mathrm{P}}\right|_{K}$.

Пусть $\mu$ - нормированная мера Хаара на $K$; продолжим ее на локально компактную группу $\bar{G}$ - до меры Хаара $\mu$, нормированной условием $\mu(K)=1$. Из предположения, что $\left[\Gamma: \Gamma_{\sigma}\right]=\left[\Gamma: \Gamma_{\sigma^{-1}}\right]$ для всех $\sigma$ в $G$, следует, что мера $\mu$ бивариантна относительно левых и правых сдвигов элементами группы $\bar{G}$.

Распространим построение вышеупомянутого унитарного представления на случай, когда $\pi_{0}$ - унитарное представление из определения 1 . В этом случае унитарное представление $\left.\pi\right|_{\Gamma}$ уже не является кратным левого регулярного представления группы Г, но оно является подпредставлением большего представления $\pi$ с упомянутыми выше свойствами. 
Мы доказываем, что описанное выше построение, которое мы провели для того, чтобы получить унитарное представление $\bar{\pi}^{\mathrm{p}}$, можно повторить для $\pi_{0}$. Мы получаем унитарное представление $\bar{\pi}_{0}^{\mathrm{p}}$ группы $\bar{G}$, ассоциированное с действием исходной группы $G$ на пространствах $\Gamma_{0}$-инвариантных векторов, $\Gamma_{0} \in \mathscr{S}$. Представление $\bar{\pi}_{0}^{\mathrm{p}}$ естественным образом продолжается на $C^{*}$-алгебру локально компактной группы $\bar{G}$. Оно также продолжается до представления $C^{*}$-алгебр полной $C^{*}$-алгебры, ассоциированной одновременно с группами $G$ и $\bar{G}$ (см. определение 16).

В теореме 34 отмечается, что “клеточно-матричные коэффициенты” представления $\bar{\pi}_{0}^{\mathrm{p}}$ являются операторами Гекке, ассоциированными с представлением $\bar{\pi}_{0}^{\mathrm{p}}$ и соответствующими уровню $\Gamma_{0}$, где группа $\Gamma_{0} \in \mathscr{S}$ определяется размером "клетки". Мы используем это в теореме 2, чтобы найти явную формулу (см. формулу (4)) для операторов Гекке в терминах параметров из представления $\pi_{0}$.

Один из результатов этой работы - связь между представлениями $\pi_{0}$ и $\bar{\pi}_{0}^{\mathrm{p}}$. Представление $\bar{\pi}_{0}^{\mathrm{p}}$ является представлением типа I группы $\bar{G}$. Поэтому оно имеет ассоциированный характер [22], [45], [17], который мы обозначаем через $\theta_{\bar{\pi}_{0}^{\mathrm{p}}}=$ "Tr" $\bar{\pi}_{0}^{\mathrm{p}}$. Мы доказываем (см. следствие 4 ), что значения характеpa $\theta_{\bar{\pi}_{0}^{\mathrm{p}}}(g)$, вычисленные на $g \in G$, определяются суммированием по смежным классам значений положительно определенной функции $\phi_{0}$ на $G$, введенной в (6). Та же положительно определенная функция определяет формулу для “Tr $\pi_{0}(g)$ " исходного представления $\pi_{0}$.

Основная модель унитарных представлений, рассмотренных выше, - представление пространств $\Gamma_{0}$-инвариантных векторов в случае левого регулярного представления $\lambda_{G}$ группы $G$ в $\mathscr{U}\left(l^{2}(G)\right)$ (более подробное изложение см. в примере 27 раздела 4). В этом случае гильбертовы пространства $H^{\Gamma_{0}}, \Gamma_{0} \in \mathscr{S}$, являются гильбертовыми пространствами $l^{2}\left(\Gamma_{0} \backslash G\right)$ со скалярным произведением, нормированным так, что включения $l^{2}\left(\Gamma_{0} \backslash G\right) \subseteq l^{2}\left(\Gamma_{1} \backslash G\right)$ изометричны для $\Gamma_{1} \subseteq \Gamma_{0}$. Гильбертово пространство $\bar{H}^{\mathrm{p}}$ есть просто $L^{2}(\bar{G}, \mu)$, и в этом частном случае представление $\bar{\pi}$ р есть просто левое регулярное представление группы $\bar{G}$ в унитарную группу $\mathscr{U}\left(L^{2}(\bar{G}, \mu)\right)$.

Напомним (см. раздел 1$)$, что алгебра Гекке $\mathscr{H}_{0}(\Gamma, G)$ двойных смежных классов подгруппы $\Gamma$ в $G$ имеет каноническое вложение $*$-алгебр в $B\left(\ell^{2}(\Gamma \backslash G)\right)$. Ее замыкание в равномерной норме является $C^{*}$-алгеброй $\mathscr{H}_{\text {red }}(\Gamma, G)$, называемой приведенной $C^{*}$-алгеброй Гекке - по аналогии с приведенной $C^{*}$-алгеброй дискретной группы [7], [20], [51], [2], [14]). Представление

$$
\mathscr{H}_{\text {red }}(\Gamma, G) \subseteq B\left(\ell^{2}(\Gamma \backslash G)\right)
$$

называется левым регулярным представлением алгебры Гекке. Коммутант порождается правым квазирегулярным представлением $\rho_{\Gamma \backslash G}$ группы $G$ в унитарную группу пространства $\ell^{2}(\Gamma \backslash G)$ (см., например, [7]).

Содержание проблемы Рамануджана-Петерссона состоит в нахождении ограничений на рост матричных коэффициентов и собственных значений для представлений алгебры Гекке на гильбертовых пространствах Г-инвариантных 
векторов, ассоциированных с унитарным представлением $\pi$ группы $G$, рассмотренным выше. Гипотеза Рамануджана-Петерссона фактически предлагает ответ на вопрос [37], когда матричные коэффициенты представления алгебры $\mathscr{H}_{0}(\Gamma, G)$, ассоциированного с унитарным представлением $\pi$, являются слабыми пределами выпуклых комбинаций матричных коэффициентов алгебры Гекке для левого регулярного представления алгебры Гекке.

В терминологии данной работы это эквивалентно выяснению, когда сферические функции (матричные коэффициенты, соответствующие векторам, неподвижным относительно $K$ ), ассоциированные с представлением $\bar{\pi}_{0}^{\mathrm{p}}$ группы $\bar{G}$, слабо содержатся в левом регулярном представлении алгебры Гекке. Ясно, что это эквивалентно тому, что представление $\bar{\pi}_{0}^{\mathrm{p}}$ слабо содержится в левом регулярном представлении группы $\bar{G}$, действующей левыми сдвигами на $L^{2}(\bar{G}, \mu)$. Гипотеза Рамануджана-Петерссона доказана для автоморфных форм П. Делинем [15]. Для форм Маасса [31] в общей постановке проблема открыта (см. [49], [47], [24], [11], [37]). Мы формулируем следующую проблему.

ПроблемА (обобщенная проблема Рамануджана-Петерссона). Найти условия на представление $\pi_{0}$, при которых $\bar{\pi}_{0}^{\mathrm{p}}$ слабо содержится в левом регулярном представлении $\lambda_{\bar{G}}$ группь $\bar{G}$ на $L^{2}(\bar{G}, \mu)$. (Достаточно найти условия на унитарное представление $\pi$ группы $G$, при которых унитарное представление $\left.\bar{\pi}_{0}^{\mathrm{P}}\right|_{G}$ группы $G$ слабо содержстся в унитарном представлении $\left.\lambda_{\bar{G}}\right|_{G}$.)

В случае унитарного представления $\pi_{n}$ группы $G=\operatorname{PGL}(2, \mathbb{Z}[1 / p])(p-$ простое число, $n \in \mathbb{N}$ ), полученного ограничением из дискретной серии представлений группы $\mathrm{PSL}(2, \mathbb{R})$, представления $\bar{\pi}_{n}^{\mathrm{p}}$ содержат всю информацию группового гармонического анализа о пространствах автоморфных форм (здесь группа $\Gamma$ - это $\operatorname{PSL}(2, \mathbb{Z}))$. Сферические матричные коэффициенты группы $\bar{\pi}_{n}^{\mathrm{p}}$ кодируют информацию о собственных значениях операторов Гекке.

Ниже мы приводим примеры представлений $\pi$ и ассоциированного представления $\bar{\pi}$. Более детальное изложение см. в разделе 4 . Самый простой случай когда указанное выше унитарное представление $\pi$ группы $G$ имеет также то свойство, что $\left.\pi\right|_{\Gamma}$ есть целое кратное левого регулярного представления группы Г. В терминологии размерности Мюррея-фон Неймана (см. [19; § 3.3]) это случай, когда $\operatorname{dim}_{\{\pi(\Gamma)\}^{\prime \prime}} H$ - целое число. В этом случае, как объяснено выше, существует гильбертово подпространство $L$ пространства $H$ со свойством, вводимым следующим определением.

ОПРЕдЕлЕниЕ 13. Пусть $\pi$ - указанное выше унитарное представление группы $G$. Рассмотрим подпространство $L$ пространства $H$ такое, что $\pi(\gamma) L$ ортогонально $L$ для $\gamma \neq e$. Назовем такое пространство Г-блуждающим подпространством для $\pi$. Если вдобавок $H=\bigvee_{\gamma \in \Gamma} \pi(\gamma) L$, то такое подпространство будем называть Г-блуждающим порождающим подпространством.

В описанном выше контексте гильбертовы пространства $\Gamma_{0}$-инвариантных векторов канонически отождествляются с гильбертовыми пространствами $L \otimes$ $l^{2}\left(\Gamma_{0} \backslash G\right), \Gamma_{0} \in \mathscr{S}$. Напомним, что $K-$ проконечное пополнение группы $\Gamma$ относительно подгрупп из $\mathscr{S}$. Пространство $\bar{H}^{\mathrm{p}}$ отождествляется с $L \otimes L^{2}(K, \mu)$. Проблема состоит в отождествлении представления групп $G$ и $\bar{G}$ на $\bar{H}^{\mathrm{p}}$. 
Описанная выше ситуация имеет место, например, в случае (см. пример 28), когда $(\mathscr{X}, \nu)$ - бесконечномерное пространство, на котором $G$ действует сохраняющими меру преобразованиями. В этом случае $H=L^{2}(\mathscr{X}, \nu)$ и $\pi-$ представление Купмана $\pi_{\text {Коор }}$ (см., например, [27]):

$$
\left(\pi_{\text {Koop }}(g) f\right)(x)=f\left(g^{-1} x\right), \quad x \in \mathscr{X}, \quad g \in G, \quad f \in L^{2}(\mathscr{X}, \nu) .
$$

Предположим, что ограничение действия группы $G$ на $\Gamma$ допускает фундаментальную область $F$. Тогда можно положить $L=L^{2}\left(F,\left.\nu\right|_{F}\right)$. Это Г-блуждающее порождающее подпространство, ассоциированное с представлением $\pi_{\text {Коор }}$. В этом случае $\operatorname{dim}_{\left\{\pi_{\text {Коор }}(\Gamma)\right\}^{\prime \prime}} H=\infty$. Тогда $\bar{H}^{\mathrm{p}}=L^{2}\left(F,\left.\nu\right|_{F}\right) \otimes L^{2}(K, \mu)$. Представление $\bar{\pi}_{\text {Коор }}^{\mathrm{p}}$ определяется Г-значным коциклом на $G \times F$, определяемым действием группы $G$ на $\mathscr{X}$ при отождествлении $\mathscr{X} \cong F \times \Gamma$. Чтобы получить представление $\bar{\pi}_{\text {Коop }}^{\mathrm{p}}$, этот коцикл рассматривают как имеющий значения в $K$.

Пусть $\pi_{0}$ - представление группы $G$, обладающее свойствами, перечисленными в определении 1. Они обычно выполняются, когда $\operatorname{dim}_{\left\{\pi_{0}(\Gamma)\right\}^{\prime \prime}} H$ не целое. А последнее имеет место, когда Г-инвариантные векторы являются автоморфными формами.

В этом случае $G=\operatorname{PGL}(2, \mathbb{Z}[1 / p]), p$ - простое, $Г$ - модулярная группа и $H_{n}=H^{2}\left(\mathbb{H}, \nu_{n}\right)$. Здесь $\nu_{n}-$ это мера $(\operatorname{Im} z)^{n-2} d \bar{z} d z$ на верхней полуплоскости $\mathbb{H}$. Представления $\pi_{0}=\pi_{n}$ получаются ограничением на $G$ представлений из дискретной серии $\left(\pi_{n}\right)_{n \in \mathbb{N}, n \geqslant 2}$ унитарных представлений группы $\operatorname{PSL}_{2}(\mathbb{R})$ (см., например, [30]). В этом случае нет канонического блуждающего подпространства $L$, поскольку, как доказано в [19; §3.3.d],

$$
\operatorname{dim}_{\left\{\pi_{n}(\Gamma)\right\}^{\prime \prime}} H_{n}=\operatorname{dim}_{\Gamma} H_{n}=\frac{n-1}{12} .
$$

Основанием для предыдущего утверждения о несуществовании служит то, что если такое пространство $L$ существует, то

$$
\operatorname{dim}_{\mathbb{C}} L=\operatorname{dim}_{\Gamma} H_{n} .
$$

Это невозможно, если $(n-1) / 12$ не целое.

В теории автоморфных форм проблема построения гильбертовых пространств Г-инвариантных векторов решается с помощью фундаментальной области и скалярного произведения Петерссона [34], которое состоит в интегрировании по фундаментальной области. В рамках этой работы мы заменяем интегрирование по фундаментальной области действием проекции $P_{L}$ на пространство $L$.

Предполагается, что существует унитарное представление $\widehat{\pi}_{n}$ на большем гильбертовом пространстве, содержащее $\pi_{n}$ в качестве подпредставления из определения 1. В этом случае (см. пример 29) большее гильбертово пространство - это $H=L^{2}\left(\mathbb{H}, \nu_{n}\right)$. Унитарное представление $\widehat{\pi}_{n}$ действует на функциях на $\mathbb{H}$ по той же формуле, что и $\pi_{n}$. Из свойства инвариантности меры $\nu_{n}$ следует, как и в случае $\pi_{n}$, что $\widehat{\pi}_{n}$ является унитарным представлением группы $G$.

Мы пользуемся обозначениями из определения 1 . Пусть $P_{0}$ - ортогональная проекция из $L^{2}\left(\mathbb{H}, \nu_{n}\right)$ на пространство $H_{n}$ суммируемых с квадратом аналитических функций. Имеем $\left[P_{0}, \widehat{\pi}_{n}(g)\right]=0$ для всех $g \in G$. Отсюда следует, 
что $\pi_{n}(g)=P_{0} \widehat{\pi}_{n}(g) P_{0}, g \in G$. Возьмем пространство $L=L^{2}\left(F, \nu_{n}\right)$ в качестве канонического выбора Г-блуждающего порождающего подпространства $L$ для $\widehat{\pi}_{n}$. Напомним, что $P_{L}$ - ортогональная проекция на $L$. Очевидно, что в этом случае $P_{L}$ - это в точности $M_{\chi_{F}}$, оператор умножения на характеристическую функцию фундаментальной области $F$, действующий на $L^{2}\left(\mathbb{H}, \nu_{n}\right)$.

Из вычислений в $[19 ; \S 3.3]$ следует, что произведение $P_{0} M_{\chi_{F}}$ является оператором со следом, причем его след равен размерности Мюррея-фон Неймана $\operatorname{dim}_{\left\{\pi_{n}(\Gamma)\right\}^{\prime \prime}} H_{n}$.

Чтобы абстрактно определить пространство Г-инвариантных векторов, мы используем относительное положение (операторный угол) проекций $P_{0}$ и $M_{\chi_{F}}$. В этой ситуации техническое условие (определение 1) состоит в сходимости в пространстве операторов Гильберта-Шмидта рядов

$$
\sum_{\theta \in \Gamma \sigma \Gamma} P_{L} \pi_{n}(\theta) P_{L}, \quad \sigma \in G .
$$

В рассматриваемом примере это условие выполняется, так как воспроизводящее ядро для проекции на пространство автоморфных форм и воспроизводящие ядра ассоциированных операторов Гекке являются суммой операторных ядер (воспроизводящих ядер Березина [3], [36]) операторов

$$
\sum_{\theta \in \Gamma \sigma \Gamma} M_{\chi_{F}} \pi_{n}(\theta) M_{\chi_{F}}, \quad \sigma \in G .
$$

Более того, сумма следов соответствующих операторов также абсолютно сходится. Это следует из вычислений в [53] и [19; §3.3].

Мы доказываем (см. теорему 34), что сумма (26) является проекцией, когда сумма берется по Г (например, $\sigma$ - единичный элемент). Мы показываем также, что образ этой проекции (который является подпространством из $L$ ) унитарно эквивалентен гильбертову пространству Г-инвариантных векторов. Более того, та же унитарная эквивалентность преобразует оператор Гекке, соответствующий двойному смежному классу $[\Gamma \sigma \Gamma]$, в сумму $(26)$.

Преимущество этой точки зрения на пространства автоморфных форм состоит в том, что формула (26) делает возможным прямое вычисление следов операторов Гекке на любом уровне $\Gamma_{0} \in \mathscr{S}$. Это используется для того, чтобы вычислить (следствие 7 ) значения характера $\theta_{\bar{\pi}_{n}^{\mathrm{p}}}(g), g \in G$. Эти значения являются частичными суммами следов операторов, имеющими вид (26) (см. теорему 34 и замечание 35$)$.

Пространства Г-инвариантных векторов для унитарного представления $\overline{\pi_{\text {Kоop }}} \mathrm{p}$ можно получить другим способом, если вышеупомянутое представление допускает “квадратный корень", как описано ниже (см. пример 30 в разделе 4). Пусть пространством бесконечной меры будет $\mathbb{H}$, наделенное $\operatorname{PSL}(2, \mathbb{R})$-инвариантной мерой $d \nu_{0}(z)=(\operatorname{Im} z)^{-2} d \bar{z} d z$. Пусть $\pi=\pi_{\text {Коор }}-$ соответствующее унитарное представление Купмана группы $\operatorname{PSL}(2, \mathbb{R})$ в унитарную группу пространства $L^{2}\left(\mathbb{H}, \nu_{0}\right)$. Обозначим через $\pi_{n}^{\text {op }}$ сопряженное представление представления $\pi_{n}$. В силу метода квантизации Березина (см. [42], [3]) представление $\pi_{\text {Коор }}$ факторизуется следующим образом:

$$
\pi_{\text {Koop }} \cong \pi_{n} \otimes \pi_{n}^{\text {op }} .
$$


Как и в предыдущем примере, пусть $G=\operatorname{PGL}(2, \mathbb{Z}[1 / p]), p$ - простое, а $\Gamma$ -

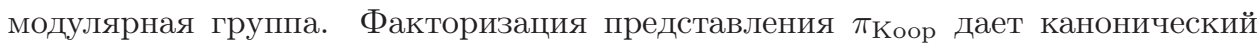
выбор для гильбертовых пространств $\Gamma_{0}$-инвариантных векторов, $\Gamma_{0} \in \mathscr{S}$. Действительно, представление $\pi_{n} \otimes \pi_{n}^{\text {op }}$ унитарно эквивалентно присоединенному представлению $\mathrm{Ad} \pi_{n}(g)$ в унитарную группу операторов Гильберта-Шмидта $\mathscr{C}_{2}\left(H_{n}\right) \cong H_{n} \otimes \bar{H}_{n}^{\mathrm{p}}$.

Большее векторное пространство, содержащее $\mathscr{C}_{2}\left(H_{n}\right)$, - это $\mathscr{V}=B\left(H_{n}\right)$, пространство ограниченных линейных операторов на $H_{n}$. Тогда присоединенное представление $\mathrm{Ad} \pi_{n}(g)$ продолжается до представления в группу внутренних автоморфизмов алгебры $B\left(H_{n}\right)$. Пространство $\Gamma_{0}$-инвариантных векторов $\mathscr{V}^{\Gamma_{0}}$ в этой ситуации является фактором типа $\mathrm{II}_{1}$ :

$$
\mathscr{A}_{n}\left(\Gamma_{0}\right)=\left\{\pi_{n}\left(\Gamma_{0}\right)\right\}^{\prime}=\left\{X \in B\left(H_{n}\right) \mid\left[X, \pi_{n}(\gamma)\right]=0, \gamma \in \Gamma_{0}\right\} .
$$

Тот факт, что коммутант $\mathscr{A}_{n}\left(\Gamma_{0}\right)$ является фактором типа $\mathrm{II}_{1},-$ следствие того, что $\operatorname{dim}_{\Gamma} H_{n}$ конечна (см. [19; § 3.3.d]).

Тогда гильбертово пространство $H^{\Gamma_{0}}$ - это просто $L^{2}\left(\mathscr{A}_{n}\left(\Gamma_{0}\right), \tau\right)$, гильбертово пространство Гельфанда-Наймарка-Сигала, ассоциированное с единственным следом $\tau$ на $\mathscr{A}_{n}\left(\Gamma_{0}\right)$. Семейство $\left\{\mathscr{A}_{n}\left(\Gamma_{0}\right)\right\}_{\Gamma_{0} \in \mathscr{S}}$ является направленным семейством факторов типа $\mathrm{II}_{1}$. Пусть $\mathscr{A}_{n}^{\infty}$ - фактор типа $\mathrm{II}_{1}$, полученный как индуктивный предел этого направленного семейства факторов типа $\mathrm{II}_{1}$. Обозначим через $\tau$ единственный след на $\mathscr{A}_{n}^{\infty}$.

Тогда пространство $\bar{H}^{\mathrm{p}}$ есть $L^{2}\left(\mathscr{A}_{n}^{\infty}, \tau\right)$, а $\overline{\mathrm{Ad} \pi_{n}} \mathrm{p}$ - продолжение представления $\operatorname{Ad} \pi_{n}(g)$. В теореме 10 [38; теорема 3.2] мы доказали, что $K$-сферические матричные коэффициенты для $\overline{\mathrm{Ad} \pi_{n}} \mathrm{p}$ явно вычисляются по $C^{*}$-представлению, определяемому $K$-сферическими матричными коэффициентами для $\bar{\pi}_{n}^{\mathrm{p}}$. Это представление на самом деле является главным алгебраическим инструментом в [37].

Пусть снова $\pi_{0}$ - представление группы $G$ как в определении 1 . Во всех построениях выше главным строительным блоком для представлений $\bar{\pi}_{0}^{\mathrm{p}}$ является вполне положительное отображение $\Phi$ (см. теорему 37 ) с носителем $C^{*}(G)$ и со значениями в $B(L)$, продолжающееся на $C^{*}(\bar{G})$. Отображение $\Phi$ кодирует суммы в формуле (26). Продолжим $\Phi$ на $C^{*}(\bar{G})$, для характеристической функции замкнутого подмножества $C$ из $\bar{G}$ полагая

$$
\Phi\left(\chi_{C}\right)=\sum_{\theta \in C} P_{L} \pi(\theta) P_{L}
$$

Тогда $\Phi$ - вполне положительное отображение на $C^{*}(\bar{G})$ со значениями в $B(L)$; кроме того, $\Phi$ является *-сохраняющим мультипликативным представлением системы операторов (определение 13)

$$
\mathscr{O}(K, G)=\left[\mathbb{C}\left(\chi_{\sigma K} \mid \sigma \in G\right)\right] \cdot\left[\mathbb{C}\left(\chi_{\sigma K} \mid \sigma \in G\right)\right]^{*} \subseteq C^{*}(\bar{G})
$$

под *-сохраняющим мультипликативным свойством понимается следующее: для любых двух $K$-смежных классов $K \sigma_{1}, K \sigma_{2}$ в $\bar{G}$

$$
\Phi\left(\chi_{K \sigma_{1}}\right)^{*} \Phi\left(\chi_{K \sigma_{2}}\right)=\Phi\left(\chi_{\sigma_{1} K}\right) \Phi\left(\chi_{K \sigma_{2}}\right)=\Phi\left(\chi_{\sigma_{1} K \sigma_{2}}\right) .
$$


Мы докажем (см. предложение 40), что представление $\bar{\pi}_{0}^{\mathrm{p}}$ полностью восстанавливается по вполне положительному отображению $\Phi$.

Тогда $\Phi$ - “операторнозначный собственный вектор" для алгебры Гекке. Действительно, в силу свойства мультипликативности, обозначая операцию свертки на функциях на $\bar{G}$ через ·, получаем, что

$$
\Phi\left(\chi_{K \sigma_{1} K}\right) \Phi\left(\chi_{K \sigma_{2}}\right)=\Phi\left(\chi_{K \sigma_{1} K} \cdot \chi_{K \sigma_{2}}\right), \quad \sigma_{1}, \sigma_{2} \in G
$$

\section{3. Аксиомы для построения гильбертовых пространств Г-инвариантных векторов}

Пусть $\Gamma \subseteq G$ - почти нормальная подгруппа как во введении, удовлетворяющая условию $\left[\Gamma: \Gamma_{\sigma}\right]=\left[\Gamma: \Gamma_{\sigma^{-1}}\right]$ для всех $\sigma$ в $G$. Пусть $\pi-($ проективное) унитарное представление группы $G$ в унитарную группу $\mathscr{U}(H)$ гильбертова пространства $H$, обладающее свойствами (i) и (ii) из определения 1 . В частности, $\operatorname{dim}_{\{\pi(\Gamma)\}^{\prime \prime}} H$ - целое или $\infty$. Как замечено в предыдущем разделе, отсюда следует существование Г-блуждающего порождающего подпространства $L$ для $\left.\pi\right|_{\Gamma}$ (определение 13). Напомним, что это означает, что $L$ ортогонально к $\pi(\gamma) L$ при $\gamma \in \Gamma, \gamma \neq e$, и что $H=\bigvee_{\gamma \in \Gamma} \pi(\gamma) L$.

Мы строим гильбертовы пространства $H^{\Gamma_{0}} \quad \Gamma_{0}$-инвариантных векторов, где $\Gamma_{0} \in \mathscr{S}$. Такие пространства $H^{\Gamma_{0}}$ будут изометрично изоморфны $l^{2}\left(\Gamma_{0} \backslash \Gamma\right) \otimes L$ при $\Gamma_{0} \in \mathscr{S}$. Основная проблема, которую мы сначала рассмотрим в этом разделе, состоит в построении представления группы $G$ на объединении пространств $\Gamma_{0}$-инвариантных векторов.

Частные примеры, приведенные в предыдущем разделе, подсказывают, что возможный подход к этой проблеме состоит в нахождении вложения гильбертова пространства $H$ в большее векторное пространство $\mathscr{V}$ такое, что представление $\pi$ продолжается до представления $\pi_{\mathscr{V}}$ группы $G$ в группу линейных изоморфизмов пространства $\mathscr{V}$ и подпространство $H$ пространства $\mathscr{V}$ инвариантно относительно $\pi_{\mathscr{V}}$. Сначала мы поясняем построение в этом случае, затем выполняем построение на основе свойств (i) и (ii) из определения 1.

Мы будем работать с подгруппами $\Gamma_{0}$, которые сопряжены в $G$ с подгруппами из $\mathscr{S}$. Обозначим этот расширенный класс подгрупп группы $G$ через $\widetilde{\mathscr{S}}$.

Для $\Gamma_{0} \in \widetilde{\mathscr{S}}$ рассмотрим пространство $\mathscr{V} \Gamma_{0} \pi_{\mathscr{V}}\left(\Gamma_{0}\right)$-инвариантных векторов из $\mathscr{V}$. Тогда $\pi_{\mathscr{V}}$ обладает очевидным продолжением на

$$
\mathscr{V}_{\infty}=\bigvee_{\Gamma_{0} \in \widetilde{\mathscr{S}}} \mathscr{V}^{\Gamma_{0}}=\bigvee_{\Gamma_{0} \in \mathscr{S}} \mathscr{V}^{\Gamma_{0}} .
$$

Остается проблема нахождения $\pi_{\mathscr{V}}(G)$-инвариантного подпространства из $\mathscr{V}_{\infty}$, которое наделено $\pi_{\mathscr{V}}(G)$-инвариантным предгильбертовым скалярным произведением. Используя это скалярное произведение, мы определяем гильбертовы пространства $H^{\Gamma_{0}} \subseteq \mathscr{V}^{\Gamma_{0}}, \Gamma_{0} \in \widetilde{\mathscr{S}}$. Тогда $\pi_{\mathscr{V}}$ индуцирует унитарное представление группы $G$ на $\bigvee H^{\Gamma_{0}}$.

$$
\Gamma_{0} \in \widetilde{\mathscr{S}}
$$


Построение в этом разделе, конечно, похоже на другие построения в литературе (см., например, [6], [21]). Однако в теореме 22 мы используем это построение, чтобы ввести специфические *-представления алгебры Гекке, включающие выражения вида (26)). Они обобщаются в следующем разделе на случай, когда $\operatorname{dim}_{\pi(\Gamma)} H$ не целое и потому не существует Г-блуждающего порождающего подпространства.

В следующем определении мы вводим общий формализм, используемый для построения представления $\bar{\pi}$, начиная с продолжения заданного представления $\pi$ на большее векторное пространство, которое содержит векторы, инвариантные под действием подгрупп из $\mathscr{S}$. На практике, как будет сделано и ниже в этом разделе, непосредственно строятся гильбертовы пространства, соответствующие "виртуальным" $\Gamma_{0}$-инвариантным векторам, $\Gamma_{0} \in \mathscr{S}$.

ОПрЕДЕЛЕНИЕ 14 (формализм Г-инвариантных векторов). Пусть $\Gamma \subseteq G-$ как во введении, а $\pi$ - представление (возможно, проективное) группы $G$ в унитарную группу гильбертова пространства $H$. Сделаем следующие предположения.

(i) Существуют большее векторное пространство $\mathscr{V}$, содержащее $H$, и представление $\pi_{\mathscr{V}}$ группы $G$ в линейные изоморфизмы пространства $\mathscr{V}$ такие, что $\pi_{\mathscr{V}}(g)$ оставляет на месте $H$ и для всех $g \in G$ выполнено равенство $\left.\pi_{\mathscr{V}}(g)\right|_{H}=$ $\pi(g)$. Как и выше, для $\Gamma_{0} \in \widetilde{\mathscr{S}}$ обозначаем через $\mathscr{V}^{\Gamma_{0}}$ подпространство из $\mathscr{V}$, состоящее из векторов, неподвижных относительно действия группы $\Gamma_{0}$. Рассмотрим векторное пространство $\mathscr{V}_{\infty}$, введенное в $(28)$.

(ii) Существуют всюду плотное $\pi(G)$-инвариантное подпространство $\mathscr{D}_{\mathscr{V}} \subseteq H$ и комплекснозначная билинейная форма $\langle\cdot, \cdot\rangle_{\infty}$ на

$$
\mathscr{V}_{\infty} \times\left(\mathscr{V}_{\infty} \vee \mathscr{D}_{\mathscr{V}}\right)
$$

со следующими свойствами:

(ii.1) ограничение $\langle\cdot, \cdot\rangle_{\infty}$ на $\mathscr{V}_{\infty} \times \mathscr{V}_{\infty}$ является положительно определенным предгильбертовым скалярным произведением;

(ii.2) для каждых $\Gamma_{0} \in \widetilde{\mathscr{S}}$ и $v \in \mathscr{V} \Gamma_{0}$ линейное отображение на $\mathscr{D} \mathscr{V}$, определяемое ограничением линейной формы $\langle v, \cdot\rangle_{\infty}$ на $\mathscr{D} \mathscr{V}$, является $\Gamma_{0}$-инвариантным;

(ii.3) $\langle\cdot, \cdot\rangle_{\infty}$ является $\pi_{\mathscr{V}}(G)$-инвариантным:

$$
\left\langle\pi_{\mathscr{V}}(g) v_{1}, \pi_{\mathscr{V}}(g) v_{2}\right\rangle_{\infty}=\left\langle v_{1}, v_{2}\right\rangle_{\infty}, \quad g \in G, \quad v_{1} \in \mathscr{V}_{\infty}, \quad v_{2} \in \mathscr{V}_{\infty} \vee \mathscr{D}_{\mathscr{V}}
$$

Если выполняются вышеперечисленные предположения, обозначим через $H^{\Gamma_{0}}$ гильбертово пространство пополнения $\mathscr{V}^{\Gamma_{0}}$ относительно скалярного произведения $\langle\cdot, \cdot\rangle_{\infty}$, и пусть $\bar{H}^{\mathrm{p}}$ - гильбертово пространство пополнения пространства $\mathscr{V}_{\infty}$ относительно этого скалярного произведения.

Следующая лемма является очевидным следствием предположений из определения 14.

Лемма 15. Предположим, что выполняются условия определения 14. Тогда ограничение $\pi_{\mathscr{V}}$ на $\mathscr{V}_{\infty}$ продолжается до унитарного представления $\bar{\pi}^{\mathrm{p}}$ группы $G$ в унитарную группу пространства $\bar{H}^{\mathrm{p}}$. Более того, $\bar{\pi}^{\mathrm{p}}$ изометрически отображает $H^{\Gamma_{0}}$ на $H^{\sigma \Gamma_{0} \sigma^{-1}} \partial л я \Gamma_{0} \in \mathscr{S}, \sigma \in G$. 
Если $\Gamma_{1} \subseteq \Gamma_{0}, \Gamma_{1}, \Gamma_{0} \in \widetilde{\mathscr{S}}$, то включение $H^{\Gamma_{0}}$ в $H^{\Gamma_{1}}$ изометрическое по построению. Ортогональная проекиия из $H^{\Gamma_{1}}$ на $H^{\Gamma_{0}}$ получается усреднением по смежным классам группы $\Gamma_{0}$ в $\Gamma_{1}$.

Если исходное представление $\pi$ проективно (см., например, [5] и ссылки там) с коциклом $\epsilon \in H^{2}(G, \mathbb{T})$, то при условии, что продолжение $\pi_{\mathscr{V}}$ имеет тот же коцикл, проведенное выше построение все еще работает.

Впоследствии мы будем работать одновременно с представлениями группы $G$ и пополнения Шлихтинга $\bar{G}[48]$. Поэтому мы вводим универсальную $C^{*}$-алгебру, содержащую и $C^{*}(G)$, и $C^{*}(\bar{G})$ как $C^{*}$-подалгебры.

ОПредЕЛЕНиЕ 16 . Пусть $G$ и $\bar{G}$ - такие, как выше, и пусть $\mathscr{A}(G, \bar{G})$ - факторалгебра универсального скрещенного произведения $C^{*}$-алгебр $C^{*}\left(G \rtimes C^{*}(\bar{G})\right)$, где $G$ действует сопряжением на $C^{*}(\bar{G})$, по замкнутому по норме идеалу, порожденному соотношениями вида

$$
g \chi_{K_{0}}=\chi_{g K_{0}}, \quad g \in G, \quad K_{0}=\bar{\Gamma}_{0}, \quad \Gamma_{0} \in \mathscr{S} .
$$

Здесь $\chi_{g K_{0}}$ обозначает характеристическую функцию смежного класса

$$
g K_{0}=\overline{g \Gamma_{0}},
$$

где операция замыкания рассматривается в $\bar{G}$.

Тогда $\mathscr{A}(G, \bar{G})$ является замыканием по норме оболочки

$$
\operatorname{Sp}\left\{g \chi_{K_{0}} \mid g \in G, K_{0}=\bar{\Gamma}_{0}, \Gamma_{0} \in \mathscr{S}\right\}
$$

Пусть задан коцикл $\epsilon \in H^{2}(G, \mathbb{T})$, который продолжается на $H^{2}(\bar{G}, \mathbb{T})$. Тогда, работая со скрещенными произведениями с коциклом, получаем аналогичную $C^{*}$-алгебру, которую обозначим через $\mathscr{A}_{\epsilon}(G, \bar{G})$.

Используя два предыдущих определения, докажем, что представление $\bar{\pi}^{\mathrm{p}}$ одновременно продолжается на $G$ и на $\bar{G}$.

ПреДЛОжЕНИЕ 17. Пусть $\pi$ - представление из определения 14. Тогда соответствующее представление $\bar{\pi}^{\mathrm{P}}$ из леммы 15 продолжается до представления $C^{*}$-алгебры $\mathscr{A}_{\epsilon}(G, \bar{G})$ в $B\left(\bar{H}^{\mathrm{p}}\right)$ (которое мы также будем обозначать через $\overline{\pi^{\mathrm{p}}}$ ).

ДокАЗАтельство. Пусть $\Gamma_{0}$ - подгруппа группы $G$, принадлежащая классу $\widetilde{\mathscr{S}}$. Пусть $K_{0}=\bar{\Gamma}_{0}$, где замыкание берется в топологии группы $\bar{G}$. Пусть $P_{H^{\Gamma_{0}}}$ - ортогональная проекция из $\bar{H}^{\mathrm{p}}$ на гильбертово пространство $H^{\Gamma_{0}}$. Продолженное представление $\bar{\pi}^{\mathrm{p}}$ строится отображением $\frac{1}{\mu\left(K_{0}\right)} \chi_{K_{0}}$. Нормировка необходима, так как в $C^{*}(\bar{G})$ свертка с подгруппой $K_{0}$ группы $K$ является нетривиальным скалярным кратным проекции, поскольку $\left(\chi_{K_{0}}\right)^{2}=\mu\left(K_{0}\right) \chi_{K_{0}}$. Элементы из $G$ представлены унитарными операторами на $\bar{H}^{\mathrm{p}}$ с помощью представления $\bar{\pi}^{\mathrm{p}}$ из леммы 15. При таком выборе все соотношения, определяющие универсальную $C^{*}$-алгебру $\mathscr{A}_{\epsilon}(G, \bar{G})$, очевидно, выполняются. Предложение доказано. 
Для заданного представления $\pi$ группы $G$ такого, что $\left.\pi\right|_{\Gamma}$ допускает Г-блуждающее порождающее подпространство, мы построим представление из определения 14. Гильбертовы пространства $H^{\Gamma_{0}}$-инвариантных векторов мы построим непосредственно, без построения пространства $\mathscr{V}$ из определения 14.

Лемма 18. Пусть $\pi$ - унитарное представление группъ $G$, обладающее свойствами (i) и (ii) из определения 1. В частности, $\left.\pi\right|_{\Gamma}-$ иелое кратное левого регулярного представления $\lambda_{\Gamma}$. Мы используем обозначения из определения 1. Тогда справедливы следующие утверждения.

(i) Для всех $\Gamma_{0} \in \mathscr{S}$ u $g \in G$ сумма по смежному классу $\Gamma_{0} g$

$$
\sum_{\theta \in \Gamma_{0} g} P_{L} \pi(\theta) P_{L}
$$

сходится в $B(L)$.

(ii) Подпространство

$$
\mathscr{D}_{L, \pi}=\left\{h \in H \mid \sum_{\gamma \in \Gamma_{0}} P_{L} \pi(\gamma) h \text { сходится для всех } \Gamma_{0} \in \mathscr{S}\right\}
$$

является всюду плотным $\pi(G)$-инвариантным подпространством из $H$, содержащим $L$.

ДокАЗАТЕЛЬство. (i) Чтобы доказать утверждение (i), заметим, что достаточно доказать его для $\Gamma_{0}=\Gamma$, так как $\left.\pi\right|_{\Gamma_{0}}$ остается целым кратным левого регулярного представления $\lambda_{\Gamma_{0}}$ группы $\Gamma_{0}$. Если просуммировать по двойному смежному классу Г $\sigma$ Г, то получится в точности оператор Гекке, ассоциированный с двойным смежным классом, действующий на пространстве $L$, которое унитарно эквивалентно пространству Г-инвариантных векторов.

Если представление $\pi$ таково, как в примере 28 из раздела 4, то сумма (29) совпадает с представлением в унитарном представлении Купмана кусочно биективного преобразования $\dot{\widehat{\Gamma g}}$, введенного в [41; лемма 4, (i)]. Сумма сходится, так как мы добавляем частичные изометрии, соответствующие преобразованиям с попарно непересекающимися областями. Следовательно [41], оператор, ассоциированный с кусочно биективным преобразованием $\dot{\widehat{\Gamma g}}$, является конечной суммой (мощности $\left.\left[\Gamma: \Gamma_{g}\right]\right)$ частичных изометрий с ортогональными исходными пространствами. Это рассуждение дословно переносится на наш случай благодаря предположению (ii) в определении 1. Ключевое свойство, в силу которого рассуждение работает, - это то, что $\pi(\sigma) L$ является $\sigma \Gamma \sigma^{-1}$-блуждающим подпространством.

(ii) Заметим, что для того, чтобы доказать всюду плотность, достаточно предположить, что Г-эквивариантным образом выполняется $H=\ell^{2}(\Gamma)$. В этом случае область $\mathscr{D}_{\mathscr{V}}$ - это просто $\ell^{1}(\Gamma) \cap \ell^{2}(\Gamma)$. Теперь $\pi(G)$-инвариантность пространства $H$ следует из утверждения (i).

Лемма доказана.

ОПРедЕЛЕНиЕ 19. Мы используем обозначения и определения, введенные выше. Пусть $\Gamma_{0}-$ подгруппа из $\mathscr{S}$. Предположим, что Г имеет разложение 
на смежные классы по $\Gamma_{0}$ вида $\Gamma=\bigcup \Gamma_{0} r_{j}$, где $r_{j}$ - представители смежных классов для $\Gamma_{0}$. Пусть $L^{\Gamma_{0}}-$ подпространство пространства $H$, полученное суммированием следующих ортогональных подпространств пространства $H$ :

$$
L^{\Gamma_{0}}=\sum \pi\left(r_{j}\right) L
$$

Обозначим ортогональную проекцию из $H$ на $L^{\Gamma_{0}}$ через $P_{L^{\Gamma_{0}}}$.

Определим гильбертово пространство $H^{\Gamma_{0}}=\mathscr{V}^{\Gamma_{0}}$ как пространство формальных сумм

$$
\left\{\sum_{\gamma_{0} \in \Gamma_{0}} \pi\left(\gamma_{0}\right) h \mid h \in \mathscr{D}_{L, \pi}\right\}
$$

при отождествлении

$$
\sum_{\gamma_{0} \in \Gamma_{0}} \pi\left(\gamma_{0}\right) h=\sum_{\gamma_{0} \in \Gamma_{0}} \pi\left(\gamma_{0}\right) l_{0},
$$

если $h \in \mathscr{D}_{L, \pi}$ и $l_{0}-$ вектор из $L^{\Gamma_{0}}$, заданный формулой

$$
l_{0}=\sum_{\gamma_{0} \in \Gamma_{0}} P_{L^{\Gamma_{0}}}\left(\pi\left(\gamma_{0}\right) h\right) .
$$

Бесконечная сумма в (34) сходится, так как $h$ принадлежит $\mathscr{D}_{L, \pi}$.

Условие (33) соответствует тому, что сумма по Г инвариантна относительно замены переменной суммирования $\gamma$ на $\gamma \gamma_{0}$ для фиксированного $\gamma_{0}$ из $\Gamma_{0}$. Это условие должно обязательно выполняться, если вектор $h$ является суммой сдвигов векторов из $L^{\Gamma_{0}}$ на элементы из $\Gamma_{0}$. Используя вышеупомянутое построение, мы можем ввести унитарное представление группы $\bar{G}$, действующее на векторах, инвариантных относительно подгрупп из $\mathscr{S}$.

ПредлОЖенИЕ 20. Положительно определенное предгилъбертово скалярное произведение $\langle\cdot, \cdot\rangle_{\infty}$ на $\mathscr{V}_{\infty}=\bigvee_{\Gamma_{0} \in \mathscr{S}} H^{\Gamma_{0}}$ определяется для $\Gamma_{0} \in \mathscr{S}, l_{1} \in$ $\mathscr{D}_{\Gamma, \pi}, l_{2} \in L^{\Gamma_{0}}$ формулой

$$
\left\langle\sum_{\gamma_{0} \in \Gamma_{0}} \pi\left(\gamma_{0}\right) l_{1}, \sum_{\gamma_{0}^{\prime} \in \Gamma_{0}} \pi\left(\gamma_{0}^{\prime}\right) l_{2}\right\rangle_{\infty}=\frac{1}{\left[\Gamma: \Gamma_{0}\right]}\left\langle\sum_{\gamma_{0} \in \Gamma_{0}} \pi\left(\gamma_{0}\right) l_{1}, l_{2}\right\rangle
$$

В правой части этого равенства используется скалярное произведение на $H$. Ясно, что $\mathscr{V}^{\Gamma_{0}}$ изометрически вкладьвается в $\mathscr{V}^{\Gamma_{1}}$ для $\Gamma_{1} \subseteq \Gamma_{0}$. Пусть $\bar{H}^{\mathrm{p}}$ гилъбертово пространство пополнения пространства $\mathscr{V}_{\infty}$ относительно скалярного произведения $\langle\cdot, \cdot\rangle_{\infty}$.

Тогда унитарное представление $\pi$ определяет унитарное представление $\bar{\pi}^{\mathrm{p}}$ в унитарную группу пространства $\bar{H}^{\mathrm{p}}$, обладающее свойствами из леммы 15.

Прежде чем доказывать это предложение, заметим, что формула (35), эквивалентная формуле (36) ниже, является обобщением формулы скалярного произведения Петерссона [34].

Действительно, придерживаясь обозначений из введения, рассмотрим случай двух автоморфных форм веса $n \in \mathbb{N}$, которые поэтому являются Г-инвариантными векторами, как выше, для представления $\pi_{n}$. Чтобы получить 
скалярное произведение двух автоморфных форм, одну из них умножают на характеристическую функцию $\chi_{F}$ фундаментальной области и затем используют обычное скалярное произведение из $L^{2}\left(\mathbb{H}, \nu_{n}\right)$, которое продолжает скалярное произведение на $H_{n}$. Именно это осуществляется в следующей формуле путем замены оператора $M_{\chi_{F}}$ (из формулы $(26)$ ) на проекцию $P_{L}$, которая имеет схожие с $M_{\chi_{F}}$ свойства.

Формулу (35) можно переписать в эквивалентном виде, аналогичном формуле скалярного произведения Петерссона. Для $h_{1}, h_{2} \in \mathscr{D}_{L, \pi}$ положим

$$
l_{i}=\sum_{\gamma \in \Gamma} P_{L} \pi(\gamma) h_{i}, \quad i=1,2 .
$$

Используя отождествление (33), получаем, что формула (35) эквивалентна следующей:

$$
\left\langle\sum_{\gamma \in \Gamma} \pi(\gamma) h_{1}, \sum_{\gamma^{\prime} \in \Gamma} \pi\left(\gamma^{\prime}\right) h_{2}\right\rangle_{\infty}=\left\langle P_{L}\left(\sum_{\gamma \in \Gamma} \pi(\gamma)\right) h_{1}, \sum_{\gamma^{\prime} \in \Gamma} \pi\left(\gamma^{\prime}\right) h_{2}\right\rangle .
$$

Это равно

$$
\left\langle P_{L}\left(\sum_{\gamma \in \Gamma} \pi(\gamma)\right) h_{1}, P_{L}\left(\sum_{\gamma^{\prime} \in \Gamma} \pi\left(\gamma^{\prime}\right) h_{2}\right)\right\rangle=\left\langle l_{1}, l_{2}\right\rangle .
$$

Для более общих подгрупп $\Gamma_{0} \in \mathscr{S}$ формула (36) скалярного произведения аналогична, с той разницей, что вместо $P_{L}$ используется проекция $P_{L^{\Gamma_{0}}}$ на $\Gamma_{0}$-блуждающее порождающее подпространство $\pi$.

ДОКАЗАТЕЛЬСТво ПРЕДЛОЖЕНИЯ 20. Пусть $\Gamma_{0}, \Gamma_{1}$ - две подгруппы из $\mathscr{S}$ такие, что $\Gamma_{1} \subseteq \Gamma_{0}$. Сначала разобьем $\Gamma_{0}$ на смежные классы по $\Gamma_{1}$. Используя представителей смежных классов, разобьем сумму в формуле (32) для векторов из $\mathscr{V}^{\Gamma_{0}}$ на $\left[\Gamma_{0}: \Gamma_{1}\right]$ векторов, которые все принадлежат $\mathscr{V}^{\Gamma_{1}}$. Тогда $\mathscr{V} \Gamma_{0}$ вложено в $\mathscr{V}^{\Gamma_{1}}$. Действительно, если $\Gamma_{0}=\bigcup r_{j} \Gamma_{1}$, то вложение реализуется следующим образом: если

$$
\eta=\sum_{\gamma_{0} \in \Gamma_{0}} \pi\left(\gamma_{0}\right) l_{0}, \quad l_{0} \in L^{\Gamma_{0}},
$$

- вектор общего положения из $\mathscr{V} \Gamma_{0}$, то мы отождествляем $\eta$ со следующим элементом векторного пространства $\mathscr{V}^{\Gamma_{1}}$ :

$$
\eta_{1}=\sum_{j} \sum_{\gamma_{1} \in \Gamma_{1}} \pi\left(\gamma_{1}\right) l_{0}=\sum_{\gamma_{1} \in \Gamma_{1}} \pi\left(\gamma_{1}\right)\left[\sum_{j} \pi\left(r_{j}\right) l_{0}\right] \in \mathscr{V}^{\Gamma_{1}}
$$

Вложение $\mathscr{V}^{\Gamma_{0}} \subseteq \mathscr{V}^{\Gamma_{1}}$ изометрическое. Действительно, для $l_{0}$ из $L^{\Gamma_{0}}$ квадрат нормы вектора $\eta \in H^{\Gamma_{0}}$, введенного в (37), определяется в соответствии с формулой (35) и равен

$$
\frac{1}{\left[\Gamma: \Gamma_{0}\right]}\left\langle l_{0}, l_{0}\right\rangle
$$

где скалярное произведение вычисляется в $H$. 
С другой стороны, в соответствии с той же формулой норма вектора $\eta_{1}$ из $\mathscr{V}^{\Gamma_{1}}$ равна

$$
\frac{1}{\left[\Gamma: \Gamma_{1}\right]}\left\langle\left[\sum_{j} \pi\left(r_{j}\right) l_{0}\right],\left[\sum_{k} \pi\left(r_{k}\right) l_{0}\right]\right\rangle .
$$

Множество $\left\{r_{j}\right\}$ имеет мощность $\left[\Gamma_{0}: \Gamma_{1}\right]$. Более того, векторы $\pi\left(r_{j}\right) l_{0}$ попарно ортогональны. Отсюда следует, что квадрат нормы вектора $\eta_{1}$ равен

$$
\frac{1}{\left[\Gamma: \Gamma_{1}\right]}\left[\Gamma_{0}: \Gamma_{1}\right]\left\langle l_{0}, l_{0}\right\rangle=\left[\Gamma: \Gamma_{1}\right]\left\langle l_{0}, l_{0}\right\rangle,
$$

а значит, вложение $\mathscr{V}^{\Gamma_{0}}$ в $\mathscr{V}^{\Gamma_{1}}$ изометрическое.

Представление $\pi_{\mathscr{V}}$ определяется следующим образом. Пусть $g \in G, \Gamma_{0} \in \mathscr{S}$, $l \in L ;$ рассмотрим вектор

$$
\eta=\sum_{\gamma_{0} \in \Gamma_{0}} \pi\left(\gamma_{0}\right) l \in \mathscr{V}^{\Gamma_{0}} .
$$

Представим смежный класс $g \Gamma_{0}$ как объединение

$$
g \Gamma_{0}=\bigcup_{j} \Gamma_{0}^{j} y_{j}
$$

попарно непересекающихся смежных классов меньших подгрупп $\Gamma_{0}^{j}$ из $\mathscr{S}$ таких, что

$$
g \Gamma_{0}^{j} g^{-1}=\Gamma_{1}^{j} \subseteq \Gamma, \quad \Gamma_{1}^{j} \in \mathscr{S} .
$$

Это всегда можно сделать, рассматривая смежные классы $\Gamma_{0}$ по подгруппам из $\Gamma_{0} \cap \Gamma_{g^{-1}}$. Тогда мы определяем

$$
\pi_{\mathscr{V}}(g) \eta=\sum_{j} \sum_{\gamma_{j} \in \Gamma_{1}^{j}} \pi\left(\gamma_{j}\right)\left(\pi\left(g y_{j}\right) l\right) .
$$

В силу предположений на область (30) получаем, что $\pi_{\mathscr{V}}(g) \eta$ лежит в $\mathscr{V}_{\infty}$. Это верно потому, что $\pi_{\mathscr{V}}(g)$ отображает $\mathscr{V}_{0}^{\Gamma_{0}^{j} \cap \Gamma_{g^{-1}}}$ на $\mathscr{V} \Gamma_{1}^{j} \cap \Gamma_{g}$. Поскольку для всех $g \in G$ индексы подгрупп $\Gamma_{g^{-1}}$ и $\Gamma_{g}$ равны, определение скалярного произведения доказывает, что $\pi_{\mathscr{V}}$ изометрически отображает $\mathscr{V}_{0}$ в $\mathscr{V}_{\infty}$. Очевидно, что если $g \in G$ и $\Gamma_{0} \in \mathscr{S}$, то $\mathscr{V}^{g \Gamma_{0} g^{-1}}$ содержится в $\mathscr{V}^{g \Gamma_{0} g^{-1} \cap \Gamma_{0}}$. Но $g \Gamma_{0} g^{-1} \cap \Gamma_{0}$ является подгруппой из $\mathscr{S}$, поэтому мы получаем альтернативную формулу $\mathscr{V}_{\infty}=\bigvee_{\Gamma_{0} \in \widetilde{\mathscr{S}}} H^{\Gamma_{0}}$

Следовательно, получено унитарное представление $\bar{\pi}^{\mathrm{p}}$ в унитарную группу гильбертова пространства $\bar{H}^{\mathrm{p}}$ из определения 14. Предложение доказано.

Напомним, что для $\Gamma_{0} \in \mathscr{S}$ и $\sigma \in G$ мы обозначаем

$$
\left(\Gamma_{0}\right)_{\sigma}=\sigma \Gamma_{0} \sigma^{-1} \cap \Gamma_{0} .
$$

Индекс $\left[\Gamma_{0}:\left(\Gamma_{0}\right)_{\sigma}\right]$ встретится в дальнейших вычислениях.

Для $\Gamma_{0} \in \mathscr{S}$ пусть $K_{0}=\bar{\Gamma}_{0}$ - замыкание группы $\Gamma_{0}$ в проконечном пополнении $K$ группы $Г$. Следующее утверждение описывает явный вид матричного 
представления образа при представлении $\bar{\pi}^{\mathrm{p}}$ оператора свертки с характеристической функцией двойного смежного класса $K_{0} \sigma K_{0}$. Это, очевидно, оператор Гекке на $\Gamma_{0}$-инвариантных векторах, ассоциированный с двойным смежным классом $\Gamma_{0} \sigma \Gamma_{0}$ и нормированный константой. Мы получим сначала точную константу нормировки, требуемую для операторов Гекке. Полученный нормирующий множитель является индексом подгруппы $\left(\Gamma_{0}\right)_{\sigma}$ в $\Gamma_{0}$. Он нужен потому, что в $C^{*}$-алгебре $C^{*}(\bar{G}, G)$, если $K_{0}$ - подгруппа из $K$, то оператор свертки с $\chi_{K_{0} \sigma K_{0}}$ является скалярным кратным с множителем $\left[K_{0}:\left(K_{0}\right)_{\sigma}\right]$ упорядоченного произведения операторов свертки с $\chi_{K_{0}}, \sigma$ и $\chi_{K_{0}}$.

Лемма 21. Во введенных выше обозначениях для каждого $\sigma \in G$ и каждой подгруппы $K_{0}$ как выше имеем

$$
\bar{\pi}^{\mathrm{p}}\left(\chi_{K_{0} \sigma K_{0}}\right)=\left[\Gamma:\left(\Gamma_{0}\right)_{\sigma}\right]\left(\bar{\pi}^{\mathrm{p}}\left(\chi_{K_{0}}\right) \bar{\pi}^{\mathrm{p}}(\sigma) \bar{\pi}^{\mathrm{p}}\left(\chi_{K_{0}}\right)\right) .
$$

ДокАзАтельство. Мы работаем в универсальной алгебре $\mathscr{A}=C^{*}(G, \bar{G})$. Для $\sigma \in \bar{G}$ обозначим свертку с $\sigma$ через $L_{\sigma}$. Обозначим оператор свертки с непрерывной функцией $f$ на $\bar{G}$ через $L(f)$. Ясно, что для каждого измеримого подмножества $A$ из $\bar{G}$

$$
L\left(\chi_{A}\right) L_{\sigma}=L\left(\chi_{A \sigma}\right), \quad L_{\sigma} L\left(\chi_{A}\right)=L\left(\chi_{\sigma A}\right), \quad \sigma \in G .
$$

Тогда очевидно, что

$$
L(f)=\int_{\bar{G}} f(g) L_{g} d g
$$

и

$$
\left(L\left(\chi_{K_{0}}\right)\right)^{2}=\nu\left(K_{0}\right) L\left(\chi_{K_{0}}\right) .
$$

В частности, $\nu\left(K_{0}\right)^{-1} L\left(\chi_{K_{0}}\right)-$ проекция.

То же рассуждение дает, что если $K_{0}, K_{1}$ - подгруппы как выше, а $K_{1}$ подгруппа из $K_{0}$, то

$$
L\left(\chi_{K_{1}}\right) L\left(\chi_{K_{0}}\right)=L\left(\chi_{K_{0}}\right) L\left(\chi_{K_{1}}\right)=\nu\left(K_{1}\right) L\left(\chi_{K_{0}}\right) .
$$

Разложим $K_{0}$ в объединение правых смежных классов для подгрупп $\left(K_{0}\right)_{\sigma}=$ $K_{0} \cap \sigma K_{0} \sigma^{-1}$ с представителями смежных классов $s_{i} \in K_{0}, i=1,2, \ldots$, $\left[K_{0}:\left(K_{0}\right)_{\sigma}\right]$. Это те же представители смежных классов, что и для $\left(\Gamma_{0}\right)_{\sigma}$ в $\Gamma_{0}$. Используя формулу (42), получаем

$$
\begin{aligned}
L\left(\chi_{K_{0}}\right) L_{\sigma} L\left(\chi_{K_{0}}\right) & =\sum_{i} L\left(s_{i} \chi_{\left(K_{0}\right)_{\sigma}}\right) L_{\sigma} L\left(\chi_{K_{0}}\right)=\sum_{i} L\left(s_{i} \sigma\right) L\left(\chi_{\left(K_{0}\right)_{\sigma-1}}\right) L\left(\chi_{K_{0}}\right) \\
& =\nu\left(\left(K_{0}\right)_{\sigma^{-1}}\right) \sum_{i} L\left(s_{i} \sigma\right) L\left(\chi_{K_{0}}\right)=\nu\left(\left(K_{0}\right)_{\sigma^{-1}}\right) L\left(\chi_{K_{0} \sigma K_{0}}\right) .
\end{aligned}
$$

Лемма доказана.

Как было отмечено выше, гильбертовы пространства $H^{\Gamma_{0}}, \Gamma_{0} \in \mathscr{S}$, изометрически изоморфны $l^{2}\left(\Gamma_{0} \backslash \Gamma\right) \otimes L$, где скалярное произведение на $l^{2}\left(\Gamma_{0} \backslash \Gamma\right)$ выбирается так, чтобы вложения $l^{2}\left(\Gamma_{0} \backslash \Gamma\right) \subseteq l^{2}\left(\Gamma_{1} \backslash \Gamma_{0}\right)$ были изометрическими 
для всех $\Gamma_{0} \subseteq \Gamma_{1}$. Оказывается, что элементы матриц, представляющих операторы Гекке, являются суммами вида (29). Лемма 31 ясно показывает, что после нормировки формула классического оператор Гекке, соответствующего сумме по смежным классам, - это

$$
\left[\Gamma: \Gamma_{\sigma}\right] P_{H^{\Gamma_{0}}} \bar{\pi}^{\mathrm{p}}(\sigma) P_{H^{\Gamma_{0}}}, \quad \sigma \in G, \quad \Gamma_{0} \in \mathscr{S} .
$$

Теорема 22. Мы используем обозначения и определения, введенные выше в этом разделе. Зафиксируем подгруппу $\Gamma_{0}$ из $\mathscr{S}$. Выберем семейство $\left(s_{i}\right)$ представителей правых смежных классов для $\Gamma_{0} \subseteq \Gamma$. Рассмотрим, как и в (31), гилъбертово пространство

$$
L^{\Gamma_{0}}=\bigoplus_{i=1}^{\left[\Gamma: \Gamma_{0}\right]} \pi\left(s_{i}\right) L
$$

Норма гилъбертова пространства на $L^{\Gamma_{0}}$ нормирована так, что вложение $L$ в $L^{\Gamma_{0}}$, определенное соответствием

$$
l \in L \rightarrow \bigoplus_{i=1}^{\left[\Gamma: \Gamma_{0}\right]} \pi\left(s_{i}\right) l \in L^{\Gamma_{0}}=\bigoplus_{i=1}^{\left[\Gamma: \Gamma_{0}\right]} \pi\left(s_{i}\right) L,
$$

изометрическое. Пространство $L^{\Gamma_{0}}$ очевидным образом отождествляется с подпространством пространства $H$. В этом случае пространство $L^{\Gamma_{0}}$ наделено ненормированным скалярным произведением, унаследованным из $H$. Пусть $P_{L^{\Gamma_{0}}}$ - ортогональная проекиия из $H$ на $L^{\Gamma_{0}}$.

Тогда для $\sigma \in G$ оператор Гекке $\left[\Gamma: \Gamma_{\sigma}\right] P_{H^{\Gamma_{0}}} \bar{\pi}^{\mathrm{P}}(\sigma) P_{H^{\Gamma_{0}}}$ унитарно эквивалентен ограниченному оператору

$$
A\left(\Gamma_{0} \sigma \Gamma_{0}\right)=\sum_{\theta \in \Gamma_{0} \sigma \Gamma_{0}} P_{L^{\Gamma_{0}}} \pi(\theta) P_{L^{\Gamma_{0}}} .
$$

ДокАЗАТЕльство. Для заданных $\Gamma_{0} \in \mathscr{S}$ и выбора представителей смежных классов,

$$
\Gamma=\bigcup \Gamma_{0} s_{i}
$$

построим унитарный оператор $W^{\Gamma_{0}}$ из $L^{\Gamma_{0}}=\bigoplus \pi\left(s_{i}\right) L$ в $H^{\Gamma_{0}}$ следующим образом. Для векторов $l_{i} \in L$ определим следующую изометрию:

$$
W^{\Gamma_{0}}\left(\bigoplus \pi\left(s_{i}\right) l_{i}\right)=\sum_{i} \sum_{\gamma \in \Gamma_{0}} \pi\left(\gamma_{0}\right) \pi\left(s_{i}\right) l_{i}
$$

Докажем, что для $\sigma \in G$ следующая диаграмма коммутативна:

$$
\begin{aligned}
& H^{\Gamma_{0}} \stackrel{W^{\Gamma_{0}}}{\longleftarrow} \bigoplus \pi\left(s_{i}\right) L \\
& {\left[\Gamma_{0}:\left(\Gamma_{0}\right)_{\sigma}\right] P_{H \Gamma_{0}} \bar{\pi}^{\mathrm{p}}(\sigma) P_{H^{\Gamma_{0}}} \downarrow \backslash \sum_{\theta \in \Gamma_{0} \sigma \Gamma_{0}} P_{L} \Gamma_{0} \pi(\theta) P_{L \Gamma_{0}}} \\
& H^{\Gamma_{0}} \stackrel{W^{\Gamma_{0}}}{\longleftarrow} \bigoplus \pi\left(s_{i}\right) L
\end{aligned}
$$


Для этого воспользуемся формулой для унитарных операторов $\bar{\pi}^{\mathrm{p}}(\theta), \theta \in G$, определенных в доказательстве предложения 20.

Достаточно проверить коммутативность этой диаграммы в случае $\Gamma=\Gamma_{0}$; случаи, соответствующие другим подгруппам $\Gamma_{0} \in \mathscr{S}$, являются следствием. Рассмотрим вектор $l \in L$. Имеем

$$
W^{\Gamma} l=\sum_{\gamma \in \Gamma} \pi(\gamma) l \in \mathscr{V}^{\Gamma}
$$

Если разложение группы $\Gamma$ на правые смежные классы по $\Gamma_{\sigma^{-1}}$ есть $\Gamma=$ $\bigcup \Gamma_{\sigma^{-1}} r_{j}$, то

$$
W^{\Gamma} l=\sum_{j} \sum_{\gamma \in \Gamma_{\sigma^{-1}}} \pi(\gamma) \pi\left(r_{j}\right) l .
$$

Тогда, применяя $\bar{\pi}^{\mathrm{p}}(\sigma)$, получим

$$
\sum_{j} \sum_{\gamma_{1} \in \Gamma_{\sigma}} \pi\left(\gamma_{1}\right) \pi\left(\sigma r_{j}\right) l
$$

Проектируя на $H^{\Gamma}$, отсюда получаем

$$
\frac{1}{\left[\Gamma: \Gamma_{\sigma}\right]} \sum_{j} \sum_{\gamma \in \Gamma} \pi(\gamma) \pi\left(\sigma r_{j}\right) l,
$$

что равно

$$
\frac{1}{\left[\Gamma: \Gamma_{\sigma}\right]} \sum_{\theta \in \Gamma \sigma \Gamma} \pi(\theta) l .
$$

С другой стороны, сумма

$$
\sum_{\theta \in \Gamma \sigma \Gamma} P_{L} \pi(\theta) P_{L}
$$

примененная к вектору $l$, дает

$$
\sum_{\theta \in \Gamma \sigma \Gamma} P_{L} \pi(\theta) l=\sum_{j} \sum_{\gamma \in \Gamma} P_{L} \pi(\gamma) \pi\left(\sigma r_{j}\right) l .
$$

Применим изометрию $W^{\Gamma}$ к этому вектору. Воспользуемся отождествлениями, принятыми в структуре пространства $H^{\Gamma}$ (см. формулу (33) в определении 19). Тогда вышеупомянутая сумма соответствует вектору

$$
\sum_{r_{j}} \sum_{\gamma \in \Gamma} \pi\left(\gamma \sigma r_{j}\right) l=\sum_{\theta \in \Gamma \sigma \Gamma} \pi(\theta) l .
$$

Теорема доказана.

Ниже мы опишем несложные включения между пространствами векторов, инвариантных относительно подгрупп из $\mathscr{S}$. 
Лемма 23. Рассмотрим семейство гильбертовых пространств $l^{2}\left(\Gamma_{0} \backslash \Gamma\right)$, $\Gamma_{0} \in \mathscr{S}$. На этом семействе гилъбертовых пространств скалярное произведение нормировано так, что вложения $l^{2}\left(\Gamma_{0} \backslash \Gamma\right) \subseteq l^{2}\left(\Gamma_{1} \backslash \Gamma\right)$ изометрические для всех $\Gamma_{0} \subseteq \Gamma_{1}$. Тогда выполняется следующее.

(i) Гильбертовы пространства $H^{\Gamma_{0}}$ изометрически изоморфны $l^{2}\left(\Gamma_{0} \backslash \Gamma\right) \otimes L$ для всех $\Gamma_{0} \in \mathscr{S}$, при этом имеют место включение

$$
H^{\Gamma_{0}} \subseteq H^{\Gamma_{1}}
$$

полученное для $\Gamma_{1} \subseteq \Gamma_{0}$ с помощъю взятия тензорного произведения $с L$, и изометрическое включение

$$
l^{2}\left(\Gamma_{0} \backslash \Gamma\right) \subseteq l^{2}\left(\Gamma_{1} \backslash \Gamma\right) .
$$

(ii) Пусть $\pi$ - представление, обладающее свойствами (i), (ii) из определения 1. Тогда гильбертово пространство $\bar{H}^{\mathrm{p}}$ является Г-эквивариантно изометрически изоморфными $L^{2}(K, \mu) \otimes L$. Отсюда следует, что $\left.\bar{\pi}^{\mathrm{p}}\right|_{K}$ является кратным левого регулярного представления.

Используя вышеупомянутое отождествление, мы построим клеточно-матричное представление для операторов Гекке.

СлЕДСТвиЕ 24. Мы исполъзуем обозначения, введенные в лемме 23. Обозначим каноническую матричную единичу алгебры $B\left(l^{2}\left(\Gamma_{0} \backslash \Gamma\right)\right)$ через

$$
\left(e_{\Gamma_{0} s_{i}, \Gamma_{0} s_{j}}\right)_{i, j=1,2, \ldots,\left[\Gamma: \Gamma_{0}\right]} .
$$

Мъ используем изоморфизм

$$
B\left(H^{\Gamma_{0}}\right) \cong B\left(l^{2}\left(\Gamma_{0} \backslash \Gamma\right)\right) \otimes B(L),
$$

определенный выше. Тогда оператор Гекке $\left[\Gamma_{0}:\left(\Gamma_{0}\right)_{\sigma}\right] P_{H^{\Gamma_{0}}} \bar{\pi}^{\mathrm{p}}(\sigma) P_{H^{\Gamma_{0}}}$ nредставляется в виде

$$
\sum_{i, j} \sum_{\theta \in s_{i}^{-1} \Gamma_{0} \sigma \Gamma_{0} s_{j}} P_{L} \pi(\theta) P_{L} \otimes e_{\Gamma_{0} s_{i}, \Gamma_{0} s_{j}} .
$$

ДокАЗАТЕЛЬство. Это утверждение следует из того, что проекция на $L^{\Gamma_{0}}=$ $\bigoplus \pi\left(s_{i}\right) L$ - это $\sum \pi\left(s_{i}\right) P_{L} \pi\left(s_{i}\right)^{*}$, где $s_{i}$ - представители смежных классов, введенные формулировке теоремы 22. Мы используем представителей смежных классов, чтобы построить унитарный оператор, отображающий $L^{\Gamma_{0}}$ на $\ell^{2}\left(\Gamma_{0}\right) \otimes L$. Он будет отображать $\bigoplus \pi\left(s_{i}\right) l_{i}$ на $\bigoplus\left[\Gamma_{0} s_{i}\right] \otimes l_{i}$ для всех $l_{i} \in L$. Сопрягая оператор, заданный формулой (45), с помощью этого унитарного, получим (46).

Никакой дальнейшей перенормировки в формуле (46) не требуется, что можно непосредственно проверить, полагая $\sigma$ равным единичному элементу из $G$. Тогда для $\Gamma_{0} \in \mathscr{S}$ левая часть равенства (47) ниже есть $\operatorname{dim} H^{\Gamma_{0}}$. Так как $L$ является Г-блуждающим подпространством пространства $H$, правая часть в (47) подсчитывает, сколько раз единичный элемент попадает в $s_{i}^{-1} \Gamma_{0} s_{i}$, и умножает результат на размерность пространства $L$. Следствие доказано. 
ЗАмечАниЕ 25. В частности, если $L$ имеет конечную размерность, то справедлива следующая формула для следов операторов Гекке:

$$
\operatorname{Tr}\left(\left[\Gamma_{0}:\left(\Gamma_{0}\right)_{\sigma}\right]\left[P_{H^{\Gamma_{0}}} \bar{\pi}(\sigma) P_{H^{\Gamma_{0}}}\right]\right)=\sum_{i} \sum_{\theta \in s_{i}^{-1} \Gamma_{0} \sigma \Gamma_{0} s_{i}} \operatorname{Tr}\left(P_{L} \pi(\theta) P_{L}\right) .
$$

Заметим, что соответствие $\pi \rightarrow \bar{\pi}$ р также сохраняет функцию размерности $\operatorname{dim}_{\{\pi(\Gamma)\}^{\prime \prime}} H$. Это объясняется следующим результатом.

Лемма 26. Пусть $\pi$-унитарное представление как выше. Тогда справедливы следующие утверждения.

(i) Унитарное представление $\bar{\pi}^{\mathrm{p}}$ продолжается до представления $C^{*}$-алгебр полного свободного произведения с обгединением $C^{*}$-алгебр

$$
C^{*}(\bar{G}) *_{C *(K)} B\left(L^{2}(K)\right)
$$

в $B\left(\bar{H}^{\mathrm{p}}\right)$.

(ii) Унитарное представление $\bar{\pi}^{\mathrm{p}}$ группь $\bar{G}$ обладает тем свойством, что $\left.\bar{\pi}_{0}^{\mathrm{p}}\right|_{K}$ является конечным кратным левого регулярного представления группъи $K$ на $L^{2}(K, \mu)$.

ДокАзАТЕльство. Чтобы доказать п. (i), нужно построить представление алгебры $B\left(L^{2}(K)\right)$ в $B\left(\bar{H}^{\mathrm{p}}\right)$. Так как $B\left(L^{2}(K)\right)$ порождается алгеброй сверток с непрерывными функциями на $K$ и функциями из $C(K)$, рассматриваемыми как операторы умножения, достаточно описать представление алгебры $C(K)$ в $B\left(\bar{H}^{\mathrm{p}}\right)$, которое согласовано с представлением алгебры $C^{*}(K)$ в $B\left(\bar{H}^{\mathrm{p}}\right)$, полученным ограничением $\bar{\pi}^{\mathrm{p}}$ на $C^{*}(K)$. В силу инвариантности относительно сдвигов достаточно рассмотреть следующий случай. Пусть $K_{0}$ - замыкание в проконечной топологии подгруппы $\Gamma_{0} \in \mathscr{S}$. Тогда $\chi_{K_{0}}$, рассматриваемый как элемент алгебры $C(K)$, будет действовать на векторе вида $\sum_{\gamma_{0} \in \Gamma_{1}} \pi\left(\gamma_{0}\right) l, \Gamma_{1} \in \mathscr{S}$, $l \in L$, отображая его в $\sum_{\gamma_{0} \in \Gamma_{0} \cap \Gamma_{1}} \pi\left(\gamma_{0}\right) l$. Легко проверить, что это определяет представление $C^{*}$-алгебры $C^{*}(\bar{G}) *_{C^{*}(K)} B\left(L^{2}(K)\right)$.

Чтобы доказать п. (ii), заметим, что при ограничении $\bar{\pi}$ p на $K$ операторы $W^{\Gamma_{0}}, \Gamma_{0} \in \mathscr{S}$, становятся сплетающими операторами между $\overline{\left.\pi\right|_{K}}$ и представлением $\lambda_{K} \otimes \operatorname{Id}_{L}$, действующими на $L^{2}(K) \otimes L$. Лемма доказана.

\section{4. Примеры представлений и ассоциированных пространств Г-инвариантных векторов}

В этом разделе мы обсудим конкретные примеры, которые удовлетворяют аксиомам, введенным в определении 14, и к которым можно применить построение из предложения 20. В этом разделе мы будем свободно использовать обозначения из вышеупомянутого определения и последующие утверждения.

Следующий пример играет для унитарных представлений на пространствах Г-инвариантных векторов ту же роль, которую левое регулярное представление дискретной группы играет в пространстве представлений дискретной группы. Заманчиво назвать это представление регулярным. 
ПримеР 27. Пусть $\pi=\lambda_{G}$ - левое регулярное представление группы $G$, действующей на $H=l^{2}(G)$. В этом случае векторное пространство $\mathscr{V}$ есть линейное пространство функций на $G$. Для $\Gamma_{0} \in \mathscr{S}$ пространство $\mathscr{V} \Gamma_{0}$ есть пространство лево- $\Gamma_{0}$-инвариантных функций на $G$, т. е. функцией на $\Gamma_{0} \backslash G$. Тогда гильбертово пространство $H^{\Gamma_{0}}$ есть $l^{2}\left(\Gamma_{0} \backslash G\right)$. Скалярное произведение определено так, что если $\Gamma_{1} \subseteq \Gamma_{0}, \Gamma_{0}, \Gamma_{1} \in \mathscr{S}$, то включения

$$
l^{2}\left(\Gamma_{0} \backslash G\right) \subseteq l^{2}\left(\Gamma_{1} \backslash G\right)
$$

изометрические.

Ясно, что в этом случае $\mathscr{V}_{\infty}$ - гильбертово пространство пополнения пространства

$$
\bigvee_{\Gamma_{0} \in \mathscr{S}} l^{2}\left(\Gamma_{0} \backslash G\right)
$$

и совпадает с $L^{2}(\bar{G}, \mu)$. Тогда представление $\bar{\pi}^{\mathrm{p}}$ есть левое регулярное представление $\lambda_{\bar{G}}$, действующее на $L^{2}(\bar{G}, \mu)$.

Заметим, что здесь мы неявно использовали отождествление на векторных пространствах, имеющих в качестве базиса множество смежных классов. Это отождествление состоит в следующем:

$$
\left[\Gamma_{0} g\right]=\sum_{i}\left[\Gamma_{1} s_{i} g\right], \quad g \in G .
$$

Это равенство, после перенормировки скалярного произведения, влечет за собой существование изометрического вложения

$$
l^{2}\left(\Gamma_{0} \backslash G\right) \subseteq l^{2}\left(\Gamma_{1} \backslash G\right) .
$$

В частности, все квазирегулярные представления $\lambda_{G / \Gamma_{0}}$ группы $G$ на пространствах смежных классов являются подпредставлениями из $\left.\lambda_{\bar{G}}\right|_{G}$. Действительно, используя приведенное выше равенство, получаем, что для всех $\Gamma_{1} \in \mathscr{S}$

$$
l^{2}\left(G / \Gamma_{1}\right) \subseteq \bigvee_{\Gamma_{0} \in \mathscr{S}} l^{2}\left(\Gamma_{0} \backslash G\right) .
$$

Квазирегулярные представления входят с бесконечной кратностью в левое регулярное представление $\left.\lambda_{\bar{G}}\right|_{G}$, так как они коммутируют с правым действием группы $G$.

Стандартный выбор Г-блуждающего порождающего подпространства из $l^{2}(G)$ состоит в выборе представителей правых смежных классов $\mathscr{C} \subseteq G$ группы $\Gamma$ в $G$ (так что $G$ - объединение попарно непересекающихся подмножеств $\left.\bigcup_{\sigma \in \mathscr{C}} \Gamma \sigma\right)$. Так как $P_{H^{\Gamma_{0}}}$ - проекция на $l^{2}\left(\Gamma_{0} \backslash G\right)$, то используя вышеупомянутое построение, получаем стандартное представление операторов Гекке и алгебр Гекке [7], [4], [21], [51], [29].

Опишем второй стандартный пример конструкции из предложения 20, соответствующий случаю $\operatorname{dim}_{\{\pi(\Gamma)\}^{\prime \prime}} H=\infty$. 
ПримеР 28. Пусть $(\mathscr{X}, \nu)$ - бесконечномерное пространство, на котором $G$ действует сохраняющими меру преобразованиями. Предположим, что ограничение действия группы $G$ на $\Gamma$ допускает фундаментальную область $F$ в $\mathscr{X}$ с мерой $\nu(F)=1$. Для каждого $\Gamma_{0} \in \mathscr{S}$ зафиксируем систему представителей смежных классов:

$$
\Gamma=\bigcup \Gamma_{0} s_{i}
$$

Пусть

$$
F_{\Gamma_{0}}=\bigcup s_{i} F
$$

Тогда $F_{\Gamma_{0}}$ является фундаментальной областью для $\Gamma_{0}$. Перенормируем меру $\nu$ на $F_{\Gamma_{0}}$ :

$$
\nu_{\Gamma_{0}}=\frac{1}{\left[\Gamma: \Gamma_{0}\right]} \nu
$$

Выбор представителей индуцирует проекцию $\pi_{\Gamma_{0}}: F_{\Gamma_{0}} \rightarrow F$, которая отображает $s_{i} f$ в $f$ для $f \in F$. Рассматривая двойственные пространства, получаем изометрическое включение

$$
L^{2}(F, \nu) \subseteq L^{2}\left(F_{\Gamma_{0}}, \nu_{\Gamma_{0}}\right)
$$

Унитарное представление группы $G$ на $L^{2}(\mathscr{X}, \nu)$ является представлением Купмана

$$
\pi_{\text {Koop }}(g) f(x)=f\left(g^{-1} x\right), \quad x \in \mathscr{X}, \quad g \in G, \quad f \in L^{2}(\mathscr{X}, \nu) .
$$

Мы используем формализм из определения 14, и пусть $\mathscr{V}$ - линейное пространство измеримых функций на $\mathscr{X}$. Ясно, что подпространство $\mathscr{V} \Gamma_{0}$ составляют функции из $\mathscr{V}$, которые $\Gamma_{0}$-эквивариантны. Тогда $H^{\Gamma_{0}}$ канонически отождествляется с гильбертовым пространством $L^{2}\left(F_{\Gamma_{0}}, \nu_{\Gamma_{0}}\right)$. Это пространство также отождествляется с подпространством $\Gamma_{0}$-инвариантных функций на $\mathscr{X}$.

Ясно, что в этом случае гильбертово пространство $\bar{H}^{\mathrm{p}}$ изометрически изоморфно

$$
L^{2}(K, \mu) \otimes L^{2}(F, \nu)=L^{2}(K \times F, \mu \times \nu)
$$

и совпадает с $\left.\bar{\pi}^{\mathrm{p}}\right|_{K}$ - это просто $\operatorname{Id}_{L^{2}(F)} \otimes \lambda_{K}$, где $\lambda_{K}$ - левое регулярное представление группы $K$ на $L^{2}(K, \mu)$.

Вышеприведенное построение также доказывает, что представление $\left.\bar{\pi}_{\text {Коор }}^{\mathrm{p}}\right|_{G}$ само является унитарным представлением Купмана. Его несложно восстановить по исходному представлению $\pi$. Действительно, беря считающую меру $\varepsilon$ на Г, получаем изоморфизм пространств с мерой:

$$
(\mathscr{X}, \nu) \cong(\Gamma, \varepsilon) \times(F, \nu) .
$$

Действие группы $G$ на $\mathscr{X}$ при вышеупомянутом отождествлении описывается в терминах коцикла на $G \times F$ со значениями в $\Gamma$, где $\Gamma$ действует левым умножением на сомножитель $\Gamma$ в произведении $\Gamma \times F$. Заменяя сомножитель $\Gamma$ в этом произведении сомножителем $K$ в произведении $K \times F$, получаем сохраняющее меру действие группы $G$ на пространстве с мерой $(K \times F, \mu \times \nu)$, имеющее 
тот же коцикл, что и действие группы $G$ на $\Gamma \times F$. Тогда унитарное представление $\left.\bar{\pi}_{\text {Коор }}^{\mathrm{p}}\right|_{G}$ на самом деле является унитарным представлением Купмана, соответствующим действию группы $G$ на $(K \times F, \mu \times \nu)$.

В вышеупомянутом построении проекция $P_{L}$ является оператором умножения на характеристическую функцию $\chi_{F}$. Условие сходимости, требующее, чтобы сумма

$$
\sum_{\theta \in \Gamma \sigma \Gamma} P_{L} \pi_{\text {Koop }}(\theta) P_{L}
$$

сходилась, очевидно в этом случае, так как эта сумма есть оператор Гекке (см., например, [41]).

Ниже мы кратко опишем, как схему из предложения 20 можно использовать для пространств автоморфных форм. В следующем разделе это будет подробно изучено в более общей постановке.

ПримеР 29. Рассмотрим меры $d \nu_{n}(z)=(\operatorname{Im} z)^{n-2} d \bar{z} d z$ на $\mathbb{H}$. Пусть $\pi_{n}$, $n>1, n \in \mathbb{N},-$ дискретная серия [30] (проективных) унитарных представлений группы $\operatorname{PSL}(2, \mathbb{R})$. Эти представления действуют на гильбертовом пространстве $H_{n}=H^{2}\left(\mathbb{H}, \nu_{n}\right)$. Формула для них похожа на унитарное представление Купмана за исключением множителя модулярности (см., например, [30]). Рассмотрим также большее гильбертово пространство $\widehat{H}_{n}=L^{2}\left(\mathbb{H}, \nu_{n}\right), n \geqslant 1$.

Положим $G=\operatorname{PGL}(2, \mathbb{Z}[1 / p])$, где $p-$ простое число, и пусть $\Gamma=\operatorname{PSL}(2, \mathbb{Z})$. Если $\pi_{n}$ - проективное унитарное представление, т. е. если $n$ нечетно, то также задан 2-коцикл (выражающий проективность представления $\pi_{n}$ ). Этот коцикл в данном случае является $\mathbb{Z}_{2}$-значным и потому продолжается до 2-коцикла на пополнении Шлихтинга. В данном случае это пополнение есть PGL $\left(2, \mathscr{Q}_{p}\right)$, где $\mathscr{Q}_{p}$ - поле $p$-адических чисел.

В силу результатов работы [19; §3.3] отсюда следует, что $\left.\pi_{n}\right|_{\Gamma}$ является (не обязательно целым) кратным левого регулярного представления $\lambda_{\Gamma}$. Действительно,

$$
\operatorname{dim}_{\left\{\pi_{n}(\Gamma)\right\}^{\prime \prime}} H_{n}=\frac{n-1}{12} .
$$

Следовательно, если $(n-1) / 12$ не целое, то не существует гильбертова пространства $L$ такого, что $H_{n}$ и $l^{2}(\Gamma) \otimes L$ изоморфны как Г-модули. Более того, даже если $(n-1) / 12$ целое, не существует канонического выбора $L$, который позволил бы действовать так же, как в предложении 20.

Чтобы преодолеть эту трудность, мы используем Г-блуждающее порождающее подпространство представления $\widehat{\pi}_{n}$, которое содержит $\pi_{n}$ в качестве подпредставления. Положим $\widehat{\pi}_{n}$ равным унитарному представлению группы $\operatorname{PSL}(2, \mathbb{R})$, заданному той же алгебраической формулой на функциях на $\mathbb{H}$, что определяет представление $\pi_{n}$. То же самое вычисление, которое показывает, что $\pi_{n}$ - унитарное представление, так же просто доказывает, что $\widehat{\pi}_{n}-$ унитарное представление.

Хорошо известно (см., например, [23], [34]), что ассоциированное гильбертово пространство $H_{n}^{\Gamma}$ является конечномерным гильбертовым пространством, состоящим из автоморфных форм веса $n$ для группы $\Gamma=\operatorname{PSL}(2, \mathbb{Z})$. Чтобы применить формализм из определения 14 , положим $\mathscr{V}$ равным пространству 
аналитических функций на $\mathbb{H}$. В следующем разделе мы используем эту схему для вычисления следов операторов Гекке.

Затем для того, чтобы описать скалярное произведение на $H_{n}^{\Gamma}$, мы используем скалярное произведение гильбертова пространства из предыдущего примера для унитарного представления $\widehat{\pi}_{n}$. Если $F$ - фундаментальная область для действия группы $\Gamma$ на $\mathbb{H}$, то положим $P_{L}$ равным оператору умножения $M_{\chi_{F}}$ на характеристическую функцию $\chi_{F}$, действующему на $L^{2}\left(\mathbb{H}, \nu_{n}\right)$. Скалярное произведение в явном виде будет построено в следующем разделе (см. формулу (50)). В частном случае, рассматриваемом в настоящем примере, эта формула для скалярного произведения оказывается каноническим скалярным произведением Петерссона [34]. Описанная выше процедура будет использоваться ниже для получения явного описания операторов Гекке и для вычисления их следов.

Приведем еще один пример, где конструкция из предложения 20 применяется для построения Г-инвариантных векторов. Этот пример соответствует представлениям вида $\pi \otimes \pi^{\text {op }}$, где $\pi^{\text {op }}$ - комплексно-сопряженное.

ПримеР 30. Пусть $\Gamma \subseteq G, \pi, \pi_{0}, P_{0}, P_{L}$ такие же, как в определении 1 . Рассмотрим диагональное унитарное представление $\widetilde{\pi}=\pi_{0} \otimes \pi_{0}^{\text {op }}$ группы $G$. В этом примере для простоты изложения мы предполагаем, что все подгруппы из $\mathscr{S}$ имеют бесконечные нетривиальные классы сопряженности. Это соответствует тому, что все ассоциированные алгебры фон Неймана имеют единственные следы (являются факторами, т. е. не имеют центра).

Заметим, что даже если $\pi_{0}$ проективное, то представление $\pi_{0} \otimes \pi_{0}^{\text {op }}$ унитарное, без коцикла. Более того, так как в этом случае размерность Мюррея-фон Неймана бесконечна, унитарное представление $\pi_{0} \otimes \pi_{0}^{\text {op }}$ удовлетворяет условиям предложения 20. Так как мы зарезервировали обозначение $\bar{H}_{0}^{\mathrm{p}}$ для других целей, мы используем здесь обозначение $H_{0}^{\text {op }}$ для гильбертова пространства, сопряженного к $H_{0}$.

Тогда представление $\pi_{0} \otimes \pi_{0}^{\text {op }}$ унитарно эквивалентно представлению $\mathrm{Ad} \pi_{0}$, определенному на $G$, со значениями в унитарной группе гильбертова пространства

$$
H_{0} \otimes H_{0}^{\mathrm{op}} \cong \mathscr{C}_{2}\left(H_{0}\right) \subseteq B\left(H_{0}\right)
$$

Здесь $\mathscr{C}_{2}\left(H_{0}\right)$ - идеал, состоящий из операторов Гильберта-Шмидта, действующих на $H_{0}$ (см. [50]). Формула для представления $\mathrm{Ad} \pi_{0}$ такова:

$$
\operatorname{Ad} \pi_{0}(g)(X)=\pi_{0}(g) X \pi_{0}(g)^{-1}, \quad X \in \mathscr{C}_{2}\left(H_{0}\right), \quad g \in G .
$$

Оно очевидным образом продолжается до (неунитарного) представления группы $G$ во (внутренние) автоморфизмы алгебры $B\left(H_{0}\right)$.

В схеме определения 14 положим пространство $\mathscr{V}$ равным $B\left(H_{0}\right)$. Тогда

$$
\mathscr{V}^{\Gamma}=\left\{\pi_{0}(\Gamma)\right\}^{\prime}=\{X \mid[X, \pi(\gamma)]=0 \text { для всех } \gamma \in \Gamma\} \subseteq B\left(H_{0}\right) .
$$

Более общо, для $\Gamma_{0} \in \mathscr{S}$ имеем

$$
\mathscr{V}^{\Gamma_{0}}=\left\{\pi\left(\Gamma_{0}\right)\right\}^{\prime} \subseteq B\left(H_{0}\right) .
$$


Так как мы предположили, что все группы $\Gamma_{0}$ из $\mathscr{S}$ имеют бесконечные нетривиальные классы сопряженности, то алгебры $\left\{\pi_{0}\left(\Gamma_{0}\right)\right\}^{\prime}, \Gamma_{0} \in \mathscr{S}$, являются факторами типа $\mathrm{II}_{1}$ и, следовательно, каждая из них наделена единственным нормированным следом $\tau_{\Gamma_{0}}$.

Пусть $\mathscr{A}_{\infty}$ - фактор типа $\mathrm{II}_{1}$, полученный как индуктивный, сохраняющий следы, направленный предел факторов $\left\{\pi_{0}\left(\Gamma_{0}\right)\right\}^{\prime}, \Gamma_{0} \in \mathscr{S}$. Тогда $\mathscr{A}_{\infty}$ имеет единственный след $\tau$, определяемый требованием

$$
\left.\tau\right|_{\left\{\pi_{0}\left(\Gamma_{0}\right)\right\}^{\prime}}=\tau_{\Gamma_{0}}, \quad \Gamma_{0} \in \mathscr{S} .
$$

Если $\sigma \in G$ и $\Gamma_{0} \in \mathscr{S}$, то оператор $\operatorname{Ad} \pi_{0}(\sigma)$ отображает $\left\{\pi_{0}\left(\Gamma_{0} \cap \Gamma_{\sigma^{-1}}\right)\right\}^{\prime}$ в $\left\{\pi_{0}\left(\sigma \Gamma_{0} \sigma^{-1} \cap \Gamma_{\sigma}\right)\right\}^{\prime}$. Значит, $\mathrm{Ad} \pi_{0}(\sigma)$ отображает $\mathscr{A}_{\infty}$ на $\mathscr{A}_{\infty}$. Таким образом, $\operatorname{Ad} \pi_{0}(\sigma), \sigma \in G$, продолжается до элемента группы автоморфизмов Aut $\mathscr{A}_{\infty}$ фактора $\mathscr{A}_{\infty}$.

Чтобы получить гильбертово пространство $\Gamma_{0}$-инвариантных векторов, мы используем стандартные $L^{2}$-пространства, ассоциированные с соответствующими $\mathrm{II}_{1}$-факторами (обозначения см., например, в [50]). Таким образом,

$$
\left(H_{0} \otimes H_{0}^{\mathrm{op}}\right)^{\Gamma_{0}}=L^{2}\left(\left\{\pi\left(\Gamma_{0}\right)\right\}^{\prime}, \tau_{\Gamma_{0}}\right) \quad \text { и } \quad{\overline{\left(H_{0} \otimes H_{0}^{\mathrm{op}}\right.}}^{\mathrm{p}}=L^{2}\left(\mathscr{A}_{\infty}, \tau\right) .
$$

В частности, если $\operatorname{dim}_{\{\pi(\Gamma)\}^{\prime \prime}} H_{0}=1$, то

$$
\left(H_{0} \otimes H_{0}^{\mathrm{op}}\right)^{\Gamma} \cong L^{2}(\mathscr{L}(\Gamma), \tau) \cong \ell^{2}(\Gamma) .
$$

Следовательно, унитарное представление $\operatorname{Ad} \pi(\sigma), \sigma \in G$, индуцирует унитарное представление

$$
\overline{\mathrm{Ad} \pi}^{\mathrm{op}}=\overline{\pi_{0} \otimes \pi_{0}^{\mathrm{op}}},
$$

соответствующее $\pi_{0} \otimes \pi_{0}^{\text {op }}$, как определяется в предложении 20 .

Хотя это не используется в настоящей работе, заметим, что в силу теории индексов Джоунса [25], отождествляя проекции Джоунса для включения

$$
\left\{\pi_{0}\left(\Gamma_{0}\right)\right\}^{\prime \prime} \subseteq\left\{\pi_{0}(\Gamma)\right\}^{\prime \prime}
$$

с характеристической функцией замыкания подгруппы $\Gamma_{0}$ в $K$, получаем (см. [39]), что $\mathscr{A}_{\infty}$ изоморфен алгебре фон Неймана со скрещенным произведением $\mathscr{L}\left(\Gamma \rtimes L^{\infty}(K, \nu)\right)$, где $\Gamma$ действует левыми сдвигами на $K$. Представление $\left.\overline{\operatorname{Ad} \pi}^{\mathrm{p}}\right|_{\Gamma}$ действует тождественно на $\mathscr{L}(\Gamma) \subseteq \mathscr{A}_{\infty}$ и правыми сдвигами на $K$.

\section{5. Построение представления $\bar{\pi}_{0}^{\mathrm{p}}$ при отсутствии Г-блуждающего порождающего подпространства}

В этом разделе мы анализируем случай унитарного представления $\pi_{0}$ группы $G$ на гильбертовом пространстве $H_{0}$, для которого $\operatorname{dim}_{\left\{\pi_{0}(\Gamma)\right\}^{\prime \prime}} H_{0}$ не обязательно целое. Мы предполагаем, что эта размерность - конечное положительное число. Таким образом, Г-блуждающее порождающее подпространство $L \subseteq H_{0}$ из определения 13 может не существовать.

Мы предполагаем, что выполняются условия из определения 1. Напомним, что в определении 1 требуется, чтобы существовало унитарное представление $\pi$ группы $G$ на большем гильбертовом пространстве $H$, обладающее 
Г-блуждающим порождающим подпространством $L$. Исходное представление $\pi_{0}$ должно быть подпредставлением представления $\pi$. Напомним, что, как и в определении 1 , мы обозначаем через $P_{L}$ и $P_{0}$ ортогональные проекции из $H$ на $L$ и $H_{0}$ соответственно.

Пространства $\Gamma_{0}$-инвариантных векторов строятся (см. предложение 20) как пространства формальных сумм по группе $\Gamma_{0} \in \mathscr{S}$. Следовательно, $\Gamma_{0}$-инвариантные векторы отождествляются, как и в разделе 3 , с $\Gamma_{0}$-инвариантными неограниченными линейными формами на гильбертовом пространстве $H_{0}$.

Использование вспомогательного представления $\pi$, обладающего Г-блуждающим порождающим подпространством и содержащего исходное представление $\pi_{0}$ в качестве подпредставления, подсказывается случаем автоморфных форм (см. описание в примере 29). Формально автоморфные формы - это векторы, неподвижные относительно $\left.\pi_{n}\right|_{\Gamma}$, действующего на гильбертовом пространстве $H_{n}$. Невозможно найти настоящие векторы с этим свойством, так как нет суммируемости с квадратом. С другой стороны, алгебраическая формула, определяющая представление $\pi_{n}$, допускает Г-инвариантные аналитические функции. Они являются автоморфными формами [23]. Чтобы построить структуру гильбертова пространства на пространстве автоморфных форм заданного веса, используется скалярное произведение Петерссона. Чтобы определить скалярное произведение Петерссона [34], используется фундаментальная область $F$ для действия группы $\Gamma$ на $\mathbb{H}$. Пространство $L^{2}\left(F, \nu_{n}\right)$ является Г-блуждающим порождающим подпространством для большего унитарного представления, содержащего $\pi_{n}$ в качестве подпредставления и действующего на $L^{2}\left(\mathbb{H}, \nu_{n}\right)$.

В рамках этого раздела унитарное представление $\pi_{0}$ есть $\pi_{n}$, а большее представление, обладающее Г-блуждающим порождающим подпространством, действует на $L^{2}\left(\mathbb{H}, \nu_{n}\right)$. Проекция $P_{L}$ есть оператор на $L^{2}\left(\mathbb{H}, \nu_{n}\right)$, действующий умножением на характеристическую функцию $\chi_{F}$ фундаментальной области. Кроме того, проекция $P_{0}$ есть проекция (Бергмана) на пространство аналитических функций $H^{2}\left(\mathbb{H}, \nu_{n}\right)$.

Ниже мы описываем изменения процедуры из предложения 20 и определения 14, которые нужны для рассмотрения настоящей ситуации. Мы начинаем с построения пространства $\Gamma_{0}$-инвариантных векторов.

Лемма 31. Рассмотрим группь $\Gamma \subseteq G$ и представление $\pi_{0}$ группы $G$ со свойствами из определения 1. Мы используем обозначения, введенные в этом определении.

Для $\Gamma_{0} \in \mathscr{S}$ зафиксируем систему представителей $s_{i}$ правых смежных классов для $\Gamma_{0}$ в $\Gamma$, так что $\Gamma=\bigcup \Gamma_{0} s_{i}$. Пусть $L^{\Gamma_{0}}$ - гильбертово пространство, введенное в (43), с нормой, подчиненной условию перенормировки из формуль (44) в теореме 22. Пусть $P_{L^{\Gamma_{0}}}$ - ортогональная проекция из $H$ на $L^{\Gamma_{0}}$.

Тогда формула

$$
\mathscr{P}_{\Gamma_{0}, L}=\sum_{\gamma \in \Gamma_{0}} P_{L^{\Gamma_{0}}} \pi_{0}(\gamma) P_{L^{\Gamma_{0}}} \in B\left(L^{\Gamma_{0}}\right)
$$

определяет проекиию в $B\left(L^{\Gamma_{0}}\right)$. 
ДокАзАтельство. Тот факт, что $\mathscr{P}_{\Gamma_{0}, L}-$ проекция и, более общо, тот факт, что формула (55) в теореме 34 определяет представление алгебры Гекке двойных смежных классов для $\Gamma_{0} \in \mathscr{S}$, вытекает непосредственно из следующего тождества, которое справедливо для $\sigma_{1}, \sigma_{2} \in G$ и $\Gamma_{0} \in \mathscr{S}$ :

$$
\sum_{\gamma \in \Gamma_{0}} P_{L^{\Gamma_{0}}} \pi_{0}\left(\sigma_{1} \gamma\right) P_{L^{\Gamma_{0}}} \pi_{0}\left(\gamma^{-1} \sigma_{2}\right) P_{L^{\Gamma_{0}}}=P_{L^{\Gamma_{0}}} \pi_{0}\left(\sigma_{1} \sigma_{2}\right) P_{L^{\Gamma_{0}}} .
$$

Сходимость ряда в левой части этого равенства следует из технического условия (v), налагаемого в определении 1. Здесь мы берем поточечное операторное произведение двух операторных рядов, которые сходятся в $\mathscr{C}_{2}(L)$. Формула (49) сразу следует из того, что

$$
\sum_{\gamma_{0} \in \Gamma_{0}} \pi(\gamma) P_{L^{\Gamma_{0}}} \pi\left(\gamma^{-1}\right)
$$

- единичный оператор на $H$. Лемма доказана.

Ниже мы адаптируем предложение 20 к контексту представления $\pi_{0}$ со свойствами, введенными в определении 1. В следующей лемме мы строим гильбертово пространство “виртуальных" Го-инвариантных векторов, ассоциированное с $\pi_{0}$ (мы используем обозначения и определения из леммы 31 ).

Лемма 32. Пусть $H_{0}^{\Gamma_{0}}$ есть пространство формальных рядов следующего вида:

$$
H_{0}^{\Gamma_{0}}=\left\{\sum_{\gamma \in \Gamma_{0}} \pi_{0}(\gamma) l \mid l \in L^{\Gamma_{0}}\right\}
$$

Пусть $\mathscr{D}_{L, \pi}$ определено формулой (30) из леммы 18. К пространству $H_{0}^{\Gamma_{0}}$ nрименяется то же отождествление, что и в формуле (33) из определения 19. Отсюда следует, что

(i) имеет место равенство

$$
H_{0}^{\Gamma_{0}}=\left\{\sum_{\gamma \in \Gamma_{0}} \pi_{0}(\gamma) h \mid h \in \mathscr{D}_{L, \pi}\right\}
$$

(ii) представление $\pi_{0}$ продолюсается до канонического представления $\pi_{0}^{\mathrm{p}}$ группы $G$ в группу линейных изоморфизмов векторного пространства $\underset{\Gamma_{0} \in \mathscr{S}}{\bigvee} H^{\Gamma_{0}}$.

ДокАзАтельство. Утверждение (i) является непосредственным следствием отождествления в формуле (33). Представление $\pi_{0}^{\mathrm{p}}$ из утверждения (ii) строится с помощью той же процедуры, что и в доказательстве предложения 20 (см. формулу (38)).

В следующей теореме мы определяем согласованное скалярное произведение на пространствах $\Gamma_{0}$-инвариантных векторов, введенных выше, $\Gamma_{0} \in \mathscr{S}$. Мы также строим унитарное представление на индуктивном пределе гильбертовых пространств $\Gamma_{0}$-инвариантных векторов. 
Теорема 33. Во введенном выше контексте справедливы следующие утверждения.

(i) Пусть $\Gamma_{0} \in \mathscr{S}$ u $h_{1}, h_{2} \in \mathscr{D}_{L, \pi}$. По аналогии с формулой (36) определим

$$
\left\langle\sum_{\gamma_{0} \in \Gamma_{0}} \pi_{0}\left(\gamma_{0}\right) h_{1}, \sum_{\gamma_{0}^{\prime} \in \Gamma_{0}} \pi_{0}\left(\gamma_{0}^{\prime}\right) h_{2}\right\rangle_{\infty}=\left\langle P_{L^{\Gamma_{0}}}\left(\sum_{\gamma_{0} \in \Gamma_{0}} \pi_{0}\left(\gamma_{0}\right) h_{1}\right), \sum_{\gamma_{0}^{\prime} \in \Gamma_{0}} \pi_{0}\left(\gamma_{0}^{\prime}\right) h_{2}\right\rangle .
$$

Для подгрупп $\Gamma_{1} \subseteq \Gamma_{0}$ из $\mathscr{S}$ включения $H_{0}^{\Gamma_{0}} \subseteq H_{0}^{\Gamma_{1}}$ изометрические. Поэтому (50) продолжсается до скалярного произведения $\langle\cdot, \cdot\rangle_{\infty}$ на $\underset{\Gamma_{0} \in \mathscr{S}}{\bigvee} H^{\Gamma_{0}}$.

(ii) Скалярное произведение $\langle\cdot, \cdot\rangle_{\infty}$ на пространстве $\underset{\Gamma_{0} \in \mathscr{S}}{\bigvee} H^{\Gamma_{0}}$ удовлетворяет условиям определения 14.

(iii) Напомним, что пространство $L^{\Gamma_{0}}$, введенное в (43), есть $\Gamma_{0}-$ блуждающее порождающее подпространство для представления $\left.\pi\right|_{\Gamma_{0}}$. Пусть $\widetilde{L}^{\Gamma_{0}}$ другое $\Gamma_{0}$-блуждающее порождающее подпространство пространства $Н$ для представления $\left.\pi\right|_{\Gamma_{0}}$. Предположим, что существуют два ортогональных разбиения

$$
\widetilde{L}^{\Gamma_{0}}=\bigoplus_{\gamma \in \Gamma_{0}} \widetilde{L}_{\gamma} \quad u \quad L^{\Gamma_{0}}=\bigoplus_{\gamma \in \Gamma_{0}} L_{\gamma}
$$

такие, что $\widetilde{L}_{\gamma}=\pi(\gamma) L_{\gamma}$ для $\gamma \in \Gamma_{0}$. Тогда при замене в формуле (50) пространства $L^{\Gamma_{0}}$ на $\widetilde{L}^{\Gamma_{0}}$ значение скалярного произведения не изменяется.

(iv) Пусть $\bar{H}_{0}^{\mathrm{p}}$ - гилъбертово пространство пополнения индуктивного предела $\bigvee_{\Gamma_{0}} H^{\Gamma_{0}}$ пространств, построенных в $n$. (i). Для $g \in G$ определим унитарное преобразование $\bar{\pi}_{0}^{\mathrm{p}}(g)$ на $\bar{H}_{0}^{\mathrm{p}}$ формулой (38) из доказательства предложения 20. Тогда $\bar{\pi}_{0}^{\mathrm{p}}$ - унитарное представление группы $G$ в унитарную группу гильбертова пространства $\bar{H}_{0}^{\mathrm{p}}$.

Прежде чем доказывать теорему, сделаем несколько замечаний. Формулу (50) в определении скалярного произведения можно также продолжить формулой

$$
\left\langle\sum_{\gamma_{0} \in \Gamma_{0}} \pi\left(\gamma_{0}\right) P_{0} h, \sum_{\gamma_{0}^{\prime} \in \Gamma_{0}} \pi\left(\gamma_{0}^{\prime}\right) P_{0} l\right\rangle_{\infty}
$$

Это доказывает, что скалярное произведение, которое мы определяем на $H_{0}^{\Gamma_{0}}$, согласуется со скалярным произведением на пространстве $H^{\Gamma_{0}}$, введенным в предложении 20.

В случае автоморфных форм, когда $\pi_{0}$ - представление $\pi_{n}$ и $P_{0}$ - проекция Бергмана на ассоциированное пространство интегрируемых с квадратом аналитических функций, технические условия (v), (vi) в определении 1 следуют из того, что воспроизводящее ядро для пространства автоморфных форм является суммой по Г воспроизводящих ядер, ограниченных на фундаментальную область, для операторов $\chi_{F} \pi_{0}(\gamma) \chi_{F}, \gamma \in \Gamma$. То же справедливо для суммы по любому двойному смежному классу - в этом случае сумма ядер равна воспроизводящему ядру для оператора Гекке, ассоциированного с двойным смежным классом. Сходимость воспроизводящих ядер выполняется в норме Гильберта-Шмидта [53]. Заметим, что в этой же работе [53] доказана абсолютная сходимость для суммы следов. 
Подобие формуле скалярного произведения Петерссона следует из того, что в частном случае, соответствующем автоморфным формам, проекция $P_{L}$ заменяется оператором проекции $M_{\chi_{F}}$, полученным умножением на характеристическую функцию $\chi_{F}$ фундаментальной области $F$. Тот факт, что $P_{0} M_{\chi_{F}}-$ оператор со следом, проверен в [19; § 3.3]. Формула (50) напоминает скалярное произведение Петерссона. Действительно, чтобы определить скалярное произведение Петерссона двух автоморфных форм $f, g$, используют $L^{2}$-скалярное произведение $\chi_{F} f$ и $g$.

Заметим, что операторы Гекке можно было определить непосредственно с помощью формулы (55) из теоремы 34 ниже. Там (см. также [40]) мы дадим прямое доказательство того, что (55) является представлением алгебры Гекке двойных смежных классов подгруппы $\Gamma_{0}$ в $G$. С другой стороны, использование пространства $H_{0}^{\Gamma_{0}}$ в качестве пространства усредненных сумм по Г влечет, что пространства Г-инвариантных векторов, которые мы рассматриваем в теореме 33, соответствуют пространствам автоморфных форм.

Преимущество подхода, рассматриваемого в теореме 33 , состоит в том, что мы имеем конкретные формулы для унитарного представления $\bar{\pi}_{0}^{\mathrm{p}}$, описанные непосредственно в терминах исходных представлений $\pi_{n}$ и их взаимодействия с $P_{0} M_{\chi_{F}}$. Это будет использовано в настоящей работе для вычислений следов и характеров ассоциированных унитарных представлений.

ДоКАЗАТЕЛЬСТво теОРЕмы 33. Мы используем тот факт, что сумма в формуле (48) из леммы 31 определяет конечномерную проекцию. Тогда скалярное произведение (50) равно

$$
\begin{gathered}
\left\langle P_{L^{\Gamma_{0}}}\left(\sum_{\gamma_{0} \in \Gamma_{0}} \pi_{0}\left(\gamma_{0}\right) h_{1}\right), P_{L^{\Gamma_{0}}}\left(\sum_{\gamma_{0}^{\prime} \in \Gamma_{0}} \pi_{0}\left(\gamma_{0}^{\prime}\right) h_{2}\right)\right\rangle \\
=\left\langle\mathscr{P}_{\Gamma_{0}, L} h_{1}, \mathscr{P}_{\Gamma_{0}, L} h_{2}\right\rangle=\left\langle\mathscr{P}_{\Gamma_{0}, L} h_{1}, h_{2}\right\rangle,
\end{gathered}
$$

что - ввиду того, что $\mathscr{P}_{\Gamma_{0}, L}-$ конечномерная проекция в $L^{\Gamma_{0}},-$ является корректно определенным скалярным произведением на пространстве $H_{0}^{\Gamma_{0}}$.

Тот факт, что для $\Gamma_{0}, \Gamma_{1} \in \mathscr{S}$ таких, что $\Gamma_{1} \subseteq \Gamma_{0}$, включения $H_{0}^{\Gamma_{0}} \subseteq H_{0}^{\Gamma_{1}}$ изометрические, доказывается в точности так же, как в предложении 20. Утверждение (iі) является несложным следствием определения (50) скалярного произведения на $\Gamma_{0}$-инвариантных векторах. Оно соответствует независимости от выбора фундаментальной области в формуле скалярного произведения Петерссона.

Докажем, что выполнено условие инвариантности (ii.3) из определения 14. Мы покажем, что $\pi_{0}^{\mathrm{p}}(\sigma), \sigma \in G$, изометрически отображает $H_{0}^{\Gamma_{\sigma}-1}$ на $H_{0}^{\Gamma_{\sigma}}$. Как и выше, зафиксируем $h_{1}, h_{2}$ в $\mathscr{D}_{L, \pi}$. Тогда, используя формулу $(51)$ и конструкцию представления $\pi_{0}^{\mathrm{p}}$, получаем

$$
\begin{aligned}
\left\langle\pi_{0}^{\mathrm{p}}(\sigma)\right. & \left.\left(\sum_{\gamma_{0} \in \Gamma_{\sigma}-1} \pi_{0}\left(\gamma_{0}\right) h_{1}\right), \pi_{0}^{\mathrm{p}}(\sigma)\left(\sum_{\gamma_{0}^{\prime} \in \Gamma_{\sigma^{-1}}} \pi_{0}\left(\gamma_{0}^{\prime}\right) h_{2}\right)\right\rangle_{\infty} \\
= & \left\langle\sum_{\gamma_{0} \in \Gamma_{\sigma}} \pi_{0}\left(\gamma_{0}\right)\left(\pi_{0}(\sigma) h_{1}\right), \sum_{\gamma_{0}^{\prime} \in \Gamma_{\sigma}} \pi_{0}\left(\gamma_{0}^{\prime}\right)\left(\pi_{0}(\sigma) h_{2}\right)\right\rangle_{\infty} \\
= & \left\langle P_{L^{\Gamma} \sigma}\left[\sum_{\gamma_{0} \in \Gamma_{\sigma}} \pi_{0}\left(\gamma_{0}\right)\left(\pi_{0}(\sigma) h_{1}\right)\right],\left(\pi_{0}(\sigma) h_{2}\right)\right\rangle,
\end{aligned}
$$


что, в силу унитарности $\pi_{0}(\sigma)$ на $H_{0}$, равно

$$
\left\langle\left[\pi\left(\sigma^{-1}\right) P_{L^{\Gamma} \sigma} \pi(\sigma)\right] \pi_{0}\left(\sigma^{-1}\right)\left[\sum_{\gamma_{0} \in \Gamma_{\sigma}} \pi_{0}\left(\gamma_{0}\right)\left(\pi_{0}(\sigma) h_{1}\right)\right], h_{2}\right\rangle .
$$

Здесь $\pi\left(\sigma^{-1}\right) P_{L^{\Gamma} \sigma} \pi(\sigma)$ - проекция на пространство $\widetilde{L}=\pi\left(\sigma^{-1}\right) L^{\Gamma_{\sigma}}$. Это пространство является $\Gamma_{\sigma^{-1}}$-блуждающим порождающим подпространством для представления $\left.\pi\right|_{\Gamma_{\sigma-1}}$. Обозначим через $P_{\widetilde{L}}$ ортогональную проекцию на $\widetilde{L}$. Тогда (52) равно

$$
\left\langle P_{\widetilde{L}}\left(\sum_{\gamma_{0} \in \Gamma_{\sigma^{-1}}} \pi_{0}\left(\gamma_{0}\right) h_{1}\right), h_{2}\right\rangle
$$

В силу технического условия (ii) определения 1 подпространство $\widetilde{L}$ эквивалентно подпространству $L^{\Gamma_{\sigma}-1}$ в смысле п. (iii) настоящей теоремы. Поэтому, ввиду п. (iii), получаем, что (53) равно

$$
\left\langle P_{L^{\Gamma} \sigma^{-1}}\left(\sum_{\gamma_{0} \in \Gamma_{\sigma^{-1}}} \pi_{0}\left(\gamma_{0}\right) h_{1}\right), h_{2}\right\rangle=\left\langle\sum_{\gamma_{0} \in \Gamma_{\sigma^{-1}}} \pi_{0}\left(\gamma_{0}\right) h_{1}, \sum_{\gamma_{0}^{\prime} \in \Gamma_{\sigma^{-1}}} \pi_{0}\left(\gamma_{0}^{\prime}\right) h_{2}\right\rangle_{\infty} .
$$

В силу определения скалярного произведения в п. (i), эта цепочка равенств доказывает, что $\pi_{0}^{\mathrm{p}}(\sigma)$ изометрически отображает $H_{0}^{\Gamma_{\sigma-1}}$ на $H_{0}^{\Gamma_{\sigma}}$. Более общо, для $\Gamma_{0} \in \mathscr{S}$ рассуждение того же типа дает, что $\pi_{0}^{\mathrm{p}}(\sigma)$ отображает $H_{0}^{\Gamma_{\sigma}{ }^{-1} \cap \Gamma_{0}}$ на $H_{0}^{\Gamma_{\sigma} \cap \sigma \Gamma_{0} \sigma^{-1}}$. Поэтому условие инвариантности (ii.3) из определения 14 в данном случае выполняется. Пункт (iv) является несложным следствием предыдущего рассуждения. Теорема доказана.

В следующей теореме мы описываем унитарно эквивалентное представление операторов Гекке, действующих на пространствах $\Gamma_{0}$-инвариантных векторов, введенных в теореме 33. Ниже мы докажем, что проекция $\mathscr{P}_{\Gamma_{0}, L} \in$ $B\left(L^{\Gamma_{0}}\right)$, определенная формулой (48), унитарно эквивалентна другой проекции, определенной формулой (56). Это полезно для вычисления размерностей $\Gamma_{0}$-инвариантных векторов. Дело в том, что мы доказываем, что образ $\mathscr{P}_{\Gamma_{0}, L}$, который является подпространством из $L^{\Gamma_{0}}$, унитарно эквивалентен пространству векторов, ассоциированных с $H_{0}$, которые неподвижны относительно $\Gamma_{0}$.

Формула воспроизводящего ядра для проекции на пространство автоморфных форм и формула воспроизводящего ядра для операторов Гекке, полученные в [53], доказывают, что пространства $H_{0}^{\Gamma_{0}}$ и соответствующее действие операторов Гекке на пространствах $\Gamma_{0}$-инвариантных векторов, введенные в лемме 32 и в следующей теореме, такие же (для верхней полуплоскости), как в классическом случае.

ТеОрема 34. Предположим, что выполнены условия теоремы 33. Пусть $\Gamma_{0} \in \mathscr{S}$. По лемме 21 представление алгебры Гекке $\mathscr{H}_{0}\left(\Gamma_{0}, G\right)$, ассоииированное с представлением $\pi_{0}$, определяется соответствием

$$
\left[\Gamma_{0} \sigma \Gamma_{0}\right] \rightarrow\left[\Gamma_{0}:\left(\Gamma_{0}\right)_{\sigma}\right] P_{H_{0}^{\Gamma_{0}}} \bar{\pi}_{0}^{\mathrm{p}}(\sigma) P_{H_{0}^{\Gamma_{0}}}, \quad \sigma \in G .
$$

Тогда справедливы следующие утверждения. 
(i) Рассмотрим бесконечные суммы по смежным классам подгруппы $\Gamma_{0}$, которые сходятся в силу условий (v), (vi) определения 1:

$$
A_{0}\left(\Gamma_{0} \sigma \Gamma_{0}\right)=\sum_{\theta \in \Gamma_{0} \sigma \Gamma_{0}} P_{L^{\Gamma_{0}}} \pi_{0}(\theta) P_{L^{\Gamma_{0}}}, \quad \sigma \in G
$$

тогда

$$
A_{0}\left(\Gamma_{0} \sigma \Gamma_{0}\right)=\mathscr{P}_{\Gamma_{0}, L} A_{0}\left(\Gamma_{0} \sigma \Gamma_{0}\right) \mathscr{P}_{\Gamma_{0}, L},
$$

u поэтому $A_{0}\left(\Gamma_{0} \sigma \Gamma_{0}\right)$ лежит в $B\left(\mathscr{P}_{\Gamma_{0}, L} L^{\Gamma_{0}}\right)$.

(ii) Соответствие

$$
\left[\Gamma_{0} \sigma \Gamma_{0}\right] \rightarrow A_{0}\left(\Gamma_{0} \sigma \Gamma_{0}\right), \quad \sigma \in G
$$

определяет *-представление алгебры $\mathscr{H}_{0}\left(\Gamma_{0}, G\right)$ в $B\left(P_{\Gamma_{0}, L} L^{\Gamma_{0}}\right)$. Это представление унитарно эквивалентно *-представлению (см. (54)) алгебры Гекке с помощъю операторов Гекке, ассочиированных с представлением $\pi_{0}$.

Заметим, что оператор $A\left(\Gamma_{0}\right)=\mathscr{P}_{\Gamma_{0}, L}$ унитарно эквивалентен проекции на $\Gamma_{0}$-инвариантные векторы.

ДокАзАТЕльство сходно с доказательством теоремы 22. Так же, как в теореме 22 и ее доказательстве, задавая скалярное произведение формулой (50), для $\Gamma_{0} \in \mathscr{S}$ можно определить частичные изометрии $W^{\Gamma_{0}}: L^{\Gamma_{0}} \rightarrow H^{\Gamma_{0}}$ формулой

$$
W^{\Gamma_{0}} l=\sum_{\gamma \in \Gamma_{0}} \pi_{0}(\gamma) l, \quad l \in L^{\Gamma_{0}} .
$$

В отличие от случая, рассмотренного в теореме 22 , операторы $W^{\Gamma_{0}}$ являются частичными изометриями, имеющими в качестве исходного пространства проекцию $\mathscr{P}_{\Gamma_{0}, L}$, введенную в (48), с образами, равными пространствам $H^{\Gamma_{0}}$, $\Gamma_{0} \in \mathscr{S}$.

Мы используем частичные изометрии $W^{\Gamma_{0}}$ с исходным пространством, являющимся проекцией $\mathscr{P}_{\Gamma_{0}, L}$ в $B\left(L^{\Gamma_{0}}\right)$. Тогда $W^{\Gamma_{0}}$ унитарно преобразует оператор Гекке $P_{H^{\Gamma_{0}}} \bar{\pi}^{\mathrm{p}}(\sigma) P_{H^{\Gamma_{0}}}, \sigma \in G$, в выражение в формуле (55).

Формула (49) показывает, что для всех $\Gamma_{0} \in \mathscr{S}$ и $\sigma \in G$

$$
A_{0}\left(\Gamma_{0} \sigma \Gamma_{0}\right)=A_{0}\left(\Gamma_{0} \sigma \Gamma_{0}\right) \mathscr{P}_{\Gamma_{0}, L}=\mathscr{P}_{\Gamma_{0}, L} A_{0}\left(\Gamma_{0} \sigma \Gamma_{0}\right) .
$$

В силу той же формулы операторы

$$
A_{0}\left(\Gamma_{0} \sigma \Gamma_{0}\right), \quad \sigma \in G,
$$

определяют представление алгебры Гекке двойных смежных классов подгруппы $\Gamma_{0}$ в $G$. Теорема доказана.

Теорема 34 дает явное представление операторов Гекке, ассоциированных с представлением $\bar{\pi}_{0}^{\mathrm{p}}$ группы $\bar{G}$ в $\bar{H}_{0}^{\mathrm{p}}$, напрямую используя информацию из исходного представления $\bar{\pi}_{0}^{\mathrm{p}}$. Мы подытоживаем это в теореме 2 . 
ДокАЗАТЕЛЬСтво теоремы 2 . Пусть $L^{\Gamma_{0}}$ - подпространство, заданное формулой (43) и наделенное нормированным скалярным произведением, заданным формулой (44). С использованием теоремы 34 доказательство формулы (4) становится идентичным доказательству соответствующей формулы (46) в следствии 24. Мы выбираем представителей смежных классов так, как указано в формулировке теоремы. Для перехода от формулы (55) к формуле (4) надо просто использовать унитарный оператор

$$
\ell^{2}\left(\Gamma_{0} \backslash \Gamma\right) \otimes L \cong \bigoplus\left[\Gamma_{0} s_{i}\right] \otimes L, \quad L^{\Gamma_{0}}=\bigoplus \pi\left(s_{i}\right) L,
$$

отображающий $\bigoplus\left[\Gamma_{0} s_{i}\right] \otimes l_{i}$ на $\bigoplus \pi\left(s_{i}\right) l_{i}$ при $l_{i} \in L$.

Когда $\sigma$ является единицей в формуле (4), получаем проекцию

$$
\widetilde{\mathscr{P}}_{\Gamma_{0}, L}=\sum_{i, j} \sum_{\gamma \in s_{i}^{-1} \Gamma_{0} s_{j}} P_{L} \pi_{0}(\gamma) P_{L} \otimes e_{\Gamma_{0} s_{i}, \Gamma_{0} s_{j}}
$$

Следовательно, операторы в формуле (4) принадлежат алгебре

$$
\widetilde{\mathscr{P}}_{\Gamma_{0}, L}\left[B\left(l^{2}\left(\Gamma_{0} \backslash \Gamma\right)\right) \otimes B(L)\right] \widetilde{\mathscr{P}}_{\Gamma_{0}, L}
$$

ЗАмЕчАНИЕ 35. Пусть выполнены условия теоремы 34. В силу формулы (4) один из эквивалентных методов построения представления операторов Гекке состоит в следующем. Как и в примере 27, рассмотрим векторное пространство

$$
\mathscr{V}^{\Gamma_{0}}=l^{2}\left(\Gamma_{0} \backslash G\right), \quad \Gamma_{0} \in \mathscr{S} .
$$

На $\mathscr{V}^{\Gamma_{0}}$ определим скалярное произведение как линейное продолжение следующей билинейной формы:

$$
\left\langle\Gamma_{0} \sigma_{1}, \Gamma_{0} \sigma_{2}\right\rangle_{\pi_{0}}=\frac{1}{\left[\Gamma: \Gamma_{0}\right]} \sum_{\theta \in \sigma_{1}^{-1} \Gamma_{0} \sigma_{2}} \operatorname{Tr}\left(P_{L} \pi_{0}(\theta) P_{L}\right), \quad \Gamma_{0} \in \mathscr{S}, \quad \sigma_{1}, \sigma_{2} \in G .
$$

Для $\Gamma_{0} \in \mathscr{S}$ рассмотрим обычное алгебраическое представление операторов Гекке на $\mathbb{C}\left(\Gamma_{0} \backslash G\right)$. Рассматривая на $\mathbb{C}\left(\Gamma_{0} \backslash G\right)$ введенное выше скалярное произведение $\langle\cdot, \cdot\rangle_{\pi_{0}}$, получаем представление алгебры Гекке, унитарно эквивалентное представлению, заданному формулой (4).

Это соответствует рассмотрению состояния $\varepsilon$ на $C^{*}(\bar{G})$, определенного формулой

$$
\varepsilon\left(\chi_{\sigma_{1}^{-1} \Gamma_{0} \sigma_{2}}\right)=\frac{1}{\left[\Gamma: \Gamma_{0}\right]} \sum_{\theta \in \sigma_{1}^{-1} \Gamma_{0} \sigma_{2}} \operatorname{Tr}\left(P_{L} \pi_{0}(\theta) P_{L}\right), \quad \Gamma_{0} \in \mathscr{S}, \quad \sigma_{1}, \sigma_{2} \in G .
$$

Состояние $\varepsilon$ не может быть одновременно использовано на всех уровнях $\Gamma_{0} \in \mathscr{S}$ из-за перенормировочного множителя $1 /\left[\Gamma: \Gamma_{0}\right]$. Заметим, что состояние $\varepsilon$ фактически является композицией следа с семейством вполне положительных отображений, построенных в теореме 37. 


\section{6. Значения характера $\theta_{\pi_{0}}$, ассоциированного с представлением $\pi_{0}$}

В этом разделе мы выводим формулу следа для представления $\bar{\pi}_{0}^{\mathrm{p}}$. Заметим, что $\bar{\pi}_{0}^{\mathrm{p}}$ есть представление типа I $C^{*}$-алгебры $C^{*}(\bar{G})$. Согласно предложению 17 , представление $\bar{\pi}_{0}^{\mathrm{p}}$ продолжается до представления алгебры $\mathscr{A}(G, \bar{G})$ (или $\mathscr{A}_{\epsilon}(G, \bar{G})$, если имеется 2-коцикл). По теореме 2 имеем формулу для оператора Гекке $P_{H_{0}^{\Gamma_{0}}} \bar{\pi}_{0}^{\mathrm{p}}(\sigma) P_{H_{0}^{\Gamma_{0}}}$, ассоциированного с представлением $\bar{\pi}_{0}^{\mathrm{p}}$. Как объясняется ниже, это связывает формулу следа для представления $\bar{\pi}_{0}^{\mathrm{p}}$ с формулой следа для представления $\pi_{0}$.

Следующая лемма доказана в [12] (см. [12; формула (13)]). Здесь мы приводим другое доказательство.

Лемма 36. Характер $\theta_{\bar{\pi}_{0}^{\mathrm{p}}}=$ "Тr $\bar{\pi}_{0}^{\mathrm{p}}$ " представления $\bar{\pi}_{0}^{\mathrm{p}}$ въичисляется по формуле (7).

ДокАЗАТЕЛЬСтво. Используя тот факт, что характер локально интегрируем [45], получаем следующую формулу:

$$
\theta_{\bar{\pi}_{0}^{\mathrm{p}}}(\sigma)=\lim _{\Gamma_{0} \downarrow e, \Gamma_{0} \in \mathscr{S}} \frac{1}{\mu\left(\bar{\Gamma}_{0} \sigma \bar{\Gamma}_{0}\right)} \operatorname{Tr}\left(\bar{\pi}_{0}^{\mathrm{p}}\left(\chi_{\bar{\Gamma}_{0} \sigma \bar{\Gamma}_{0}}\right)\right), \quad \sigma \in G .
$$

В обозначениях, введенных перед леммой 21, имеем

$$
\mu\left(\bar{\Gamma}_{0} \sigma \bar{\Gamma}_{0}\right)=\left[\Gamma_{0}:\left(\Gamma_{0}\right)_{\sigma}\right] \mu\left(\bar{\Gamma}_{0} \sigma\right), \quad \Gamma_{0} \in \mathscr{S}, \quad \sigma \in G .
$$

Мера $\mu$ получена из меры Хаара на проконечном пополнении группы $\Gamma$ и, в силу общих предположений, бивариантна на $\bar{G}$; поэтому правая часть последнего равенства есть

$$
\left[\Gamma_{0}:\left(\Gamma_{0}\right)_{\sigma}\right] \frac{1}{\left[\Gamma: \Gamma_{0}\right]} .
$$

Следовательно, правая часть в (57) равна

$$
\lim _{\Gamma_{0} \downarrow e, \Gamma_{0} \in \mathscr{S}} \frac{\left[\Gamma: \Gamma_{0}\right]}{\left[\Gamma_{0}:\left(\Gamma_{0}\right)_{\sigma}\right]} \operatorname{Tr}\left(\bar{\pi}_{0}^{\mathrm{p}}\left(\chi_{\bar{\Gamma}_{0} \sigma \bar{\Gamma}_{0}}\right)\right) .
$$

Принимая во внимание формулу (39) леммы 21, получаем, что последнее выражение равно

$$
\begin{aligned}
& \lim _{\Gamma_{0} \downarrow e, \Gamma_{0} \in \mathscr{S}}\left[\Gamma: \Gamma_{0}\right]^{2} \operatorname{Tr}\left(\bar{\pi}_{0}^{p}\left(\chi_{\bar{\Gamma}_{0}}\right) \bar{\pi}_{0}^{\mathrm{p}}(\sigma) \bar{\pi}_{0}^{\mathrm{p}}\left(\chi_{\bar{\Gamma}_{0}}\right)\right) \\
& \quad=\lim _{\Gamma_{0} \downarrow e, \Gamma_{0} \in \mathscr{S}} \frac{1}{\left(\mu\left(\bar{\Gamma}_{0}\right)\right)^{2}} \operatorname{Tr}\left(\bar{\pi}_{0}^{\mathrm{p}}\left(\chi_{\bar{\Gamma}_{0}}\right) \bar{\pi}_{0}^{\mathrm{p}}(\sigma) \bar{\pi}_{0}^{\mathrm{p}}\left(\chi_{\bar{\Gamma}_{0}}\right)\right) .
\end{aligned}
$$

Если $K_{0}$ - замыкание подгруппы из $\mathscr{S}$, то в $C^{*}(\bar{G})$, используя произведение сверточных операторов, имеем $\left(\chi_{K_{0}}\right)^{2}=\mu\left(K_{0}\right) \chi_{K_{0}}$. Поэтому $\frac{1}{\mu\left(K_{0}\right)} \chi_{K_{0}}$ является проекцией. Обозначим через $\widetilde{\chi}_{K_{0}}$ перенормированный сверточный оператор $\frac{1}{\mu\left(K_{0}\right)} \chi_{K_{0}}$. Тогда правая часть в (58) принимает вид

$$
\lim _{\Gamma_{0} \downarrow e, \Gamma_{0} \in \mathscr{S}} \operatorname{Tr}\left(\bar{\pi}_{0}^{\mathrm{p}}\left(\widetilde{\chi}_{\bar{\Gamma}_{0}}\right) \bar{\pi}_{0}^{\mathrm{p}}(\sigma) \bar{\pi}_{0}^{\mathrm{p}}\left(\widetilde{\chi}_{\bar{\Gamma}_{0}}\right)\right)
$$


Так как $\bar{\pi}_{0}^{\mathrm{p}}-$ представление алгебры $C^{*}(G, \bar{G})$, это равно

$$
\lim _{\Gamma_{0} \downarrow e, \Gamma_{0} \in \mathscr{S}} \operatorname{Tr}\left(P_{H_{0}^{\Gamma_{0}}} \bar{\pi}_{0}^{\mathrm{p}}(\sigma) P_{H_{0}^{\Gamma_{0}}}\right) .
$$

Лемма доказана.

ДокАЗАТЕЛЬСтво СЛЕДствия 4. Формула (8) вытекает непосредственно из (7) и (5). Сгруппируем слагаемые суммы из формулы (8) в соответствии с классами сопряженности и возьмем предел. Тот факт, что группа $\Gamma_{g}^{\mathrm{st}}$ тривиальна, запрещает любому $\gamma \in \Gamma$ появляться более одного раза в сумме из формулы (8). Абсолютная сходимость сумм, участвующих в предельном переходе (определение 1), влечет второе утверждение следствия.

ДоКАЗАТЕЛЬСТВо СЛЕДСТВИЯ 7. Напомним, что для группового элемента $g \in G$ стабилизатор $\Gamma_{g}^{\text {st }}$ - это подгруппа $\{\gamma \mid \gamma g=g \gamma\}$. Пусть $\sigma$ - элемент из $G=\operatorname{PGL}(2, \mathbb{Z}[1 / p])$ такой, что $\Gamma_{\sigma}^{\text {st }}$ тривиален. Тогда в формуле (9) сумму

$$
\sum_{\gamma \in \Gamma} \operatorname{Tr}\left(P_{L} \pi_{n}\left(\gamma \sigma \gamma^{-1}\right)\right)
$$

можно вычислить напрямую, используя функцию символа Березина [3].

Действительно, эту сумму можно преобразовать следующим образом:

$$
\begin{gathered}
\sum_{\gamma \in \Gamma} \operatorname{Tr}_{B\left(H_{n}\right)}\left(M_{\chi_{F}} \pi_{0}(\gamma) \pi_{0}(\sigma) \pi_{0}\left(\gamma^{-1}\right)\right)=\sum_{\gamma \in \Gamma} \operatorname{Tr}_{B\left(H_{n}\right)}\left(M_{\chi_{F}} \pi(\gamma) \pi_{0}(\sigma) \pi\left(\gamma^{-1}\right)\right) \\
=\sum_{\gamma \in \Gamma} \operatorname{Tr}_{B\left(H_{n}\right)}\left(\pi\left(\gamma^{-1}\right) M_{\chi_{F}} \pi(\gamma) \pi_{0}(\sigma)\right)=\sum_{\gamma \in \Gamma} \operatorname{Tr}_{B\left(H_{n}\right)}\left(M_{\gamma \chi_{F}} \pi_{0}(\sigma)\right) .
\end{gathered}
$$

Пусть $d \nu_{0}(z)=(\operatorname{Im} z)^{-2} d \bar{z} d z-$ каноническая $\operatorname{PSL}(2, \mathbb{R})$-инвариантная мера на $\mathbb{H}$ и $\widehat{\pi_{n}(\theta)}(\bar{z}, z), z \in \mathbb{H},-$ контравариантный символ Березина [3] унитарного оператора $\widehat{\pi_{n}(\theta)}$. Тогда правая часть приведенной выше цепочки равенств есть не что иное, как

$$
\sum_{\gamma \in \Gamma} \int_{\gamma F} \widehat{\pi_{n}(\theta)}(\bar{z}, z) d \nu_{0}(z)
$$

что, в свою очередь, равно

$$
\int_{\mathbb{H}} \widehat{\pi_{n}(\theta)}(\bar{z}, z) d \nu_{0}(z) .
$$

Это последнее выражение - характер “ $\operatorname{Tr} \pi_{n}(\sigma)$ " представления $\pi_{n}$ (см. [32]). (Формула для суммы (59) другим способом получена в [53].) Следствие 7 доказано. 
ДокАЗАТЕЛЬСтво ЛЕммы 5. Обозначим гильбертово пространство, на котором действует представление $\bar{\pi}_{0}^{R}$, через $H_{0}^{R}$. Тогда

$$
\begin{aligned}
& \operatorname{Tr}_{B\left(H_{0}^{R}\right)}\left(\int_{\bar{G}^{R}} f(g) \bar{\pi}_{0}^{R}(g) d g\right)=\sum_{\gamma} \operatorname{Tr}_{B\left(H_{0}^{R}\right)}\left(P_{[\pi(\gamma) L]} \int_{\bar{G}^{R}} f(g) \bar{\pi}_{0}^{R}(g) d g\right) \\
& =\sum_{\gamma} \int_{\bar{G}^{R}} f(g) \operatorname{Tr}_{B\left(H_{0}^{R}\right)}\left(P_{\pi(\gamma) L} \bar{\pi}_{0}^{R}(g)\right) d g \\
& =\sum_{\gamma \in \Gamma} \int_{\bar{G}^{R}} f(g) \operatorname{Tr}_{B(L)}\left(P_{L} \bar{\pi}_{0}^{R}\left(\gamma g \gamma^{-1}\right) P_{L}\right) d g \\
& =\int_{\bar{G}^{R}} f(g) \sum_{\gamma} \operatorname{Tr}_{B(L)}\left(P_{L} \bar{\pi}_{0}^{R}\left(\gamma g \gamma^{-1}\right) P_{L}\right) d g .
\end{aligned}
$$

Утверждение (ii) вытекает из следствия 4. Лемма доказана.

\section{7. Случай, когда представление $\pi$ допускает "квадратный корень" $\pi_{0} \otimes \pi_{0}^{\mathrm{op}}$}

В этом разделе изучается случай, когда унитарное представление $\pi$ из раздела 3 допускает квадратный корень $\pi_{0} \otimes \pi_{0}^{\mathrm{op}}$, где $\pi_{0}-$ (проективное) унитарное представление, рассмотренное в разделе 5 . Так как обозначение $\bar{\pi}^{\mathrm{p}}$ закреплено за продолжением представления $\pi$ на пополнение Шлихтинга, мы используем в этом разделе обозначение $\pi^{\text {op }}$ для представления, сопряженного к $\pi_{0}$.

Это ситуация примера 28 в разделе 4 , когда $G=\operatorname{PGL}(2, \mathbb{Z}[1 / p]), p$ простое, $\Gamma$ - модулярная группа, $\mathscr{X}=\mathbb{H}$, а $\pi_{\text {Kоoр }}$ - представление Купмана на $L^{2}\left(\mathbb{H}, \nu_{0}\right)$, соответствующее действию группы $\operatorname{PSL}(2, \mathbb{R})$ преобразованиями Мёбиуса на верхней полуплоскости. Используя технику квантизации Березина [3], независимо отмеченную в [3] (см. также [36]), получаем

$$
\pi_{\text {Koop }}=\pi_{n} \otimes \pi_{n}^{\text {op }}, \quad n \geqslant 1,
$$

где $\pi_{n}$ - любое представление из дискретной серии группы $\operatorname{PSL}(2, \mathbb{R})$. Мы используем это в качестве мотивации для непосредственного изучения представлений вида $\pi_{0} \otimes \pi_{0}^{\mathrm{op}}$, где $\pi_{0}$ такое же, как в предыдущем разделе.

Прежде чем приступить к этому анализу, отметим одно дополнительное свойство, присущее всем представлениям $\bar{\pi}_{0}^{\mathrm{p}}, \bar{\pi}^{\mathrm{p}}$, построенным в разделах 5 и 6 . Мы докажем, что эти представления находятся во взаимно однозначном соответствии со вполне положительными отображениями $\Phi-$ с *-представлениями системы операторов, введенными в определении 14.

Введем следующие обозначения. Если $g \in G$, положим $L_{g} \in C^{*}(G)$ равным свертке с $g$. Если $f$ - функция из $C(\bar{G})$, обозначим через $L_{f} \in C^{*}(\bar{G})$ оператор свертки с $f$. Такое представление играет роль "операторнозначного собственного вектора" для алгебры Гекке.

Действительно, мы докажем, в частности, следующее свойство отображения $\Phi$. Если $\sigma_{1}, \sigma_{2} \in G$ и

$$
\left[\Gamma \sigma_{1} \Gamma\right]\left[\Gamma \sigma_{2}\right]=\sum_{j}\left[\Gamma \theta_{j}\right]
$$


TO

$$
\Phi\left(L_{\chi_{\left[\Gamma \sigma_{1} \Gamma\right]}}\right) \Phi\left(L_{\chi_{\left[\Gamma \sigma_{2}\right]}}\right)=\sum_{j} \Phi\left(L_{\chi_{\left[\Gamma \theta_{j}\right]}}\right) .
$$

Если бы $\Phi$ принимало скалярные значения, то данное свойство было бы в точности тем свойством, которым обладало бы собственное значение для действия алгебры Гекке на $\ell^{2}(\Gamma \backslash G)$.

Пусть $\mathscr{A}_{0}(G, \bar{G}) \subseteq \mathscr{A}(G, \bar{G})$ - всюду плотная подалгебра, порожденная операторами свертки с элементами из $G$ и операторами свертки с характеристическими функциями смежных классов подгрупп из $\mathscr{S}$. В этом разделе мы обозначаем через $\mathscr{P}_{\pi_{0}, L}$ введенную в формуле (48) проекцию, соответствующую $\Gamma_{0}=\Gamma$.

Теорема 37. Пусть $\pi_{0}-$ представление группы $G$ из определения 1. Onределим линейное отображение $\Phi: \mathscr{A}_{0}(G, \bar{G}) \rightarrow B(L)$ следующим образом. Пусть $g \in G, \Gamma_{0} \in \mathscr{S}$, и пусть $g \bar{\Gamma}_{0}-$ соответствующий смежный класс. Положим

$$
\begin{gathered}
\Phi\left(L_{\chi_{g \bar{\Gamma}_{0}}}\right)=\sum_{\theta \in g \Gamma_{0}} P_{L} \pi_{0}(\theta) P_{L}, \\
\Phi\left(L_{g}\right)=P_{L} \pi\left(L_{g}\right) P_{L} .
\end{gathered}
$$

Тогда Ф обладает следующими свойствами.

(i) Представление $\left.\Phi\right|_{\mathscr{O}(K, G)}$ является *-представлением введенной в определении 8 системы операторов $\mathscr{O}(K, G)$, принимающим значения в $B(L)$.

(ii) Рассмотрим векторное пространство $L(K, \bar{G}) \subseteq \mathscr{O}(K, G)$, введенное в определении 8. Тогда $\left.\Phi\right|_{L(K, \bar{G})}$ принимает значения в $\left.B(L) \mathscr{P}_{\pi_{0}, L} u\right|_{\mathscr{H}_{0}(K, \bar{G})}$ является представлением *-алгебр алгебры $\mathscr{H}_{0}(K, \bar{G})$ в $\mathscr{P}_{\pi_{0}, L} B(L) \mathscr{P}_{\pi_{0}, L}$. Следовательно, $\left.\Phi\right|_{\mathscr{O}(K, G)}$ принимает значения в $B(L) \mathscr{P}_{\pi_{0}, L} B(L)$.

(iii) Отображение $\Phi$ вполне положительно на $\mathscr{A}_{0}(G, \bar{G})$, т.е. оно отображает положительные элементы вида $X^{*} X, X \in \mathscr{A}_{0}(G, \bar{G})$, в положительные элементы.

ДоказАТЕльство. Утверждение (i) следует из формулы (49). Формула (18) из леммы 9 в сочетании с леммой 41 также дает доказательство утверждения (i).

Утверждение (ii) следует из утверждения (i).

Докажем утверждение (iii). Используется тот факт, что свойство (i) доказывает положительность отображения $\Phi$ на положительных элементах $X^{*} X$, где $X \in \mathbb{C}\left(\chi_{\sigma K} \mid \sigma \in G\right)$. Для подгрупп $\Gamma_{0} \in \mathscr{S}$ мы рассуждаем следующим образом. Положим $K_{0}$ равным замыканию в $\bar{G}$ подгруппы $\Gamma_{0}$. Сначала используем лемму 38 , чтобы установить положительность отображения $\Phi_{\Gamma_{0}}\left(X_{0}^{*} X_{0}\right)$ для $X_{0}$ из $\mathbb{C}\left(\chi_{\sigma K_{0}} \mid \sigma \in G\right)$. Из формулы сведения (61) следует, что $\Phi\left(X_{0}^{*} X_{0}\right)$ также положительно. Теорема 37 доказана.

Данную теорему легко обобщить, заменяя $Г$ на подгруппу $\Gamma_{0} \in \mathscr{S}$. В этом случае имеется очевидное соотношение между соответствующими представлениями систем операторов (мы используем определения и обозначения из теоремы 37). 
Лемма 38. Пусть $\Gamma_{0}$ - любая подгруппа из $\mathscr{S}$, и пусть $L^{\Gamma_{0}}$ определено в теореме 33. Тогда, используя $L^{\Gamma_{0}}$ вместо L, можно повторить описанное выше построение для $\Gamma_{0}$ вместо группы Г. Соответствующее вполне положительное отображение $\Phi_{\Gamma_{0}}$, построенное в теореме 37 , будет иметь те же свойства, что и $\Phi$, с заменой $\Gamma, K$ на $\Gamma_{0}, \bar{\Gamma}_{0}$.

Вложим $L$ в $L_{0}^{\Gamma}=\bigoplus_{i} \pi\left(s_{i}\right) L \subseteq H_{0}$, отображая $l \in L$ в вектор $l \oplus 0 \oplus 0 \oplus \cdots$. (Заметим, что это вложение отлично от диагоналъного вложения пространства $L$ в $L_{0}^{\Gamma}$, которое мы использовали в теореме 33 .) Обозначим через $\widetilde{P}_{L}$ проекцию из $L^{\Gamma_{0}}$ на L. Тогда

$$
\Phi=\widetilde{P}_{L} \Phi_{\Gamma_{0}} \widetilde{P}_{L}
$$

ДоказАТЕЛьство. Это легко следует из формулы (60).

ЗАмечание 39. Операторы $\Phi\left(\chi_{\sigma K}\right) \in B(L), \sigma \in G$, не являются изометриями, так как $\Phi$ не является представлением *-алгебр. Однако, как мы сейчас покажем, операторы $\Phi\left(\chi_{\sigma K}\right)$ являются произведениями проекции и изометрии.

Действительно, для $\sigma \in G$ частичная изометрия $L\left(\chi_{\sigma K}\right)$ имеет в качестве исходного пространства проекцию $L\left(\chi_{\sigma K \sigma^{-1}}\right)$, а в качестве образа $L\left(\chi_{K}\right)$.

Рассмотрим пространства $L^{\Gamma_{0}}, \Gamma_{0} \in \mathscr{S}$, введенные в определении 19. Эти пространства были определены там только в случае, когда $\Gamma_{0}-$ подгруппа группы Г. Определим $L^{\sigma \Gamma \sigma^{-1}} \subseteq L^{\Gamma_{\sigma}}$ формулой

$$
L^{\sigma \Gamma \sigma^{-1}}=\pi_{0}(\sigma) L
$$

Тогда

$$
\bar{\pi}^{\mathrm{p}}\left(L\left(\chi_{\sigma K}\right)\right)=P_{L^{\sigma \Gamma \sigma^{-1}}} \bar{\pi}_{0}^{\mathrm{p}}\left(L\left(\chi_{\sigma K}\right)\right) P_{L} .
$$

С другой стороны, используя скрещенное вложение пространства $L$ в $L^{\Gamma}$ из леммы 38 , получаем, что

$$
\Phi\left(\chi_{\sigma K}\right)=\widetilde{P}_{L} \bar{\pi}_{0}^{\mathrm{p}}\left(L\left(\chi_{\sigma K}\right)\right) P_{L} .
$$

Здесь проекция $P_{L}$ соответствует стандартному вложению пространства $L$ в $L^{\Gamma_{\sigma}}$, описанному в определении 19 , а $\widetilde{P}_{L}-$ проекция из леммы 38 .

Вполне положительные отображения, определенные в теореме 37, являются строительными блоками операторов Гекке. Следующий результат показывает, что представление из теоремы 37 кодирует все свойства представления $\bar{\pi}_{0}^{\mathrm{p}}$. Поэтому, зная $\Phi$, можно восстановить представление $\bar{\pi}_{0}^{\mathrm{p}}$, а значит, и представление $\pi$.

ПреДЛОЖЕНИЕ 40. В контексте теоремы 37 формула для операторов Гекке, предложенная в теореме 33 , имеет следующий вид. Зафиксируем $\Gamma_{0} \in \mathscr{S}$ u выберем разложение на смежные классы $\Gamma=\bigcup s_{i} \Gamma_{0}$. Тогда

$$
\left[\Gamma_{0}:\left(\Gamma_{0}\right)_{\sigma}\right] P_{H_{0}^{\Gamma_{0}}} \bar{\pi}_{0}^{\mathrm{p}}(\sigma) P_{H_{0}^{\Gamma_{0}}}=\sum_{i, j} \Phi\left(\chi_{\bar{s}_{i}^{-1} \Gamma_{0} \sigma \Gamma_{0} s_{j}}\right) \otimes e_{\Gamma_{0} s_{i}, \Gamma_{0} s_{j}}, \quad \sigma \in G .
$$

Следовательно, имеется взаимно однозначное соответствие между представлениями $\pi_{0}$ из определения 1 и вполне положительными отображениями $\Phi$ со свойствами (i)-(iii) из теоремы 37. 
ДоказАТЕльство. Формула (62) есть не что иное, как формула (55) из теоремы 34, переписанная в новом контексте с помощью формулы (60) из теоремы 37.

Предположим, что имеют место свойства (i)-(iii) из теоремы 37. Докажем, что операторы в формуле (62) определяют представление алгебры Гекке двойных смежных классов для всех $\Gamma_{0} \in \mathscr{S}$.

Проверка мультипликативности операторов Гекке, заданных формулой (62), сводится к проверке тождества вида

$$
\sum_{\gamma_{0} \in \Gamma_{0}} P_{L^{\Gamma_{0}}} \pi_{0}\left(\sigma_{1} \gamma_{0}\right) P_{L^{\Gamma_{0}}}\left(\pi_{0}\left(\gamma_{0}^{-1} \sigma_{2}\right)\right) P_{L^{\Gamma_{0}}}=P_{L^{\Gamma_{0}}}\left(\pi_{0}\left(\sigma_{1} \sigma_{2}\right)\right) P_{L^{\Gamma_{0}}}
$$

Причина, по которой выполняется это равенство, состоит в том, что $P_{L^{\Gamma_{0}}}-$ проекция на $\Gamma_{0}$-блуждающее порождающее подпространство пространства $H$ (см. также приводимое далее доказательство леммы 9). Таким образом, единственное тождество, в силу которого представление в формуле (62) является представлением алгебры Гекке, - это тождество

$$
\sum_{\gamma_{0} \in \Gamma_{0}} \pi\left(\gamma_{0}\right) P_{L^{\Gamma_{0}}} \pi\left(\gamma_{0}^{-1}\right)=\operatorname{Id}_{H_{0}}
$$

В силу разложения $P_{L^{\Gamma_{0}}}=\sum_{i} \pi\left(s_{i}\right) P_{L} \pi\left(s_{i}\right)$, это вытекает из тождества

$$
\sum_{\gamma \in \Gamma} \pi(\gamma) P_{L} \pi\left(\gamma^{-1}\right)=\operatorname{Id}_{H_{0}}
$$

Но это есть в точности тождество, доказывающее свойство мультипликативности (ii) в теореме 37.

Таким образом, если мы знаем, что $\Phi$ обладает свойством мультипликативности (ii) из теоремы 37 , то мы автоматически получаем, что вполне положительные отображения $\Phi_{\Gamma_{0}}$ обладают соответствующим свойством мультипликативности из п. (ii) на соответствующих системах операторов $\mathscr{O}\left(\bar{\Gamma}_{0}, \bar{G}\right)$ для $\Gamma_{0} \in \mathscr{S}$. Пусть $\mathscr{P}_{\Gamma_{0}, L}-$ проекция, введенная в (48).

Поскольку системы операторов содержат соответствующие алгебры Гекке $\mathscr{H}_{0}\left(\bar{\Gamma}_{0}, \bar{G}\right)$, то представление в формуле $(62)$ является представлением $*$-алгебр индуктивного предела всех вышеупомянутых алгебр Гекке в индуктивном пределе пространств $\mathscr{P}_{\Gamma_{0}, L} B\left(L^{\Gamma_{0}}\right) \mathscr{P}_{\Gamma_{0}, L}$. Но этот индуктивный предел есть в точности пространство ограниченных операторов, действующих на гильбертовом пространстве $\bar{H}_{0}^{\mathrm{p}}$. Так как наряду с алгебрами Гекке у нас также есть представление пространств смежных классов, получаем, что мы восстановили унитарное представление $\bar{\pi}_{0}^{\mathrm{p}}$ алгебры $C^{*}(\bar{G})$. Отсюда можно восстановить $\pi_{0}$ в силу теоремы 37. Предложение доказано.

В обсуждаемых ниже утверждениях (леммы 9, 41 и теорема 10) мы напоминаем результаты из [38], см. также [37], [40] (мы адаптируем формулировки этих результатов к рамкам настоящей работы). Лемма 9 дополняет утверждение теоремы 37. Мы доказываем, что вполне положительные отображения из теоремы 37 обладают естественным поднятием на алгебру $\mathscr{L}(G) \otimes B(L)$. Это поднятие послужило важным инструментом при доказательстве в работе [37] 
оценок спектра операторов Гекке в существенной норме. В частности, мы даем другую интерпретацию свойства (ii) из теоремы 37.

ДокАЗАТЕЛЬство ЛЕммы 9 (доказательство можно также найти в [38; предложение 2.2 и лемма 3.1]). Основной шаг вывода свойства мультипликативности в формуле (18) состоит в следующем: отождествляя коэффициенты элементов $\rho(g), g \in G$, в обеих частях равенства, мы сводим доказательство свойства мультипликативности к доказательству следующего равенства (используемого также в доказательстве предложения 40):

$$
\begin{gathered}
\sum_{\gamma \in \Gamma} P_{L} \pi_{0}\left(\sigma_{1} \gamma\right) P_{L} \pi_{0}\left(\gamma^{-1} \sigma_{2}\right) P_{L}=\sum_{\gamma \in \Gamma} P_{L} \pi_{0}\left(\sigma_{1}\right) \pi(\gamma) P_{L} \pi\left(\gamma^{-1}\right) \pi_{0}\left(\sigma_{2}\right) P_{L} \\
=P_{L} \pi_{0}\left(\sigma_{1}\right) \pi_{0}\left(\sigma_{2}\right) P_{L}=P_{L} \pi_{0}\left(\sigma_{1} \sigma_{2}\right) P_{L}, \quad \sigma_{1}, \sigma_{2} \in G .
\end{gathered}
$$

Операторы $\widetilde{\Phi}_{\pi_{0}, L}$, определенные в лемме 9 , допускают интерпретацию, аналогичную приведенной в замечании 39 . Более того, в силу сделанного в теореме 33 предположения о сходимости, для сумм вида $\sum_{\theta \in C} P_{L} \pi_{0}(\theta) P_{L}$ со смежными классами $C$ в $\bar{G}$ операторы $\widetilde{\Phi}_{\pi_{0}, L}(C)$ являются поднятиями операторов $\Phi(C)$, определенных в теореме 37.

Это доказывается в следующем утверждении, которое является несложным следствием теоремы 37 и леммы 9.

Лемма 41. Пусть $\varepsilon-$ неограниченный характер на $\ell^{1}(G) \subseteq \mathscr{L}(G)$, который ставит в соответствие элементу $x \in \ell^{1}(G)$ сумму его коэффициентов. Продолжим $\varepsilon$ до неограниченного характера $\widetilde{\varepsilon}=\varepsilon \otimes \operatorname{Id}_{B(L)}$ :

$$
\widetilde{\varepsilon}: \ell^{1}(G) \otimes B(L) \subseteq \mathscr{R}(G) \otimes B(L) \rightarrow B(L) .
$$

Из предположения о сходимости (v) в определении 1 следует, что образ *-представления $\widetilde{\Phi}_{\pi_{0}, L}$, построенного в лемме 9 , содержится в области определения характера $\widetilde{\varepsilon}$. Поэтому для $\Phi$, определенного в теореме 37 , имеет место следующая коммутативная диаграмма:

$$
\left.\widetilde{\varepsilon} \circ \widetilde{\Phi}_{\pi_{0}, L}\right|_{\mathscr{O}(K, \bar{G})}=\left.\Phi\right|_{\mathscr{O}(K, \bar{G})} .
$$

ДокАзАтельство. Утверждение сразу следует из формул для $\Phi$ и $\widetilde{\Phi}_{\pi_{0}, L}$ в теореме 37 и лемме 9 соответственно.

Операторы $\widetilde{\Phi}_{\pi_{0}, L}$ используются для построения унитарно эквивалентного представления (см. формулу (19) для операторов Гекке, ассоциированных с унитарным диагональным представлением $\pi_{0} \otimes \pi_{0}^{\text {op }}$ группы $G$, где $\pi_{0}$ - такое, как в определении 1). Это было впервые доказано (в случае размерности Мюррея-фон Неймана, равной единице) в [37; теорема 22] (см. также более краткое изложение в [40]) и затем обобщено на произвольную размерность в [38; теорема 3.2]. Для удобства читателя (так как мы объясняем пример представления алгебры Гекке, ассоциированной с унитарным представлением $\pi_{0} \otimes \pi_{0}^{\text {op }}$ группы $G$ ) мы напоминаем формулировку теоремы 3.2 из [38]. В теореме 12 мы приводим другое доказательство того, что формула (19) дает представление алгебры Гекке двойных смежных классов подгруппы $\Gamma$ в $G$. 
Мы используем отождествления, доказанные в примере 30 , и операторы $\widetilde{\Phi}_{\pi_{0}, L}$, введенные в лемме 9, чтобы явно описать операторы Гекке на Г-инвариантных векторах, ассоциированные с унитарным диагональным представлением $\pi_{0} \otimes \pi_{0}^{\text {op }}$ группы $G$. В этом случае легче работать с гильбертовыми пространствами Г-инвариантных векторов, так как эти пространства можно канонически отождествить с $L^{2}$-пространствами алгебры фон Неймана операторов, коммутирующих с образом представления группы $Г$.

В [37], используя методы квантизации Березина [3] или же результаты из [42], мы доказали, что вышеописанная модель для операторов Гекке, действующих на Г-инвариантных векторах для $\pi_{0} \otimes \pi_{0}^{\mathrm{op}}$, унитарно эквивалентна представлению операторов Гекке ассоциированной алгебры фон Неймана форм Маасса.

Содержание теоремы 10 заключается в явной модели в терминах операторных алгебр для предыдущего представления, ассоциированного с $\pi_{0} \otimes \pi_{0}^{\text {op }}$.

Мы приводим прямое доказательство в частном случае $\operatorname{dim}_{\left\{\pi_{0}(\Gamma)\right\}^{\prime \prime}} H=1$ (теорема 12) того факта, что операторы Гекке, введенные формулой (19) в теореме 10, определяют мультипликативное представление алгебры Гекке двойных смежных классов группы $\Gamma$ в $G$. Это доказательство показывает, что представление алгебры Гекке, определяемое операторами Гекке для $\pi_{0} \otimes \pi_{0}^{\text {op }}$, получается из канонического представления алгебры Гекке, которое далее берется в композиции с факторотображением. Для простоты изложения предполагаем далее, что группы $Г$ и $G$ имеют бесконечные нетривиальные классы сопряженности и, значит, ассоциированные алгебры фон Неймана имеют единственные следы.

ЗАмЕчание 42. Предположим, что $\operatorname{dim}_{\left\{\pi_{0}(г)\right\}^{\prime \prime}} H=1$. В постановке теоремы 22 в разделе 3 мы берем $L=L_{0}=\mathbb{C} \xi$, где $\xi \in H_{0}$ - циклический следовой вектор для $\left.\pi\right|_{\Gamma}$. Построение в лемме 9 дает линейное отображение $\widetilde{\Phi}_{\pi_{0}, L_{0}}$, которое мы теперь обозначаем через $t$ :

$$
t: \mathscr{H}_{\text {red }}(\Gamma, G) \rightarrow \mathscr{R}(G) \otimes B\left(L_{0}\right) \cong \mathscr{R}(G) .
$$

Так как $L_{0}$ одномерно, то с помощью вектора $\xi$ отождествляя $L_{0}=\mathbb{C} \xi \mathrm{c} \mathbb{C}$, получаем, что можно заменить $P_{L_{0}} \pi_{0}(\theta) P_{L_{0}}$ на

$$
\operatorname{Tr}\left(P_{L_{0}} \pi_{0}(\theta) P_{L_{0}}\right)=\left\langle\pi_{0}(\theta) \xi, \xi\right\rangle, \quad \theta \in G .
$$

Для смежного класса $C$ подгруппы из $\mathscr{S}$ формула для $\widetilde{\Phi}_{\pi_{0}, L_{0}}\left(\chi_{C}\right)$ из леммы 9 теперь имеет вид

$$
t\left(\chi_{C}\right)=\sum_{\theta \in C}\left\langle\pi_{0}(\theta) \xi, \xi\right\rangle \rho(\theta) .
$$

Рассмотрим композицию отображения $t$ с каноническим антиизоморфизмом между $\mathscr{L}(G)$ и $\mathscr{R}(G)$ (для простоты обозначим ее снова через $t$ ). Таким образом, $t$ - линейное отображение из $C^{*}(G)$ со значениями в $\mathscr{L}(G)$. Обозначим через $\lambda_{g}$ левые свертки элементами $g \in G$. Тогда $t$ задается формулой

$$
t\left(\chi_{C}\right)=\sum_{\theta \in C} \overline{\left\langle\pi_{0}(\theta) \xi, \xi\right\rangle} \lambda(\theta)
$$


В силу леммы 9 отображение $t$ является $*$-сохраняющим мультипликативным представлением системы операторов $\mathscr{O}_{\Gamma, G}=\mathscr{O}(K, \bar{G})$, введенной в определении 8:

$$
\mathscr{O}_{\Gamma, G}=\left[\operatorname{Sp}\left\{\chi_{\sigma_{1} K} \mid \sigma_{1} \in G\right\}\right]\left[\operatorname{Sp}\left\{\chi_{\sigma_{2} K} \mid \sigma_{2} \in G\right\}\right]^{*} .
$$

Воспользуемся соответствием между характеристическими функциями $\chi_{\sigma_{1} K}$, $\chi_{K \sigma_{2}}, \chi_{\sigma_{1} K \sigma_{2}}$ для $\sigma_{1}, \sigma_{2} \in G$ и смежными классами $\sigma_{1} \Gamma, \Gamma \sigma_{2}, \sigma_{1} \Gamma \sigma_{2}$ в $G$. Тогда *-сохраняющее свойство мультипликативности для $\left.t\right|_{\mathscr{O}_{\Gamma, G}}$ примет вид

$$
t\left(\sigma_{1} \Gamma\right) t\left(\sigma_{2}^{-1} \Gamma\right)^{*}=t\left(\sigma_{1} \Gamma\right) t\left(\Gamma \sigma_{2}\right)=t\left(\sigma_{1} \Gamma \sigma_{2}\right), \quad \sigma_{1}, \sigma_{2} \in G .
$$

ЗАмЕчАНИЕ 43. На практике трудно найти циклический следовой вектор $\xi$ фигурирующий выше. Поэтому предпочтительнее использовать построение из теоремы 33 раздела 5 . Таким образом, $\pi_{0}$ получается из большего представления $\pi$ группы $G$ в унитарную группу гильбертова пространства $H$ ограничением на пространство $H_{0} \subseteq H$, которое инвариантно относительно $\pi(G)$. В этом случае мы используем выбор Г-блуждающего порождающего подпространства $L$ для $\left.\pi\right|_{\Gamma}$. В аналитической дискретной серии унитарных представлений $\pi_{n}, n \geqslant 1$, группы $\operatorname{PSL}(2, \mathbb{R})$ такой выбор является почти каноническим, так как он состоит в выборе фундаментальной области для действия группы Г на $\mathbb{H}$.

Чтобы непосредственно получить из $\widetilde{\Phi}_{\pi_{0}, L}$ представление $t$, можно действовать напрямую следующим образом [38]. Рассмотрим условные математические ожидания

$$
E_{\mathscr{R}(G) \otimes \mathbb{C} \operatorname{Id}_{B(L)}}^{\mathscr{R}(G) \otimes B(L)}, \quad E_{\mathscr{R}(\Gamma) \otimes \mathbb{C} \operatorname{Id}_{B(L)}}^{\mathscr{R}(\Gamma) \otimes B(L)}
$$

соответственно из $\mathscr{B}=\mathscr{R}(G) \otimes B(L)$ и $\mathscr{A}=\mathscr{R}(\Gamma) \otimes B(L)$ на алгебры $\mathscr{R}(G) \otimes$ $\mathbb{C} \operatorname{Id}_{B(L)}$ и $\mathscr{R}(\Gamma) \otimes \mathbb{C} \operatorname{Id}_{B(L)}$. Условные математические ожидания просто вычисляются взятием операторного следа на тензорном множителе, соответствующем $B(L)$.

Для смежного класса $C$, указанного в замечании 42, имеем

$$
\tilde{t}\left(\chi_{C}\right)=E_{\mathscr{R}(\Gamma) \otimes \operatorname{Id}_{B(L)}}^{\mathscr{R}(G) \otimes B(L)}\left(\widetilde{\Phi}_{\pi_{0}, L}\left(\chi_{C}\right)\right)=\sum_{\theta \in C} \operatorname{Tr}\left(P_{L} \pi_{0}(\theta)\right) \rho(\theta) .
$$

Мы используем формулу (17) для проекции $P_{0}$ и определяем

$$
\xi_{0}=E_{\mathscr{R}(\Gamma) \otimes B(L)}^{\mathscr{R}(\Gamma) \operatorname{Id}_{B(L)}}\left(P_{0}\right)=\sum_{\gamma \in \Gamma} \operatorname{Tr}\left(P_{L} \pi_{0}(\gamma)\right) \rho(\gamma) .
$$

Так как $\operatorname{dim}_{\left\{\pi_{0}(\Gamma)\right\}^{\prime \prime}} H_{0}=1$, а $P_{0}-$ проекция из $\mathscr{A} \subseteq \mathscr{B}$ со следом 1 , получаем, что $\xi_{0}$ имеет нулевое ядро. Более того (см. [38; предложение 3.3]), отображение условного математического ожидания, подправленное обратным квадратного корня из $\xi_{0}$, является изоморфизмом алгебр фон Неймана, будучи ограниченным на $P_{0} \mathscr{B} P_{0}$. Таким образом,

$$
\widetilde{E}=\left.\left(\xi_{0}\right)^{-1 / 2} E_{\mathscr{R}(\Gamma) \otimes \operatorname{Id}_{B(L)}}^{\mathscr{R}(G) \otimes B(L)}\right|_{P_{0} \mathscr{B} P_{0}}\left(\xi_{0}\right)^{-1 / 2}
$$

является изоморфизмом алгебр фон Неймана из $P_{0} \mathscr{B} P_{0}$ на $\mathscr{R}(G)$. Определим

$$
t\left(\chi_{C}\right)=\widetilde{E}(\tilde{t}(C)) .
$$


Тогда $\left.t\right|_{\mathscr{O}_{\Gamma, G}}-$ изоморфизм из $\mathscr{H}(\Gamma, G)$ в $\mathscr{R}(G)$ (см. [38; доказательство леммы 3.3]). Объединяя формулы (64) и (65), получаем следующую альтернативную формулу для представления $t$ из замечания 42 :

$$
t\left(\chi_{C}\right)=\left(\xi_{0}\right)^{-1 / 2}\left[\sum_{\theta \in C} \operatorname{Tr}\left(P_{L} \pi_{0}(\theta)\right) \rho(\theta)\right]\left(\xi_{0}\right)^{-1 / 2} .
$$

ДокАЗАТЕЛЬСтво тЕоремы 12. Зафиксируем $\sigma \in G$. Тогда двойной смежный класс Г $\sigma \Gamma$ допускает следующее разложение: $Г \sigma \Gamma=\bigcup \Gamma \sigma s_{i}=\bigcup r_{j} \sigma \Gamma$. Отсюда вытекает, что

$$
\begin{aligned}
\chi_{K}\left(L\left(\chi_{K \sigma K}\right) \otimes L\left(\chi_{K \sigma K}\right)^{\mathrm{op}}\right) \chi_{K}=\sum_{i, j}\left[L\left(\chi_{K \sigma s_{i}}\right) \otimes L\left(\chi_{K \sigma s_{j}}\right)^{\mathrm{op}}\right] \chi_{s_{i} \sigma^{-1} K \sigma s_{j}^{-1} \cap K} \\
\quad=\sum_{a, b} \chi_{r_{a} \sigma K \sigma^{-1} r_{b} \cap K}\left[L\left(\chi_{r_{a} \sigma K}\right) \otimes L\left(\chi_{r_{b} \sigma K}\right)^{\mathrm{op}}\right] \\
=\sum_{i, j, a, b} \chi_{r_{a} \sigma K \sigma^{-1} r_{b} \cap K}\left[L\left(\chi_{r_{a} \sigma K_{\sigma-1} s_{i}}\right) \otimes L\left(\chi_{r_{b} \sigma K_{\sigma^{-1}} s_{j}}\right)^{\mathrm{op}}\right] \chi_{s_{i} \sigma^{-1} K \sigma s_{j} \cap K} .
\end{aligned}
$$

Здесь $K_{\sigma^{-1}}$ - замыкание в $K$ подгруппы $\Gamma_{\sigma^{-1}}=\sigma^{-1} \Gamma \sigma \cap \Gamma$.

С использованием равенства (66) немедленно доказывается (см., например, вычисления в $[37 ; \S 5]$ или [40]), что линейное отображение в данном утверждении мультипликативно. Это завершает доказательство части (i).

Представление $t$ системы операторов $\mathscr{O}_{\Gamma, G}=\mathscr{O}(K, \bar{G})$ продолжается очевидным образом до представления $\tilde{t}$ системы операторов

$$
\left(L^{\infty}(\bar{G}, \mu) \operatorname{Sp}\left\{L\left(\chi_{\sigma_{1} K}\right) \mid \sigma_{1} \in G\right\}\right)\left(L^{\infty}(\bar{G}, \mu) \operatorname{Sp}\left\{L\left(\chi_{\sigma_{2} K}\right) \mid \sigma_{2} \in G\right\}\right)^{*} .
$$

Тогда $\tilde{t}$ продолжается до “двойного” представления $t_{2}$ системы операторов, содержащейся в алгебре $C^{*}\left(\left(G \times G^{\text {op }}\right) \rtimes L^{\infty}(\bar{G}), \mu\right)$, которая содержит образ алгебры Гекке, построенной в п. (i). Это завершает доказательство части (ii).

Важное наблюдение для доказательства части (iii) состоит в том, что все операции, вовлеченные в умножении двух элементов вида $\chi_{K}\left(L\left(\chi_{K \sigma_{1} K}\right) \otimes\right.$ $\left.L\left(\chi_{K \sigma_{1} K}\right)^{\mathrm{op}}\right) \chi_{K}$, остаются внутри области определения представления $t_{2}$ (см. первое равенство в (66)). Действительно, эти операции включают только свертки вида

$$
L\left(\chi_{\sigma_{1} K}\right) L\left(\chi_{K \sigma_{2}}\right), \quad \sigma_{1}, \sigma_{2} \in G,
$$

или их противоположные.

Следовательно, композиция $t_{2}$ с отображением $\overline{\mathscr{D} \Phi}$, введенным в утверждении (i) теоремы, дает представление алгебры Гекке. Теорема 12 доказана.

\section{Список литературы}

[1] A. N. Andrianov, Quadratic forms and Hecke operators, Grundlehren Math. Wiss., 286, Springer-Verlag, Berlin, 1987, xii+374 pp.

[2] M. B. Bekka, R. Curtis, P. de la Harpe, "Familles de graphes expanseurs et paires de Hecke", C. R. Math. Acad. Sci. Paris, 335:5 (2002), 463-468. 
[3] Ф.А. Березин, "Квантование", Изв. АН СССР. Сер. матем., 38:5 (1974), 1116-1175; англ. пер.: F. А. Berezin, "Quantization", Math. USSR-Izv., 8:5 (1974), 1109-1165.

[4] M. W. Binder, "Induced factor representations of discrete groups and their types", J. Funct. Anal., 115:2 (1993), 294-312.

[5] F. Boca, V. Nitică, "Combinatorial properties of groups and simple $C^{*}$-algebras with a unique trace", J. Operator Theory, 20:1 (1988), 183-196.

[6] A. Borel, "Admissible representations of a semi-simple group over a local field with vectors fixed under an Iwahori subgroup", Invent. Math., 35 (1976), 233-259.

[7] J.-B. Bost, A. Connes, "Hecke algebras, type III factors and phase transitions with spontaneous symmetry breaking in number theory", Selecta Math. (N. S.), 1:3 (1995), 411-457.

[8] L. Bowen, "Invariant random subgroups of the free group", Groups Geom. Dyn., 9:3 (2015), 891-916; 2014 (v1 - 2012), 17 pp., arXiv: 1204.5939.

[9] L. Bowen, R. Grigorchuk, R. Kravchenko, Characteristic random subgroups of geometric groups and free abelian groups of infinite rank, 2015 (v1 - 2014), 32 pp., arXiv: 1402.3705.

[10] N.P. Brown, N. Ozawa, $C^{*}$-algebras and finite-dimensional approximations, Grad. Texts in Math., 88, Amer. Math. Soc., Providence, RI, 2008, xvi+509 pp.

[11] M. Burger, J.-S. Li, P. Sarnak, "Ramanujan duals and automorphic spectrum", Bull. Amer. Math. Soc. (N. S.), 26:2 (1992), 253-257.

[12] P. Cartier, "Representations of p-adic groups: a survey", Automorphic forms, representations and L-functions, Part 1 (Oregon State Univ., Corvallis, OR, 1977), Proc. Sympos. Pure Math., 33, Amer. Math. Soc., Providence, RI, 1979, 111-155.

[13] W. Casselman, "Harmonic analysis of the Schwartz space of $\Gamma \backslash \mathrm{SL}_{2}(\mathbb{R})$ ", Contributions to automorphic forms, geometry, and number theory, Johns Hopkins Univ. Press, Baltimore, MD, 2004, 163-192.

[14] R. Curtis, "Hecke algebras associated with induced representations", C. R. Math. Acad. Sci. Paris, 334:1 (2002), 31-35.

[15] P. Deligne, "La conjecture de Weil. I", Inst. Hautes Études Sci. Publ. Math., 43 (1974), 273-307.

[16] A. Dudko, K. Medynets, "Finite factor representations of Higman-Thompson groups", Groups Geom. Dyn., 8:2 (2014), 375-389.

[17] И. М. Гельфанд, М. И. Граев, И. И. Пятецкий-Шапиро, Обобщеннъе функиии, т. 6 : Теория представлений и автоморфные функиии, Наука, М., 1966, 512 с.; англ. пер.: I. M. Gel'fand, M. I. Graev, I. Pyatetskii-Shapiro, Representation theory and automorphic functions, W. B. Saunders Co., Philadelphia, PA-London-Toronto, ON, 1969, xvi+426 pp.

[18] И. М. Гельфанд, Обобщенные функиии, т. 4: Некоторые применения гармонического анализа. Оснащенные гильбертовы пространства, Наука, М., 1961, 472 с.; англ. пер.: I. M. Gel'fand, N. Ja. Vilenkin, Generalized functions, v. 4: Applications of harmonic analysis, Academic Press, New York-London, 1964, xiv+384 pp.

[19] F. M. Goodman, P. de la Harpe, V.F. R. Jones, Coxeter graphs and towers of algebras, Math. Sci. Res. Inst. Publ., 14, Springer-Verlag, New York, 1989, x+288 pp.

[20] U. Haagerup, T. Steenstrup, R. Szwarc, "Schur multipliers and spherical functions on homogeneous trees", Internat. J. Math., 21:10 (2010), 1337-1382.

[21] R.W. Hall, Hecke C*-algebras, Thesis (Ph. D.), Pennsylvania State Univ., 1999, $128 \mathrm{pp}$. 
[22] Harish-Chandra, "Plancherel formula for the $2 \times 2$ real unimodular group", Proc. Nat. Acad. Sci. U.S. A., 38 (1952), 337-342.

[23] E. Hecke, Lectures on Dirichlet series, modular functions and quadratic forms, Vandenhoeck \& Ruprecht, Göttingen, 1983, 98 pp.

[24] D. A. Hejhal, The Selberg trace formula for $\operatorname{PSL}(2, \mathbb{R})$, v. I, Lecture Notes in Math., 548, Springer-Verlag, Berlin-New York, 1976, vi+516 pp.

[25] V. F. R. Jones, "Index for subfactors", Invent. Math., 72:1 (1983), 1-25.

[26] S. Kaliszewski, B. L. Magnus, J. Quigg, "Hecke $C^{*}$-algebras and semi-direct products", Proc. Edinb. Math. Soc. (2), 52:1 (2009), 127-153.

[27] A.S. Kechris, "Unitary representations and modular actions", Теория представлений, динамические системы, комбинаторные и алгоритмические методы. XIII, Зап. науч. сем. ПОМИ, 326, ПОМИ, СПб., 2005, 97-144; J. Math. Sci. (N. Y.), 140:3 (2007), 398-425.

[28] A. Krieg, Hecke algebras, Mem. Amer. Math. Soc., 87, № 435, Amer. Math. Soc., Providence, RI, 1990, x+158 pp.

[29] M. Laca, N.S. Larsen, S. Neshveyev, "Phase transition in the Connes-Marcolli $\mathrm{GL}_{2}$-system", J. Noncommut. Geom., 1:4 (2007), 397-430.

[30] S. Lang, SL $2(\mathbb{R})$, Grad. Texts in Math., 105, Springer-Verlag, New York, 1985, xiv +428 pp.

[31] H. Maass, Lectures on modular functions of one complex variable, With notes by S. Lal, 2nd ed., Tata Inst. Fund. Res. Stud. Lectures on Math. and Phys., 29, Tata Inst. Fund. Res., Bombay, 1983, iii+262 pp.

[32] Yu. A. Neretin, "Plancherel formula for Berezin deformation of $L^{2}$ on Riemannian symmetric space", J. Funct. Anal., 189:2 (2002), 336-408.

[33] J. Peterson, A. Thom, "Character rigidity for special linear groups", J. Reine Angew. Math., 1-22, publ. online 11.03.2014.

[34] H. Petersson, "Über automorphe Formen mit Singularitäten im Diskontinuitätsgebiet", Math. Ann., 129 (1955), 370-390.

[35] G. Pisier, Introduction to operator space theory, London Math. Soc. Lecture Note Ser., 294, Cambridge Univ. Press, Cambridge, 2003, viii+478 pp.

[36] F. Rădulescu, The $\Gamma$-equivariant form of the Berezin quantization of the upper half plane, Mem. Amer. Math. Soc., 133, № 630, Amer. Math. Soc., Providence, RI, 1998, viii+70 pp.

[37] F. Rădulescu, Type $I I_{1}$ von Neumann representations for Hecke operators on Maass forms and Ramanujan-Petersson conjecture, 2013 (v1 - 2008), 43 pp., arXiv: 0802.3548 .

[38] F. Rădulescu, "Conditional expectations, traces, angles between spaces and representations of the Hecke algebras", Lib. Math. (N. S.), 33:2 (2013), 65-95.

[39] F. Rădulescu, The operator algebra content of the Ramanujan-Petersson problem, 2014 (v1 - 2013), 53 pp., arXiv: 1306.4232.

[40] F. Rădulescu, "Free group factors and Hecke operators", The varied landscape of operator theory, Theta Ser. Adv. Math., Theta, Bucharest, 2014, 241-257.

[41] F. Rădulescu, "On the countable measure-preserving relation induced on a homogeneous quotient by the action of a discrete group", Complex Anal. Oper. Theory, 9:7 (2015), 1633-1662.

[42] J. Repka, "Tensor products of holomorphic discrete series representations", Canad. J. Math., 31:4 (1979), 836-844. 
[43] J. Roe, "On the quasi-isometry invariance of $L^{2}$ Betti numbers", Duke Math. J., 59:3 (1989), 765-783.

[44] S. Sakai, C*-algebras and $\mathrm{W}^{*}$-algebras, reprint of 1st (1971) ed., Classics Math., Springer-Verlag, Berlin, 1998, xii+256 pp.

[45] P. J. Sally, Jr., J.A. Shalika, "Characters of the discrete series of representations of SL(2) over a local field", Proc. Nat. Acad. Sci. U.S.A., 61 (1968), 1231-1237.

[46] P. Sarnak, Some applications of modular forms, Cambridge Tracts in Math., 99, Cambridge Univ. Press, Cambridge, 1990, x+111 pp.

[47] P. Sarnak, "Notes on the generalized Ramanujan conjectures", Harmonic analysis, the trace formula, and Shimura varieties, Clay Math. Proc., 4, Amer. Math. Soc., Providence, RI, 2005, 659-685.

[48] G. Schlichting, "Operationen mit periodischen Stabilisatoren", Arch. Math. (Basel), 34:2 (1980), 97-99.

[49] F. Shahidi, " $L$-functions and representation theory of $p$-adic groups", p-adic methods and their applications, Oxford Sci. Publ., Oxford Univ. Press, New York, 1992, 91-112.

[50] M. Takesaki, Theory of operator algebras I, reprint of the 1st (1979) ed., Encyclopaedia Math. Sci., 124, Oper. Alg. Non-Commut. Geom., V, Springer-Verlag, Berlin, 2002, $\mathrm{xx}+415 \mathrm{pp}$.

[51] K. Tzanev, "Hecke $C^{*}$-algebras and amenability", J. Operator Theory, 50:1 (2003), 169-178.

[52] А. М. Вершик, "Несвободные действия счетных групп и их характеры", Теория представлений, динамические системы, комбинаторные методы. XVIII, Зап. науч. сем. ПОМИ, 378, ПОМИ, СПб., 2010, 5-16; англ. пер.: А. M. Vershik, "Nonfree actions of countable groups and their characters", J. Math. Sci. (N. Y.), 174:1 (2011), $1-6$.

[53] D. Zagier, Traces des opérateurs de Hecke. Théorie des nombres, Séminaire DelangePisot-Poitou, 17e année: 1975/76, fasc. 2, exp. № 23, Secrétariat Math., Paris, 1977, $12 \mathrm{pp}$.

Флорин Радулеску

(Florin Rădulescu)

Università degli Studi di Roma "Tor Vergata",

Roma, Italy;

Institute of Mathematics "Simion Stoilow"

of the Romanian Academy, Bucharest, Romania

E-mail: radulesc@mat.uniroma2.it
Поступила в редакцию 20.03.2015 